\title{
Stereoselective Synthesis of Isochromanones by an Asymmetric Ortholithiation Strategy: Synthetic Access to the Isochromanone Core of the Ajudazols
}

\author{
Sebastian Essig ${ }^{\dagger}$ and Dirk Menche ${ }^{{ }^{*}}$ \\ $\dagger$ Institut für Organische Chemie, Ruprecht-Karls Universität Heidelberg, Im Neuenheimer Feld 270, 69120 \\ Heidelberg, Germany, present address: MRC Laboratory of Molecular Biology, Francis Crick Avenue, CB2 \\ 0QH Cambridge, U.K. \\ $\$$ Kekulé-Institut für Organische Chemie und Biochemie, Universität Bonn, Gerhard-Domagk-Str. 1, 53121 \\ Bonn, Germany \\ dirk.menche@uni-bonn.de
}

\section{Table of Content}

I X-Ray crystal structure analysis

2

II References

9

III Copies of ${ }^{1} \mathrm{H}$ - and ${ }^{13} \mathrm{C}$-NMR spectra 


\section{X-Ray crystal structure analysis}

\section{X-ray crystal structure analysis of compound 54}

Description: colourless crystal (needle), dimensions $1.18 \times 0.15 \times 0.13 \mathrm{~mm}^{3}$, crystal system triclinic, space group $\mathrm{P} \overline{1}, \mathrm{Z}=2, \mathrm{a}=7.5558(10) \AA, \mathrm{b}=13.3993(17) \AA, \mathrm{c}=14.2195(18) \AA$, alpha $=83.276(3)$ $\mathrm{deg}$, beta $=80.259(3) \mathrm{deg}$, gamma $=78.211(3) \mathrm{deg}, \mathrm{V}=1383.8(3) \AA^{3}, \mathrm{rho}=1.158 \mathrm{~g} / \mathrm{cm}^{3}, \mathrm{~T}=200(2)$ $\mathrm{K}$, Theta $_{\max }=24.76 \mathrm{deg}$, radiation Mo Kalpha, lambda $=0.71073 \AA$, 0.3 deg omega-scans with CCD area detector, covering the asymmetric unit in reciprocal space with a mean redundancy of 4.1 and a completeness of $97.2 \%$ to a resolution of $0.84 \AA$, 18920 reflections measured, 4614 unique $(\mathrm{R}(\mathrm{int})=0.0381), 3709$ observed $(\mathrm{I}>2 \sigma(\mathrm{I})$ ), intensities were corrected for Lorentz and polarization effects, an empirical absorption correction was applied using SADABS, based on the Laue symmetry of the reciprocal space, $\mathrm{mu}=0.12 \mathrm{~mm}^{-1}, \mathrm{~T}_{\min }=0.87, \mathrm{~T}_{\max }=0.98$, structure solved by direct methods and refined against $\mathrm{F}^{2}$ with a Full-matrix least-squares algorithm using the SHELXTL (Version 2008/4) software package, ${ }^{1} 346$ parameters refined, hydrogen atoms were treated using appropriate riding models, goodness of fit 1.14 for observed reflections, final residual values $\mathrm{R} 1(\mathrm{~F})=0.055$, $w \mathrm{R}\left(\mathrm{F}^{2}\right)=$ 0.116 for observed reflections, residual electron density -0.23 to $0.17 \mathrm{e}^{-3}$. CCDC 1440643 contains the supplementary crystallographic data for this paper. These data can be obtained free of charge from The Cambridge Crystallographic Data Centre via www.ccdc.cam.ac.uk/data request/cif.

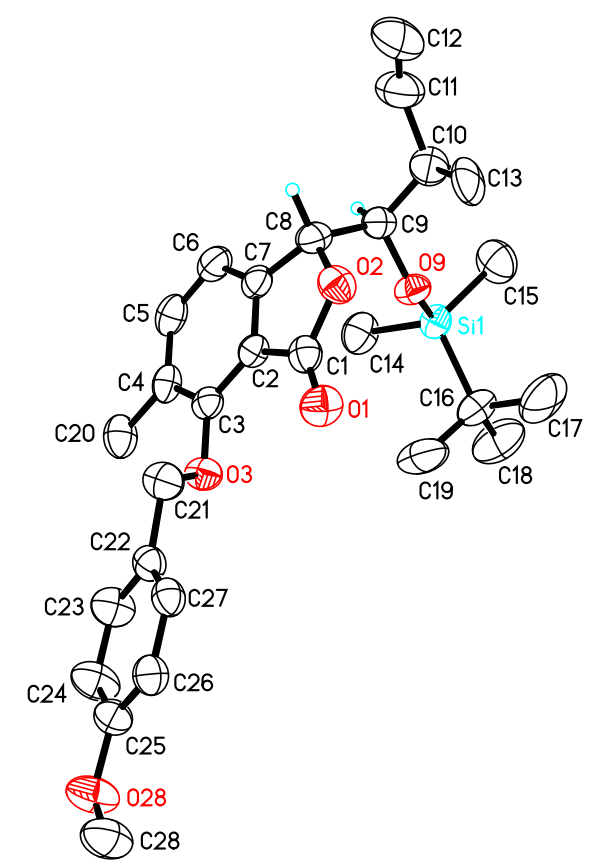

Thermal ellipsoid plots of compound $\mathbf{5 4}$ (50\% probability). 
Crystal data and structure refinement for $\mathbf{5 4}$.

\begin{tabular}{|c|c|c|}
\hline Identification code & \multicolumn{2}{|l|}{ CCDC 1440643} \\
\hline Empirical formula & \multicolumn{2}{|l|}{$\mathrm{C}_{28} \mathrm{H}_{38} \mathrm{O}_{5} \mathrm{Si}$} \\
\hline Formula weight & \multicolumn{2}{|l|}{482.67} \\
\hline Temperature & \multicolumn{2}{|l|}{$200(2) \mathrm{K}$} \\
\hline Wavelength & \multicolumn{2}{|l|}{$0.71073 \AA$} \\
\hline Crystal system & \multicolumn{2}{|l|}{ triclinic } \\
\hline Space group & \multicolumn{2}{|l|}{$\mathrm{P} \overline{1}$} \\
\hline $\mathrm{Z}$ & \multicolumn{2}{|c|}{2} \\
\hline Unit cell dimensions & $\begin{array}{l}\mathrm{a}=7.5558(10) \AA \\
\mathrm{b}=13.3993(17) \AA \\
\mathrm{c}=14.2195(18) \AA\end{array}$ & $\begin{array}{l}\alpha=83.276(3) \mathrm{deg} . \\
\beta=80.259(3) \mathrm{deg} . \\
\gamma=78.211(3) \mathrm{deg}\end{array}$ \\
\hline Volume & $1383.8(3) \AA^{3}$ & \\
\hline Density (calculated) & $1.16 \mathrm{~g} / \mathrm{cm}^{3}$ & \\
\hline Absorption coefficient & $0.12 \mathrm{~mm}^{-1}$ & \\
\hline Crystal shape & needle & \\
\hline Crystal size & $1.18 \times 0.15 \times 0.13$ & \\
\hline Crystal colour & colourless & \\
\hline Theta range for data collection & 1.6 to $24.8 \mathrm{deg}$. & \\
\hline Index ranges & $-8 \leq \mathrm{h} \leq 8,-15 \leq \mathrm{k} \leq 15$ & \\
\hline Reflections collected & 4614 & \\
\hline Independent reflections & $4614(\mathrm{R}(\mathrm{int})=0.00$ & \\
\hline Observed reflections & $3709(\mathrm{I}>2 \sigma(\mathrm{I}))$ & \\
\hline Absorption correction & Semi-empirical fro & ralents \\
\hline Max. and min. transmission & 0.98 and 0.87 & \\
\hline Refinement method & Full-matrix least-sc & $n \mathrm{~F}^{2}$ \\
\hline Data/restraints/parameters & 4614 / 5 / 346 & \\
\hline Goodness-of-fit on $\mathrm{F}^{2}$ & 1.14 & \\
\hline Final $R$ indices $(I>2 \sigma(I))$ & $\mathrm{R} 1=0.055, \mathrm{wR} 2=$ & \\
\hline Largest diff. peak and hole & 0.17 and $-0.23 \mathrm{e}^{-3}$ & \\
\hline
\end{tabular}

\section{X-ray crystal structure analysis of compound 57}

Description: colourless crystal (polyhedron), dimensions $0.48 \times 0.37 \times 0.11 \mathrm{~mm}^{3}$, crystal system orthorhombic, space group P2 $2_{1} 2_{1} 2_{1}, \mathrm{Z}=4, \mathrm{a}=11.406(3) \AA, \mathrm{b}=13.239(3) \AA, \mathrm{c}=23.993$ (7) $\AA$, alpha $=$ $90 \mathrm{deg}$, beta $=90 \mathrm{deg}$, gamma $=90 \mathrm{deg}, \mathrm{V}=3623.0(18) \AA^{3}$, rho $=1.060 \mathrm{~g} / \mathrm{cm}^{3}, \mathrm{~T}=200(2) \mathrm{K}, \mathrm{Theta}_{\max }$ $=28.28 \mathrm{deg}$, radiation Mo Kalpha, lambda $=0.71073 \AA$, 0.3 deg omega-scans with CCD area detector, 500 frames covering the asymmetric unit in reciprocal space with a mean redundancy of 2.1 and $92.4 \%$ completeness for a resolution of $0.75 \AA$, 10573 reflections measured, 7549 unique (R(int) = 0.0522), 5056 observed (I >2 $\sigma(\mathrm{I})$ ), intensities were corrected for Lorentz and polarization effects, an empirical absorption correction was applied using SADABS ${ }^{1}$ based on the Laue symmetry of the reciprocal space, $\mathrm{mu}=0.13 \mathrm{~mm}^{-1}, \mathrm{~T}_{\min }=0.94, \mathrm{~T}_{\max }=0.99$, structure solved by direct methods and refined against $\mathrm{F}^{2}$ with a Full-matrix least-squares algorithm using the SHELXTL (Version 2008/4) software package, ${ }^{1} 358$ parameters refined, hydrogen atoms were treated using appropriate riding models, except of $\mathrm{H} 8$ of the hydroxy-group, which was refined isotropically, Flack absolute structure parameter 0.16(12), goodness of fit 0.89 for observed reflections, final residual values $\mathrm{R} 1(\mathrm{~F})=0.053, \mathrm{wR}\left(\mathrm{F}^{2}\right)=0.110$ for observed reflections, residual electron density -0.23 to $0.24 \mathrm{e}^{-3}$. CCDC 1440644 contains the supplementary crystallographic data for this paper. These data can be obtained free of charge from The Cambridge Crystallographic Data Centre via www.ccdc.cam.ac.uk/data request/cif. 


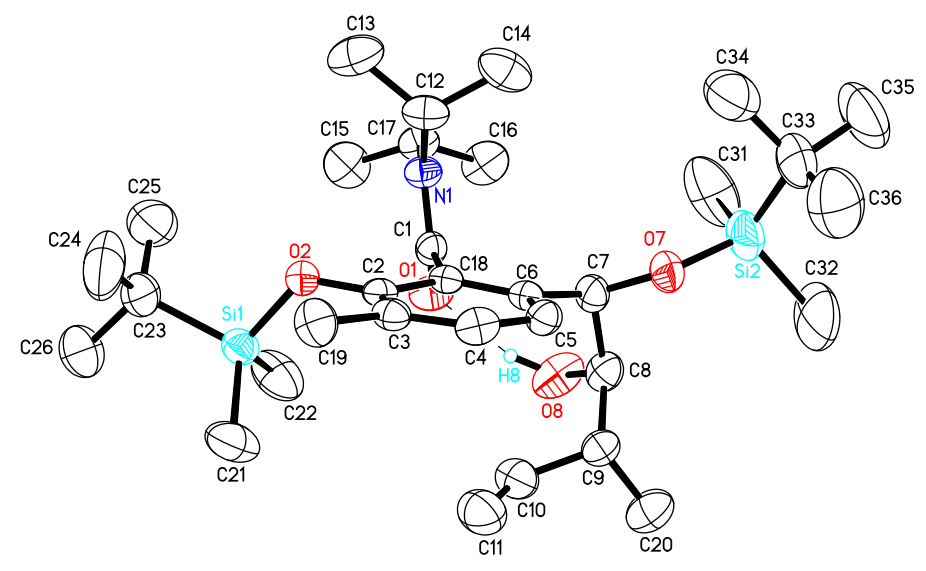

Thermal ellipsoid plots of compound $\mathbf{5 7}$ (50\% probability).

Table 10.12.1 Crystal data and structure refinement for 57 .

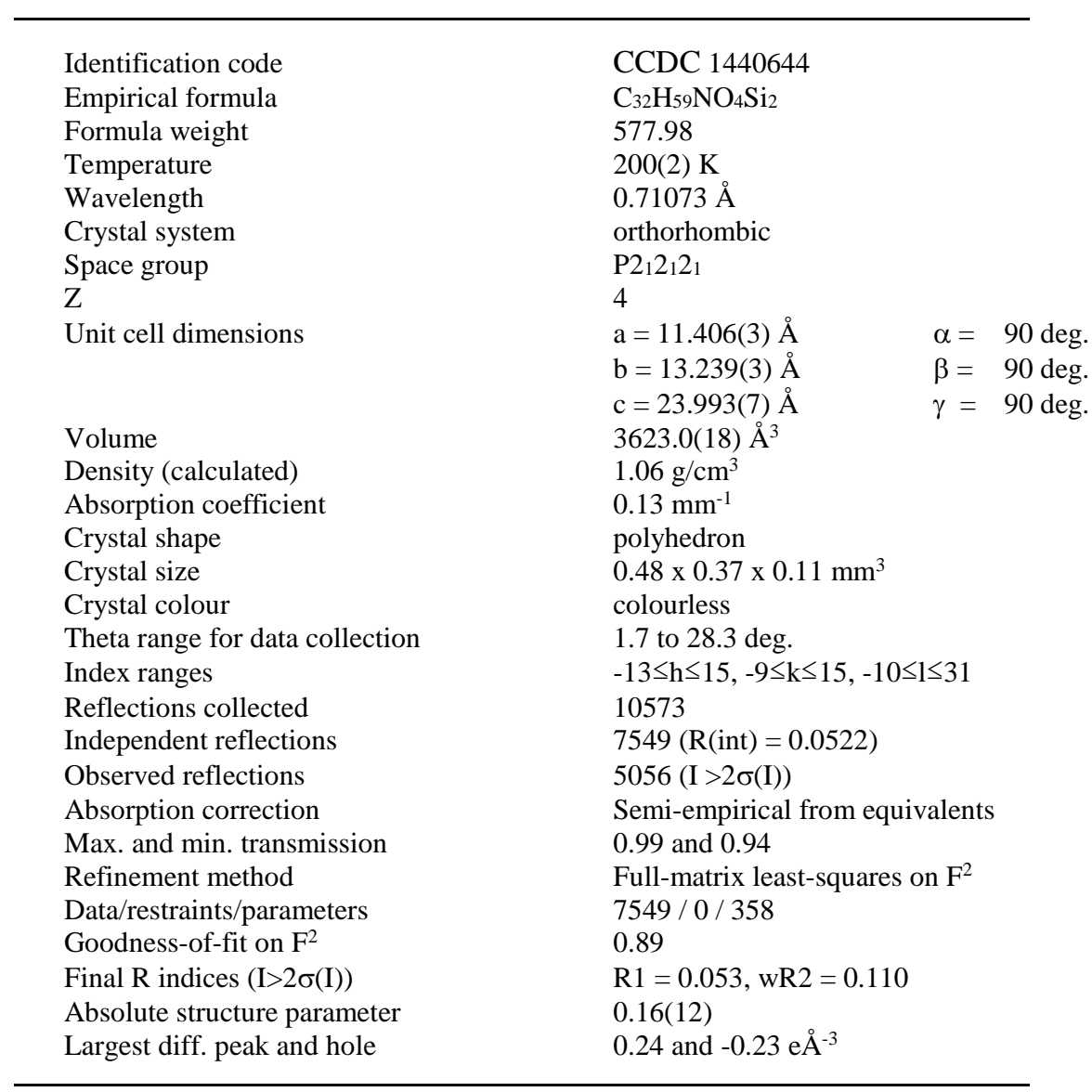

\section{X-ray crystal structure analysis of compound 67}

Description: colourless crystal (needle), dimensions $1.40 \times 0.11 \times 0.05 \mathrm{~mm}^{3}$, crystal system tetragonal, space group $\mathrm{P} 4{ }_{1} 2{ }_{1} 2, \mathrm{Z}=8, \mathrm{a}=15.600(4) \AA, \mathrm{b}=15.600(4) \AA, \mathrm{c}=22.648(7) \AA$, alpha $=90$ deg, beta $=$ $90 \mathrm{deg}$, gamma $=90 \mathrm{deg}, \mathrm{V}=5511(3) \AA^{3}$, rho $=1.043 \mathrm{~g} / \mathrm{cm}^{3}, \mathrm{~T}=200(2) \mathrm{K}$, Theta $\mathrm{T}_{\max }=23.00 \mathrm{deg}$, radiation Mo Kalpha, lambda $=0.71073 \AA, 0.3$ deg omega-scans with CCD area detector, covering a whole sphere in reciprocal space, 26690 reflections measured, 3839 unique ( $\mathrm{R}$ (int)=0.0615), 3086 
observed (I >2 $\sigma$ (I)), intensities were corrected for Lorentz and polarization effects, an empirical absorption correction was applied using SADABS based on the Laue symmetry of the reciprocal space, $\mathrm{mu}=0.07 \mathrm{~mm}^{-1}, \mathrm{~T}_{\min }=0.91, \mathrm{~T}_{\max }=1.00$, structure solved by direct methods and refined against $\mathrm{F}^{2}$ with a Full-matrix least-squares algorithm using the SHELXTL (Version 2008/4) software package, ${ }^{1} 283$ parameters refined, hydrogen atoms were treated using appropriate riding models, Flack absolute structure parameter 2(2), goodness of fit 1.05 for observed reflections, final residual values $R 1(F)=$ 0.058, wR $\left(\mathrm{F}^{2}\right)=0.150$ for observed reflections, residual electron density -0.15 to $0.32 \mathrm{e}^{-3}$. CCDC 907660 contains the supplementary crystallographic data for this paper. These data can be obtained free of charge from The Cambridge Crystallographic Data Centre via www.ccdc.cam.ac.uk/data_request/cif.

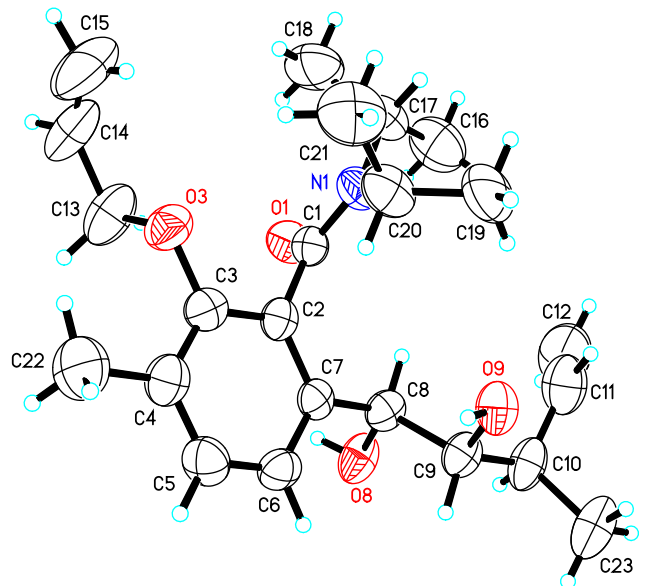

Thermal ellipsoid plots of compound 67 (50\% probability).

Crystal data and structure refinement for 67.

Identification code

Empirical formula

Formula weight

Temperature

Wavelength

Crystal system

Space group

Z

Unit cell dimensions

Volume

Density (calculated)

Absorption coefficient

Crystal shape

Crystal size

Crystal colour

Theta range for data collection

Index ranges

Reflections collected

Independent reflections

Observed reflections

Absorption correction

Max. and min. transmission

Refinement method

Data/restraints/parameters

Goodness-of-fit on $\mathrm{F}^{2}$

Final R indices $(\mathrm{I}>2 \sigma(\mathrm{I}))$

Absolute structure parameter

Largest diff. peak and hole

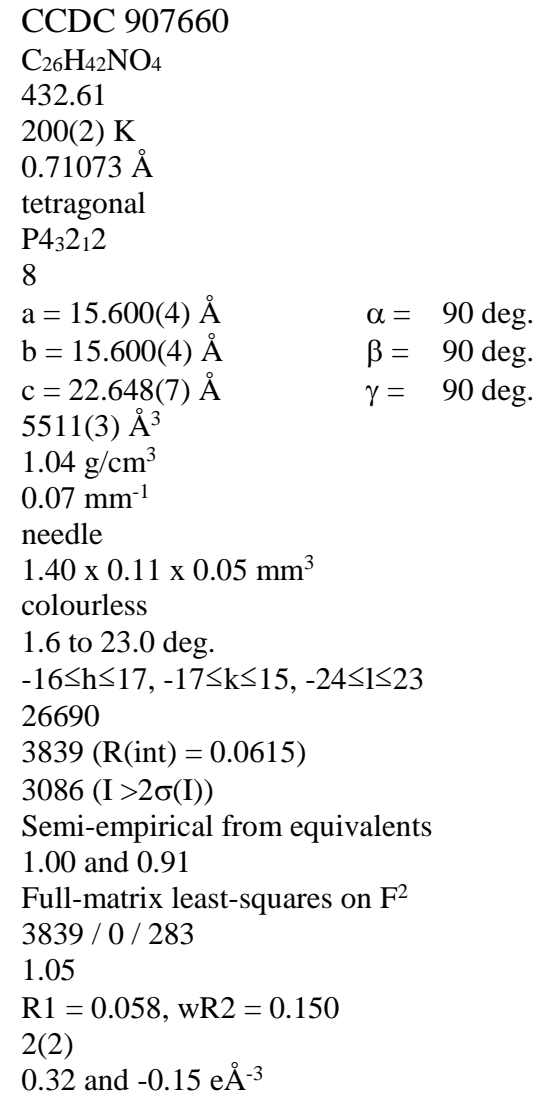




\section{X-ray crystal structure analysis of compound 41}

Description: colourless crystal (plate), dimensions $0.27 \times 0.18 \times 0.05 \mathrm{~mm}^{3}$, crystal system orthorhombic, space group $\mathrm{P} 2{ }_{2} 2{ }_{1}{ }_{1}, \mathrm{Z}=8, \mathrm{a}=11.4204(8) \AA, \mathrm{b}=17.2635(13) \AA, \mathrm{c}=33.066(3) \AA$, alpha $=90$ deg, beta $=90 \mathrm{deg}$, gamma=90 deg, $\mathrm{V}=6519.2(9) \AA^{3}, \mathrm{rho}=1.033 \mathrm{~g} / \mathrm{cm}^{3}, \mathrm{~T}=200(2) \mathrm{K}$, Theta $\mathrm{max}=20.83 \mathrm{deg}$, radiation Mo Kalpha, lambda $=0.71073 \AA$, 0.3 deg omega-scans with CCD area detector, covering the asymmetric unit in reciprocal space with a mean redundancy of 7.31and a completeness of $99.6 \%$ to a resolution of $1.00 \AA$, 28366 reflections measured, 6816 unique (R(int) $=0.0438), 5732$ observed (I > $2 \sigma$ (I)), intensities were corrected for Lorentz and polarization effects, an empirical absorption correction was applied using SADABS based on the Laue symmetry of the reciprocal space, $\mathrm{mu}=0.10 \mathrm{~mm}^{-1}, \mathrm{~T}_{\min }=$ $0.97, \mathrm{~T}_{\max }=1.00$, structure solved by direct methods and refined against $\mathrm{F}^{2}$ with a Full-matrix leastsquares algorithm using the SHELXTL (Version 2008/4) software package, ${ }^{1} 649$ parameters refined, hydrogen atoms were treated using appropriate riding models, except of H3 and H9 of the hydroxy groups, which were refined isotropically, Flack absolute structure parameter 0.07(17), goodness of fit 1.05 for observed reflections, final residual values $\mathrm{R} 1(\mathrm{~F})=0.044, \mathrm{wR}\left(\mathrm{F}^{2}\right)=0.100$ for observed reflections, residual electron density -0.18 to $0.24 \mathrm{e}^{-3}$. CCDC 907658 contains the supplementary crystallographic data for this paper. These data can be obtained free of charge from The Cambridge Crystallographic Data Centre via www.ccdc.cam.ac.uk/data_request/cif.

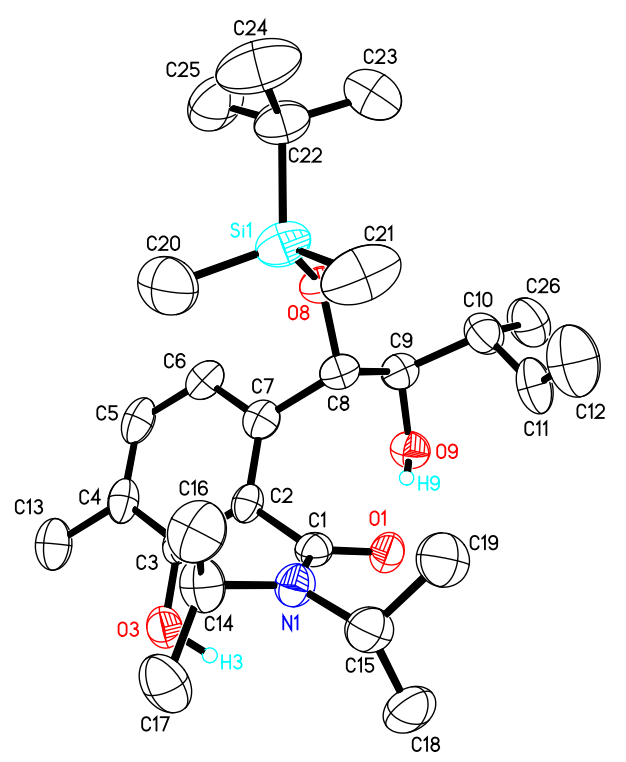

Thermal ellipsoid plots of compound $\mathbf{4 1}$ (50\% probability). 
Crystal data and structure refinement for $\mathbf{4 1 .}$

\begin{tabular}{|c|c|c|c|}
\hline Identification code & \multicolumn{3}{|l|}{ CCDC 907658} \\
\hline Empirical formula & \multicolumn{3}{|l|}{$\mathrm{C}_{29} \mathrm{H}_{52} \mathrm{NO}_{4} \mathrm{Si}$} \\
\hline Formula weight & \multicolumn{3}{|l|}{506.81} \\
\hline Temperature & \multicolumn{3}{|l|}{$200(2) \mathrm{K}$} \\
\hline Wavelength & \multicolumn{3}{|l|}{$0.71073 \AA$} \\
\hline Crystal system & \multicolumn{3}{|l|}{ orthorhombic } \\
\hline Space group & \multicolumn{3}{|l|}{$\mathrm{P} 2{ }_{1} 2{ }_{2}{ }_{1}$} \\
\hline $\mathrm{Z}$ & \multicolumn{3}{|l|}{8} \\
\hline \multirow[t]{3}{*}{ Unit cell dimensions } & $\mathrm{a}=11.4204(8) \AA$ & $\alpha=$ & $90 \mathrm{deg}$. \\
\hline & $\mathrm{b}=17.2635(13) \AA$ & $\beta=$ & $90 \mathrm{deg}$. \\
\hline & $c=33.066(3) \AA$ & $\gamma=$ & \\
\hline Volume & $6519.2(9) \AA^{3}$ & & \\
\hline Density (calculated) & $1.03 \mathrm{~g} / \mathrm{cm}^{3}$ & & \\
\hline Absorption coefficient & $0.10 \mathrm{~mm}^{-1}$ & & \\
\hline Crystal shape & plate & & \\
\hline Crystal size & $0.27 \times 0.18 \times 0.05$ & & \\
\hline Crystal colour & colourless & & \\
\hline Theta range for data collection & 1.3 to $20.8 \mathrm{deg}$. & & \\
\hline Index ranges & $-11 \leq \mathrm{h} \leq 11,-17 \leq \mathrm{k} \leq 1$ & $\leq 1 \leq 33$ & \\
\hline Reflections collected & 28366 & & \\
\hline Independent reflections & $6816(\mathrm{R}(\mathrm{int})=0.04$ & & \\
\hline Observed reflections & $5732(\mathrm{I}>2 \sigma(\mathrm{I}))$ & & \\
\hline Absorption correction & Semi-empirical fro & & \\
\hline Max. and min. transmission & 1.00 and 0.97 & & \\
\hline Refinement method & Full-matrix least-sc & on $\mathrm{F}^{2}$ & \\
\hline Data/restraints/parameters & $6816 / 0 / 649$ & & \\
\hline Goodness-of-fit on $\mathrm{F}^{2}$ & 1.05 & & \\
\hline Final $R$ indices $(I>2 \sigma(I))$ & $\mathrm{R} 1=0.044, \mathrm{wR} 2=$ & & \\
\hline Absolute structure parameter & $0.07(17)$ & & \\
\hline Largest diff. peak and hole & 0.24 and $-0.18 \mathrm{e}^{-3}$ & & \\
\hline
\end{tabular}

\section{X-ray crystal structure analysis of compound 72}

Description: colourless crystal (polyhedron), dimensions $0.27 \times 0.15 \times 0.12 \mathrm{~mm}^{3}$, crystal system monoclinic, space group $\mathrm{P} 2{ }_{1}, \mathrm{Z}=8, \mathrm{a}=14.6819(6) \AA \mathrm{,}, \mathrm{b}=27.0915(11) \AA, \mathrm{c}=15.1614(7) \AA$, alpha $=90$ deg, beta $=91.392(1) \mathrm{deg}$, gamma $=90 \mathrm{deg}, \mathrm{V}=6028.7(4) \AA^{3}$, rho $=1.051 \mathrm{~g} / \mathrm{cm}^{3}, \mathrm{~T}=200(2) \mathrm{K}$, Theta $_{\max }=23.26 \mathrm{deg}$, radiation Mo Kalpha, lambda $=0.71073 \AA$, 0.5 deg omega-scans with CCD area detector, covering the asymmetric unit in reciprocal space with a mean redundancy of 6.05 and a completeness of $99.8 \%$ to a resolution of $0.90 \AA, 53802$ reflections measured, 17224 unique (R(int) = 0.0293), 12618 observed ( $>2 \sigma(\mathrm{I})$ ), intensities were corrected for Lorentz and polarization effects, an empirical absorption correction was applied using SADABS, based on the Laue symmetry of the reciprocal space, $\mathrm{mu}=0.14 \mathrm{~mm}^{-1} \mathrm{~T}_{\min }=0.96, \mathrm{~T}_{\max }=0.98$, structure solved by direct methods and refined against $\mathrm{F}^{2}$ with a Full-matrix least-squares algorithm using the SHELXTL (Version 2008/4) software package, ${ }^{1} 1387$ parameters refined, hydrogen atoms were treated using appropriate riding models, Flack absolute structure parameter $-0.03(11)$, goodness of fit 1.01 for observed reflections, final residual values $\mathrm{R} 1(\mathrm{~F})=0.058, \mathrm{wR}\left(\mathrm{F}^{2}\right)=0.143$ for observed reflections, residual electron density -0.33 to $0.48 \mathrm{e} \AA^{-}$ 3. CCDC 907659 contains the supplementary crystallographic data for this paper. These data can be obtained free of charge from The Cambridge Crystallographic Data Centre via www.ccdc.cam.ac.uk/data_request/cif. 


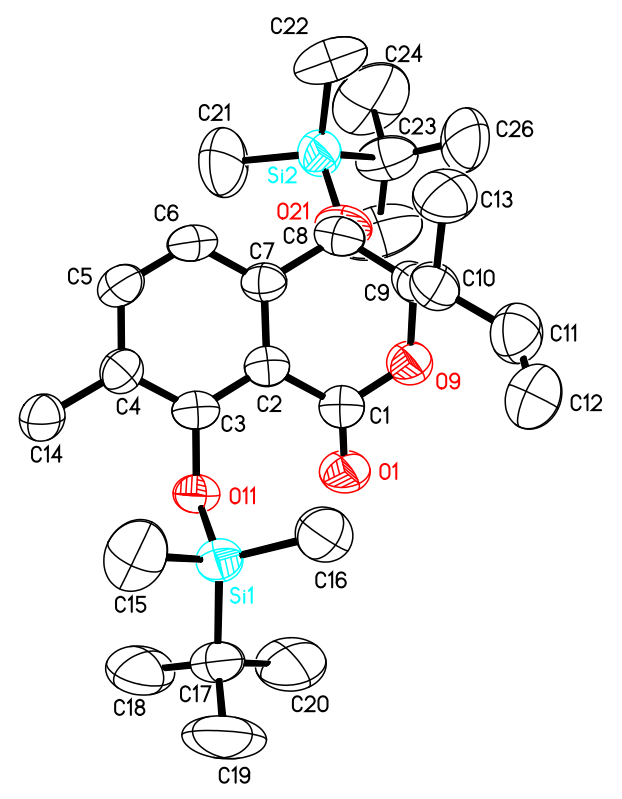

Thermal ellipsoid plots of compound $\mathbf{7 2}$ (50\% probability).

Crystal data and structure refinement for $\mathbf{7 2}$.

Identification code
Empirical formula
Formula weight
Temperature
Wavelength
Crystal system
Space group
Z
Unit cell dimensions

\section{Volume}

Density (calculated)

Absorption coefficient

Crystal shape

Crystal size

Crystal colour

Theta range for data collection

Index ranges

Reflections collected

Independent reflections

Observed reflections

Absorption correction

Max. and min. transmission

Refinement method

Data/restraints/parameters

Goodness-of-fit on $\mathrm{F}^{2}$

Final R indices $(\mathrm{I}>2 \sigma(\mathrm{I}))$

Absolute structure parameter

Largest diff. peak and hole

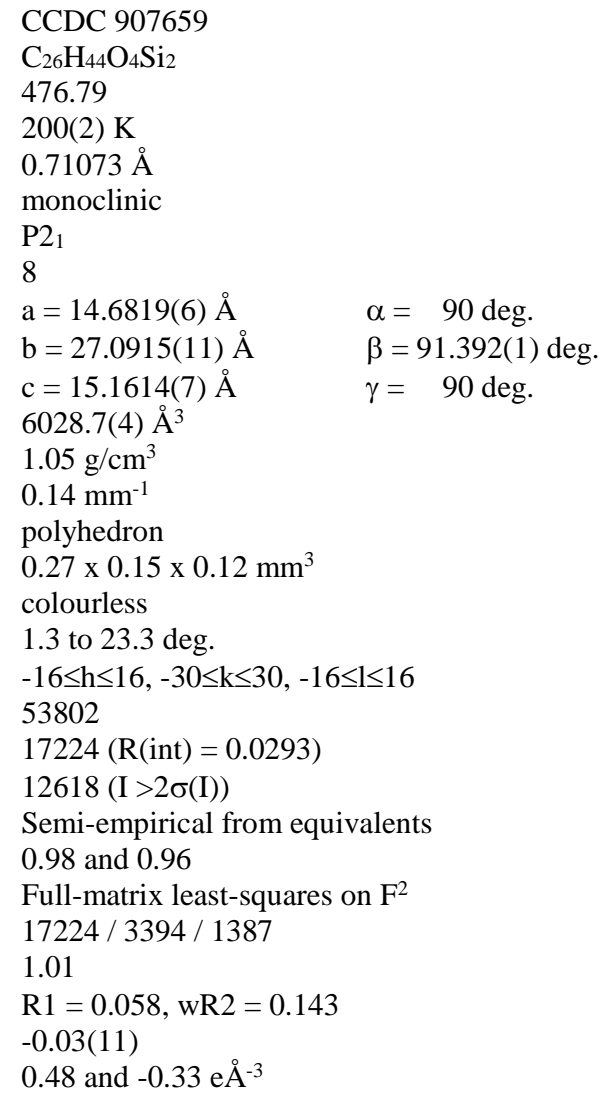




\section{References}

(1) Sheldrick, G.M. Acta Cryst. 2008, A64, 112-122. 
III Copies of ${ }^{1} \mathrm{H}$ - and ${ }^{13} \mathrm{C}-\mathrm{NMR}$ spectra

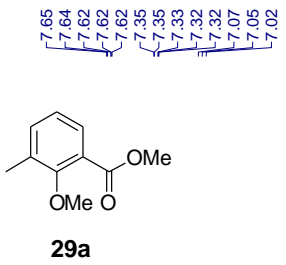

( $\left.{ }^{\mathrm{H}} \mathrm{NMR}, 300 \mathrm{MHz}\right)$
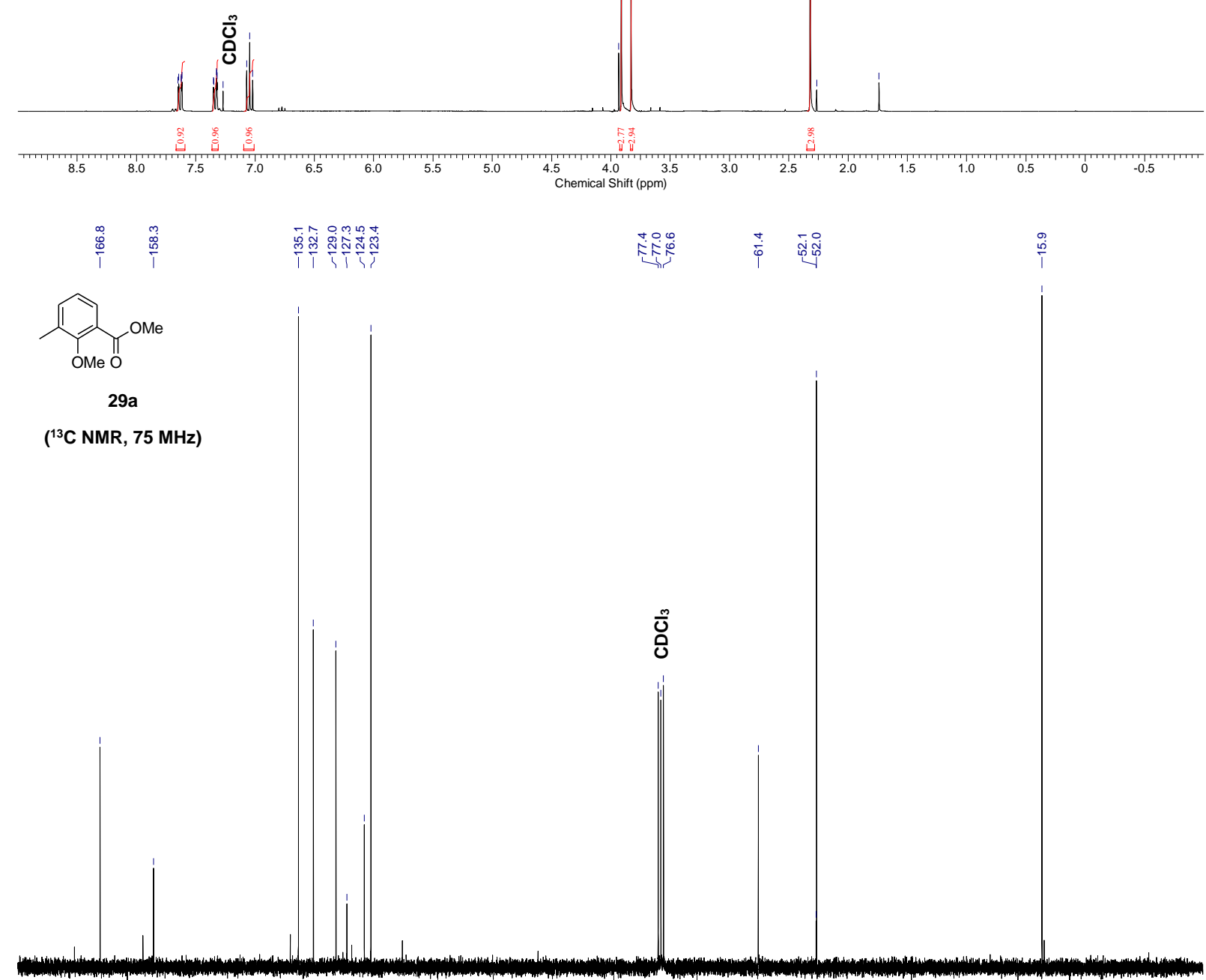

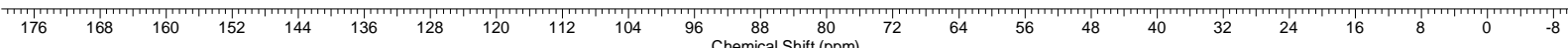


Supporting Information

11

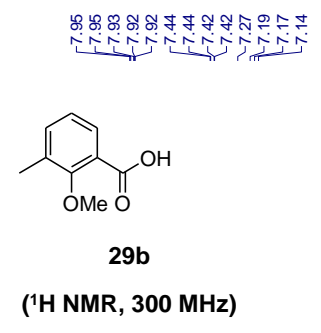

พิบับิ
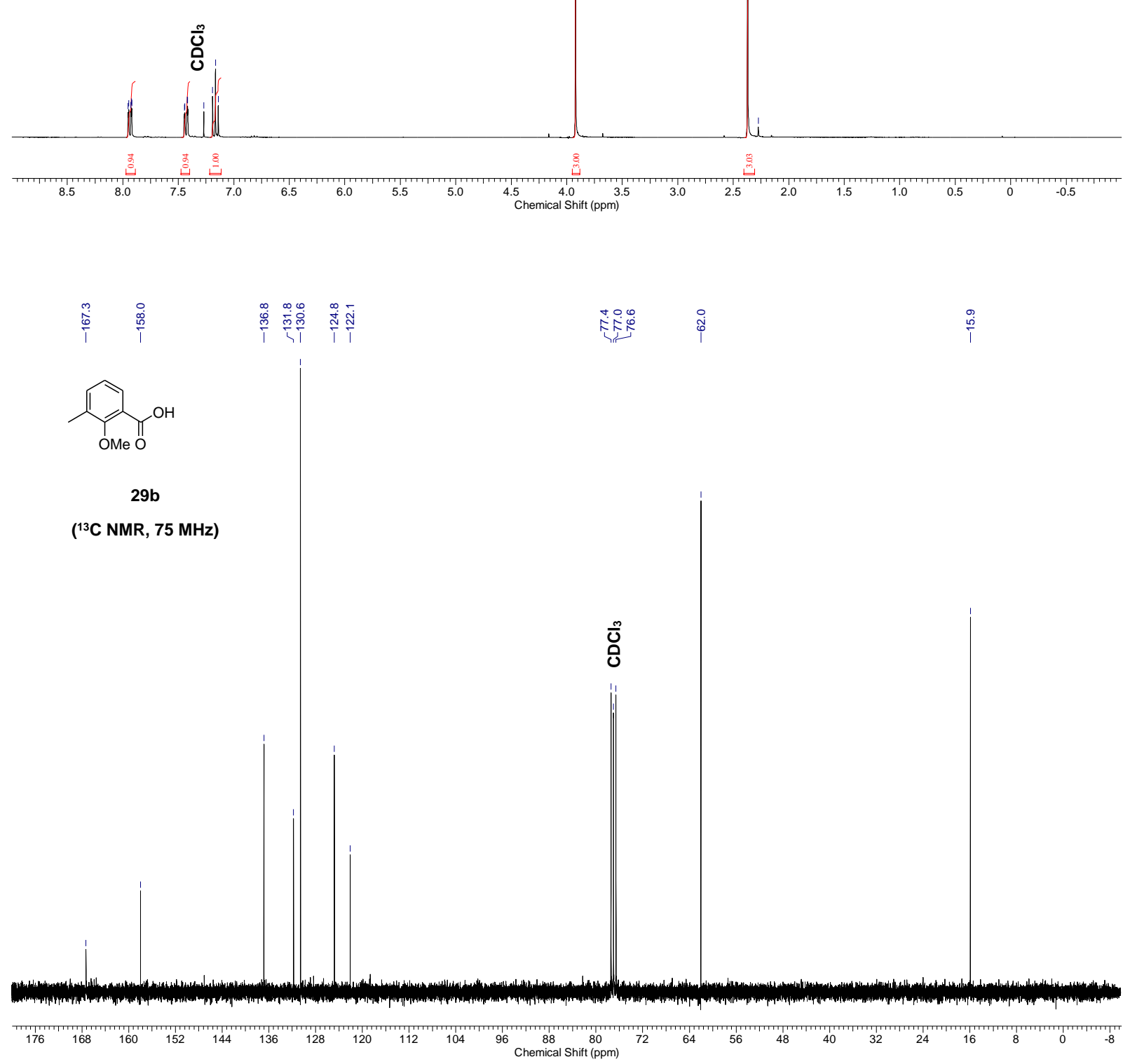


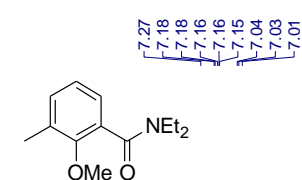

19

('H NMR, 300 MHz)

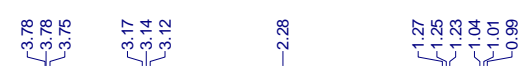
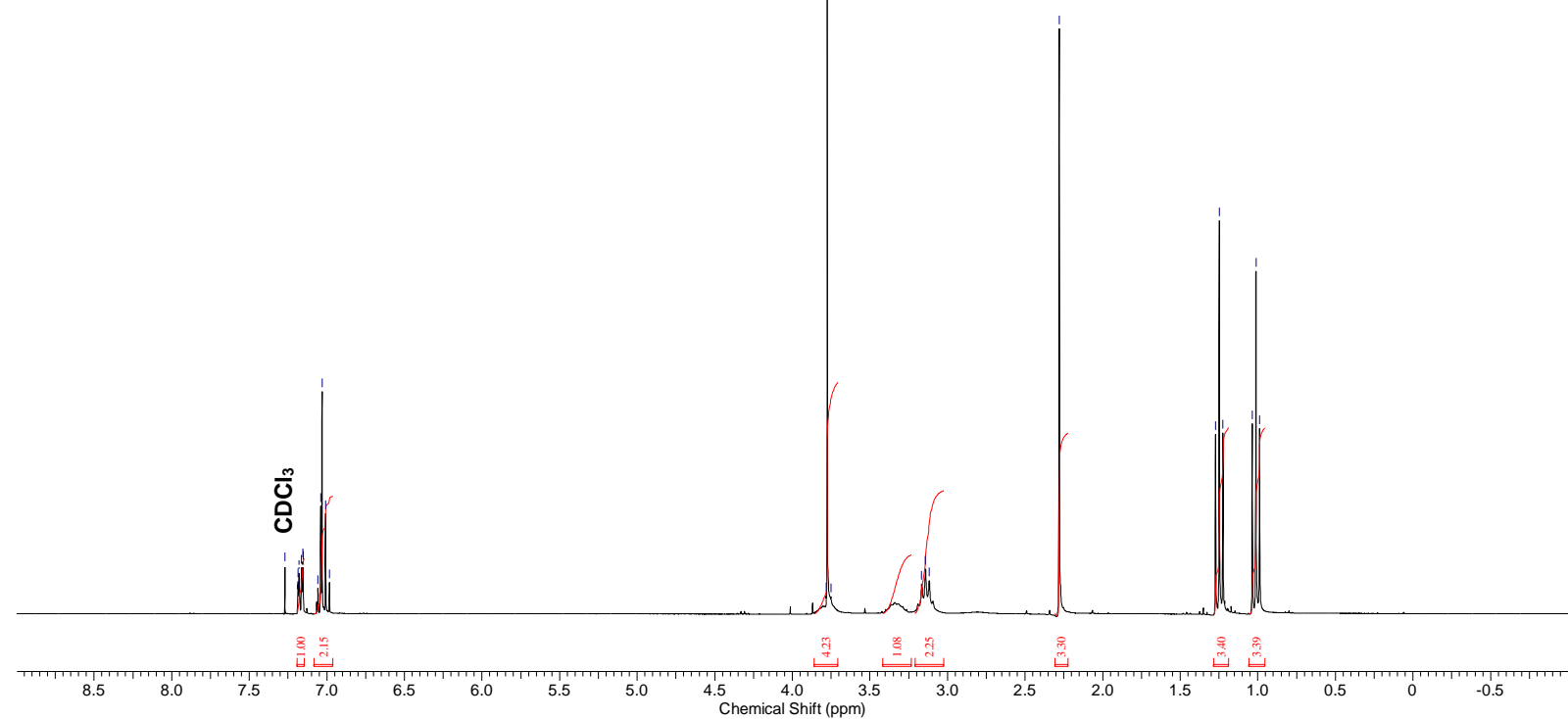

$\begin{array}{lll}1 & 1 \\ 0 & 0\end{array}$

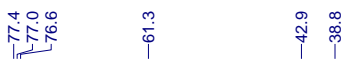

$\stackrel{i}{i} \dot{i} \hat{i}$<smiles>CCNC(=O)c1cccc(C)c1OC</smiles>

19

( ${ }^{13} \mathrm{C}$ NMR, $75 \mathrm{MHz}$ )

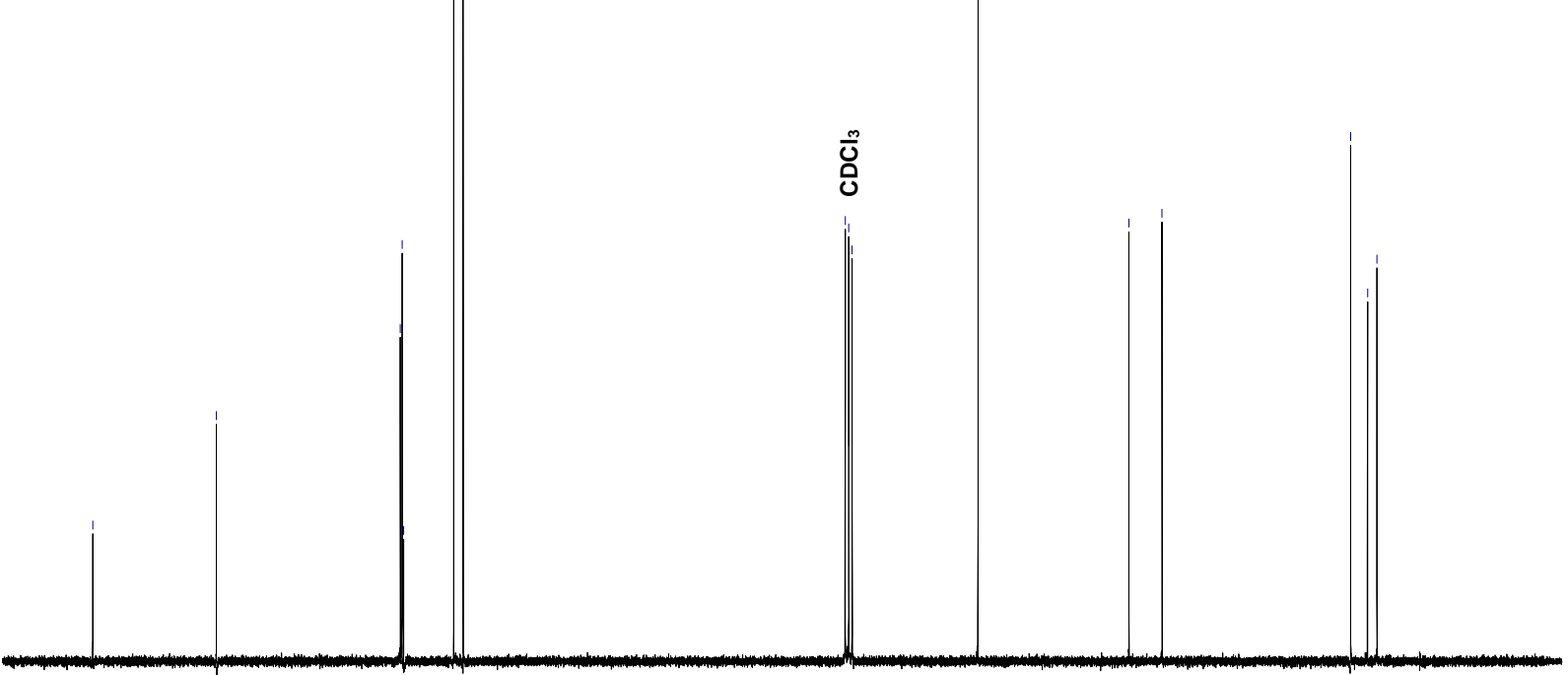

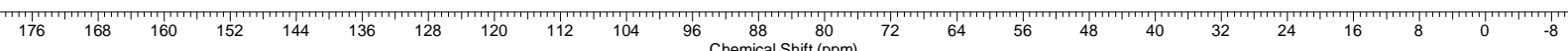




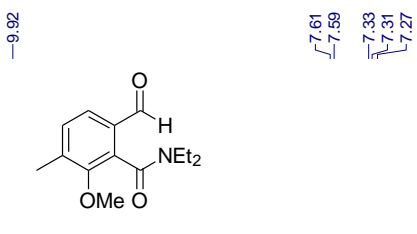

20

('H NMR, 300 MHz)
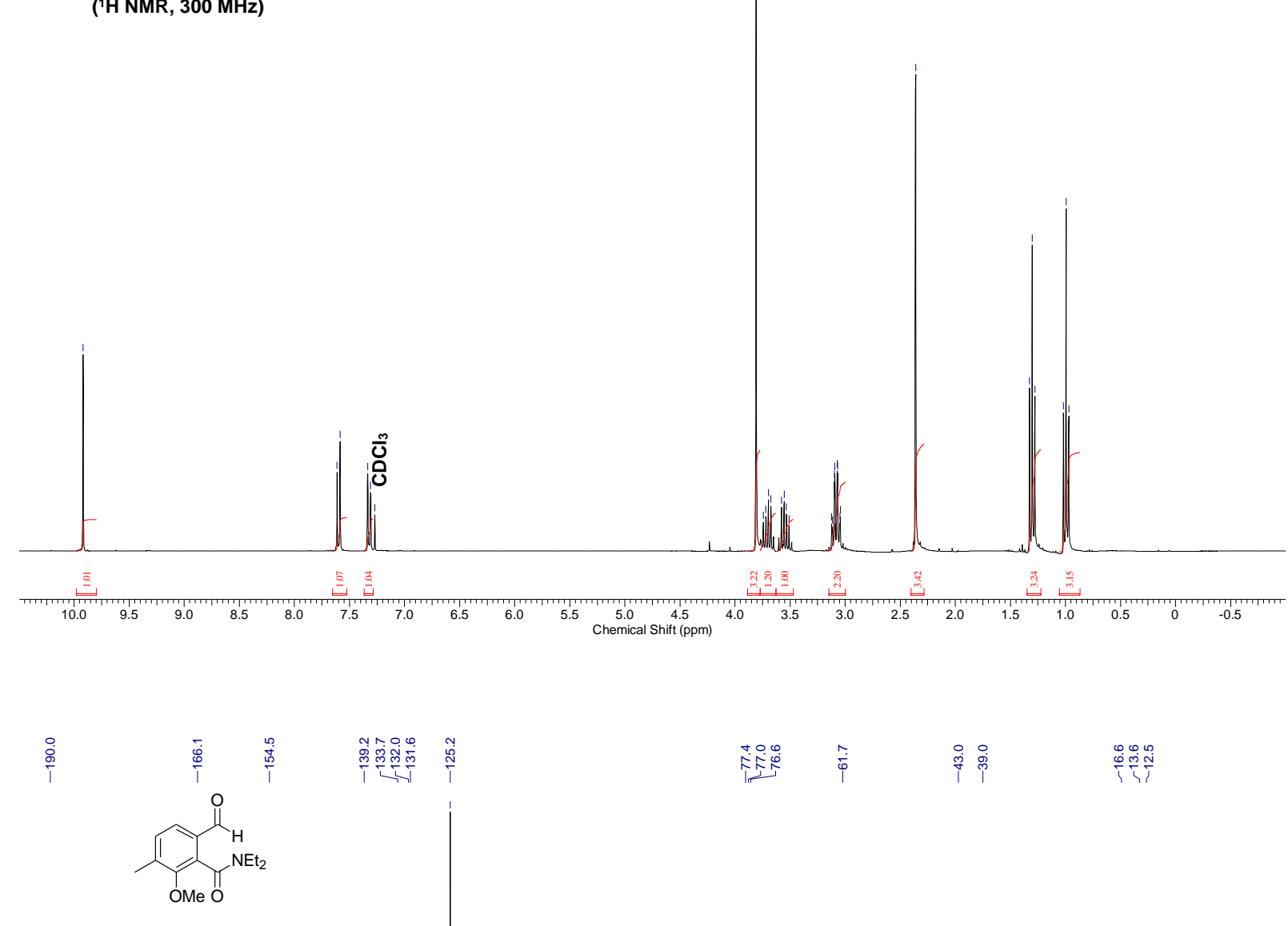

( ${ }^{13} \mathrm{C}$ NMR, $\left.75 \mathrm{MHz}\right)$

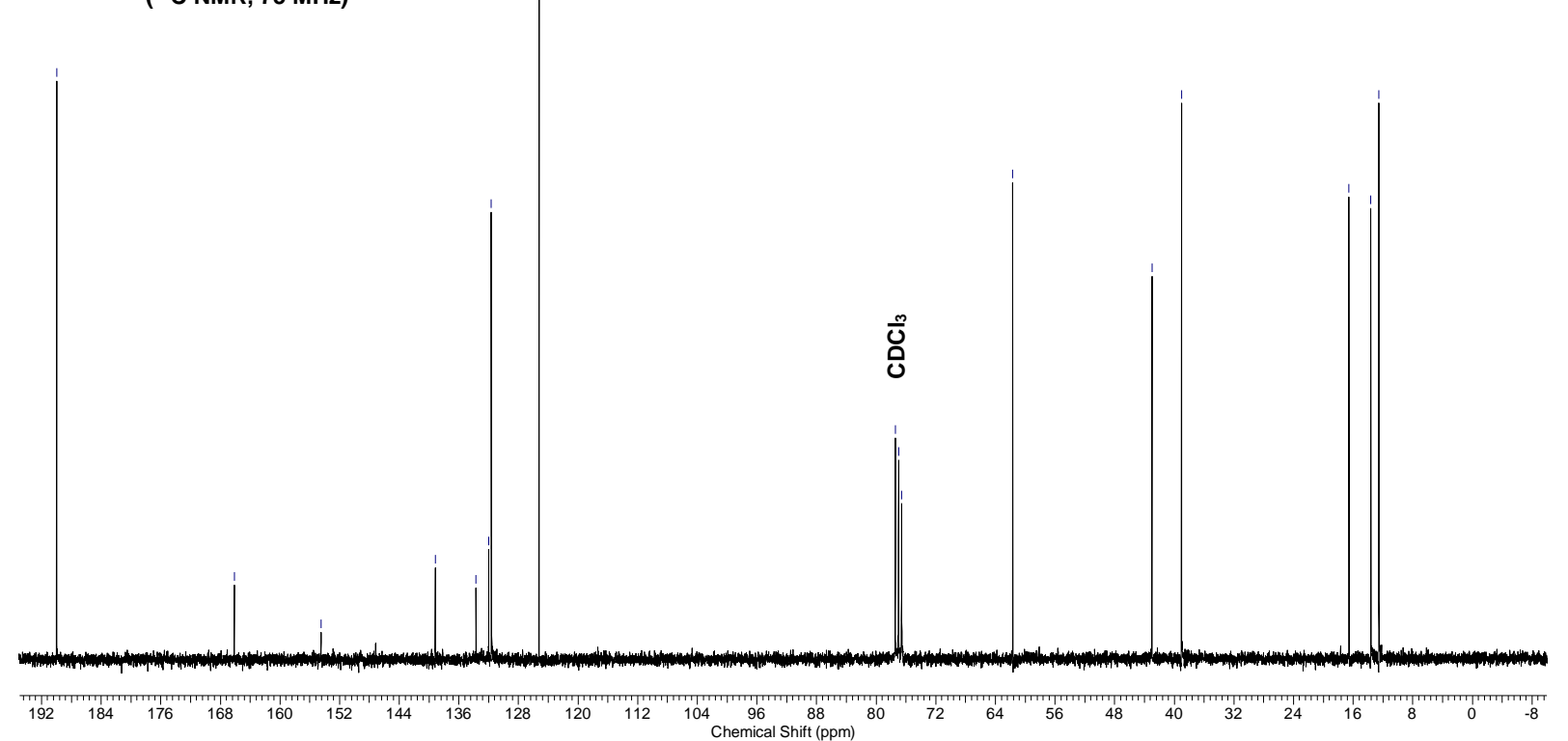




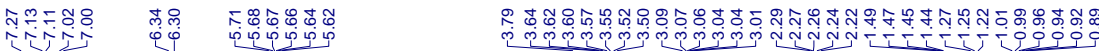<smiles>CCC/C=C\c1ccc(C)c(OC)c1C(=O)N(CC)CC</smiles>

21

('H NMR, 300 MHz)

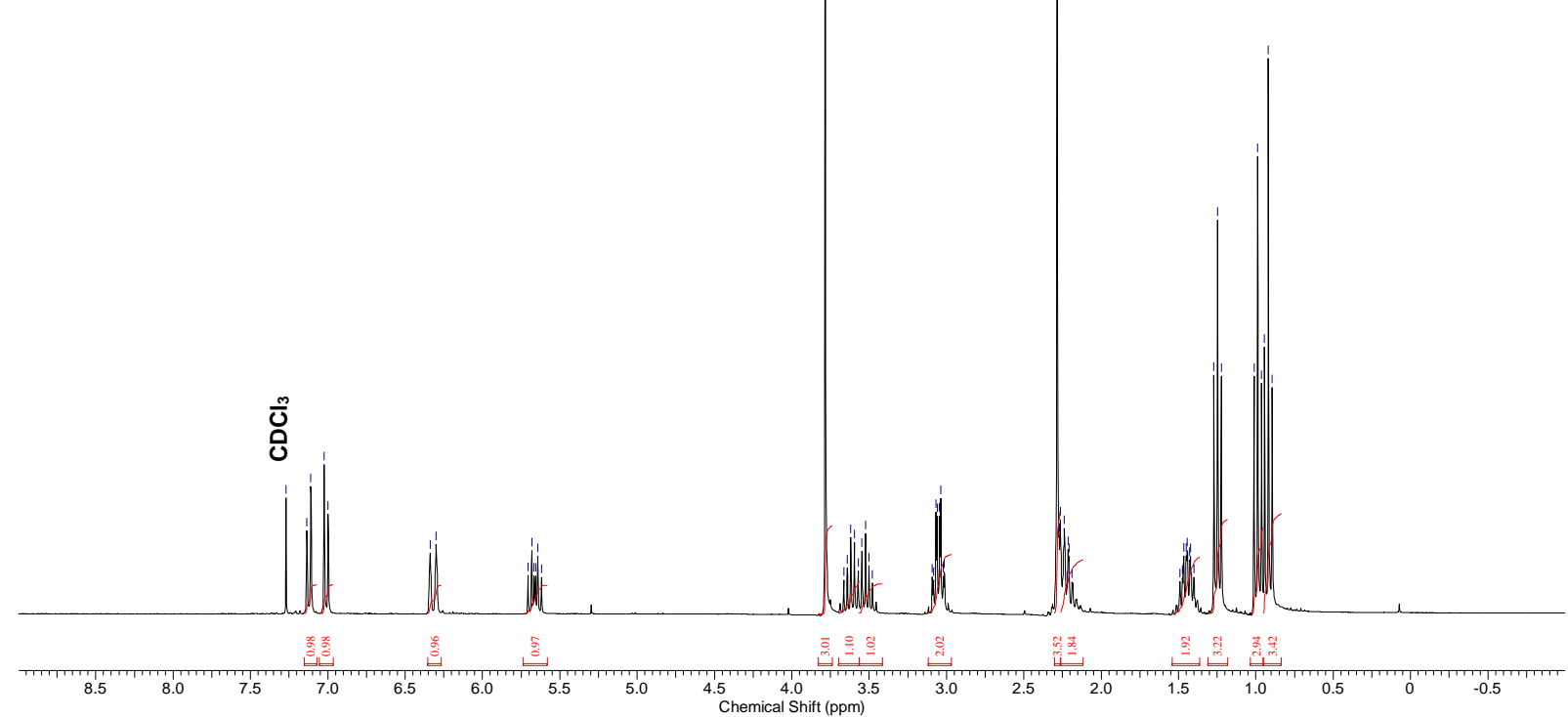
$\stackrel{\infty}{i}$

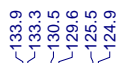

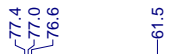

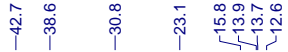

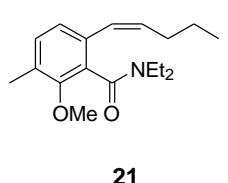

$\left({ }^{13} \mathrm{C}\right.$ NMR, $\left.75 \mathrm{MHz}\right)$
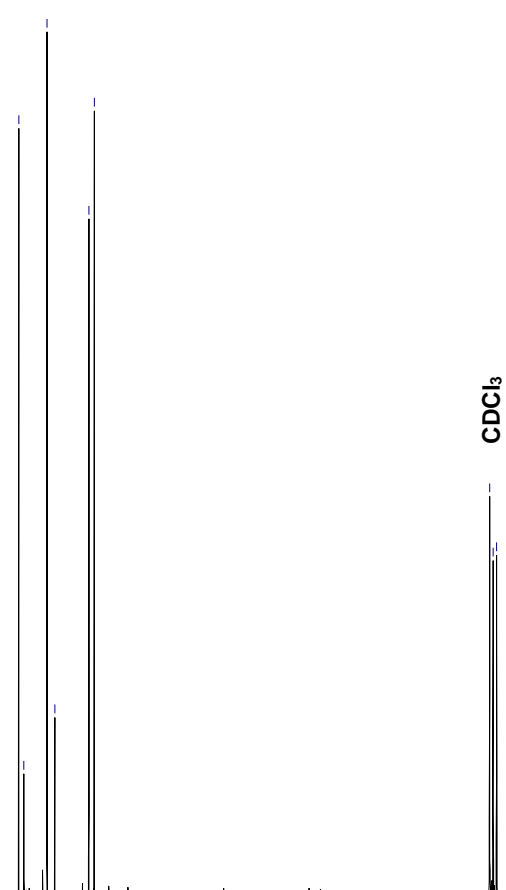

రั๊ 


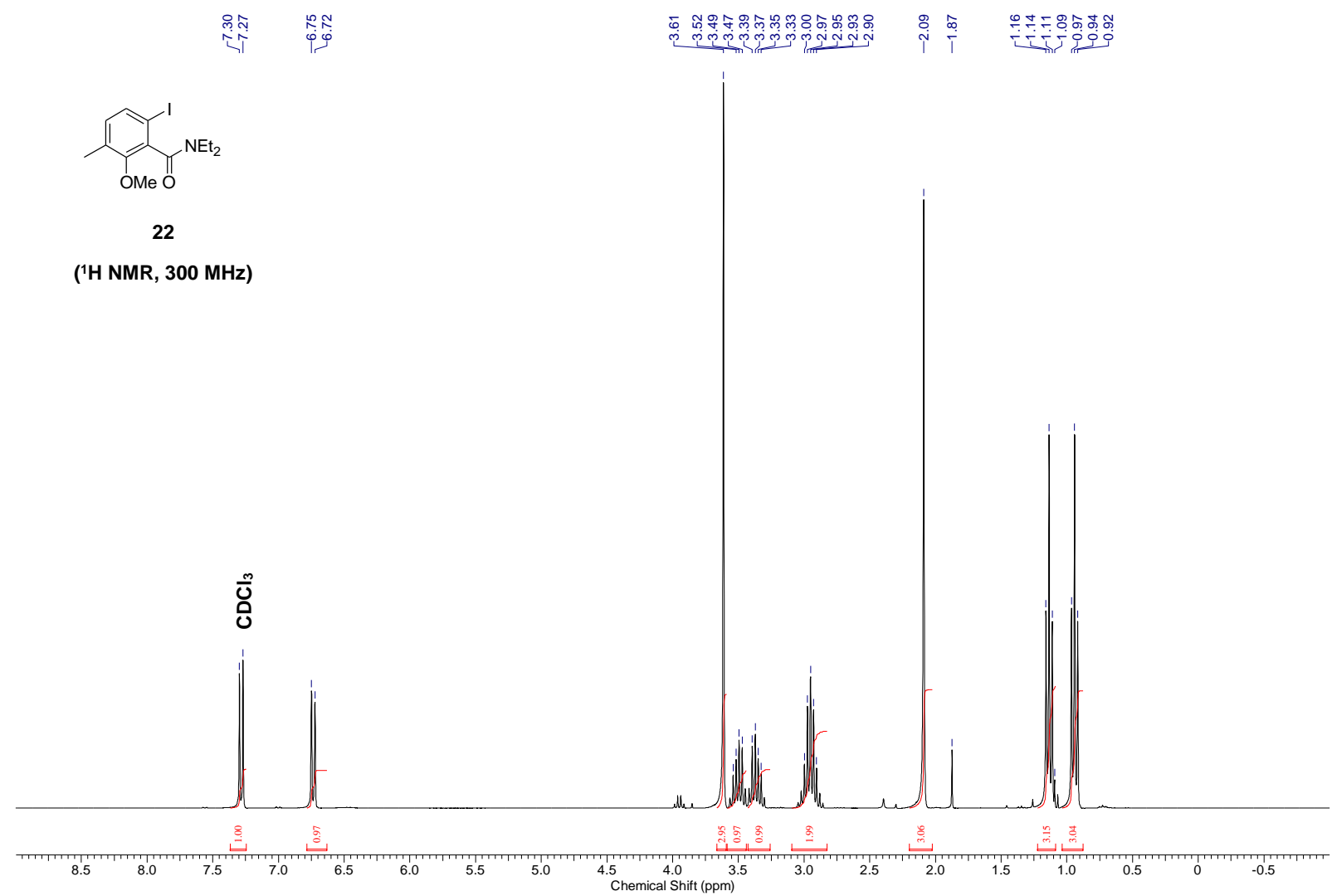

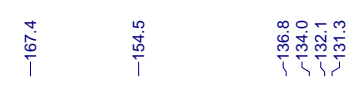

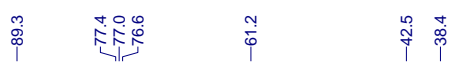

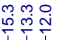

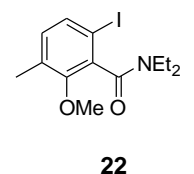

( ${ }^{13} \mathrm{C}$ NMR, $75 \mathrm{MHz}$ )

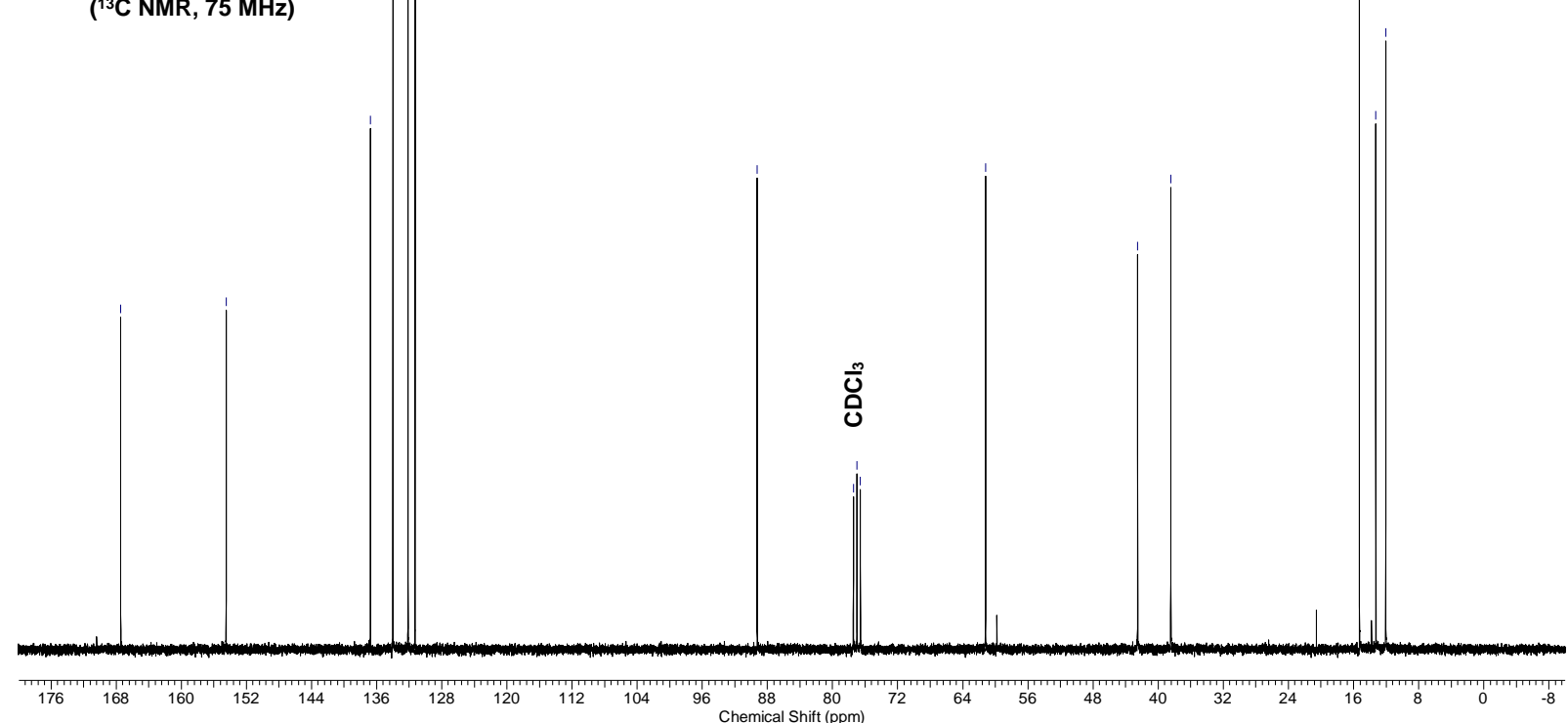




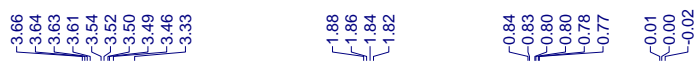
HO

23a

('H NMR, 300 MHz)

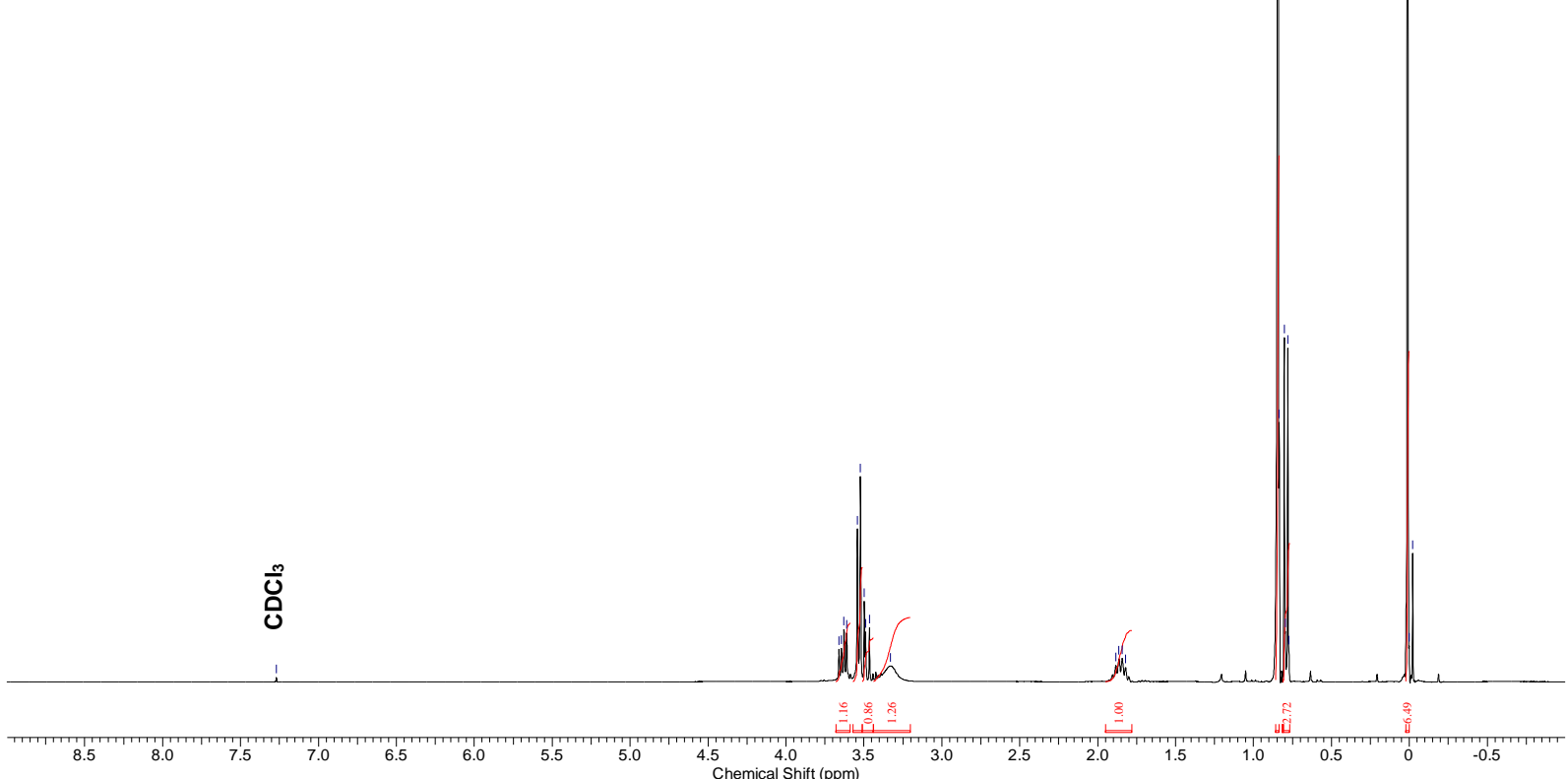

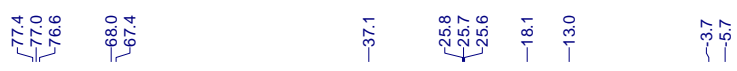

HO NotBs

23a

( ${ }^{13} \mathrm{C}$ NMR, $75 \mathrm{MHz}$ )

융

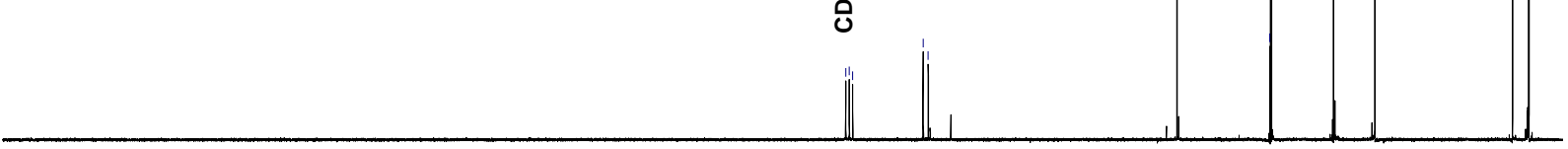

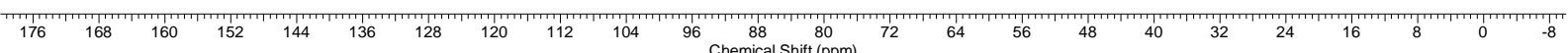




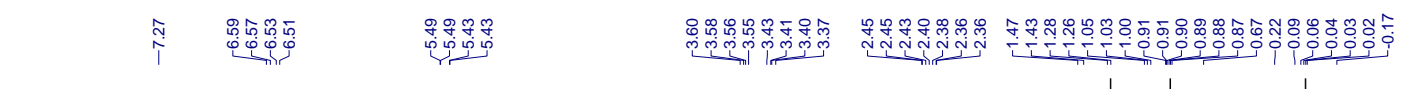

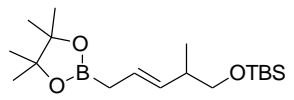

23

('H NMR, $300 \mathrm{MHz})$

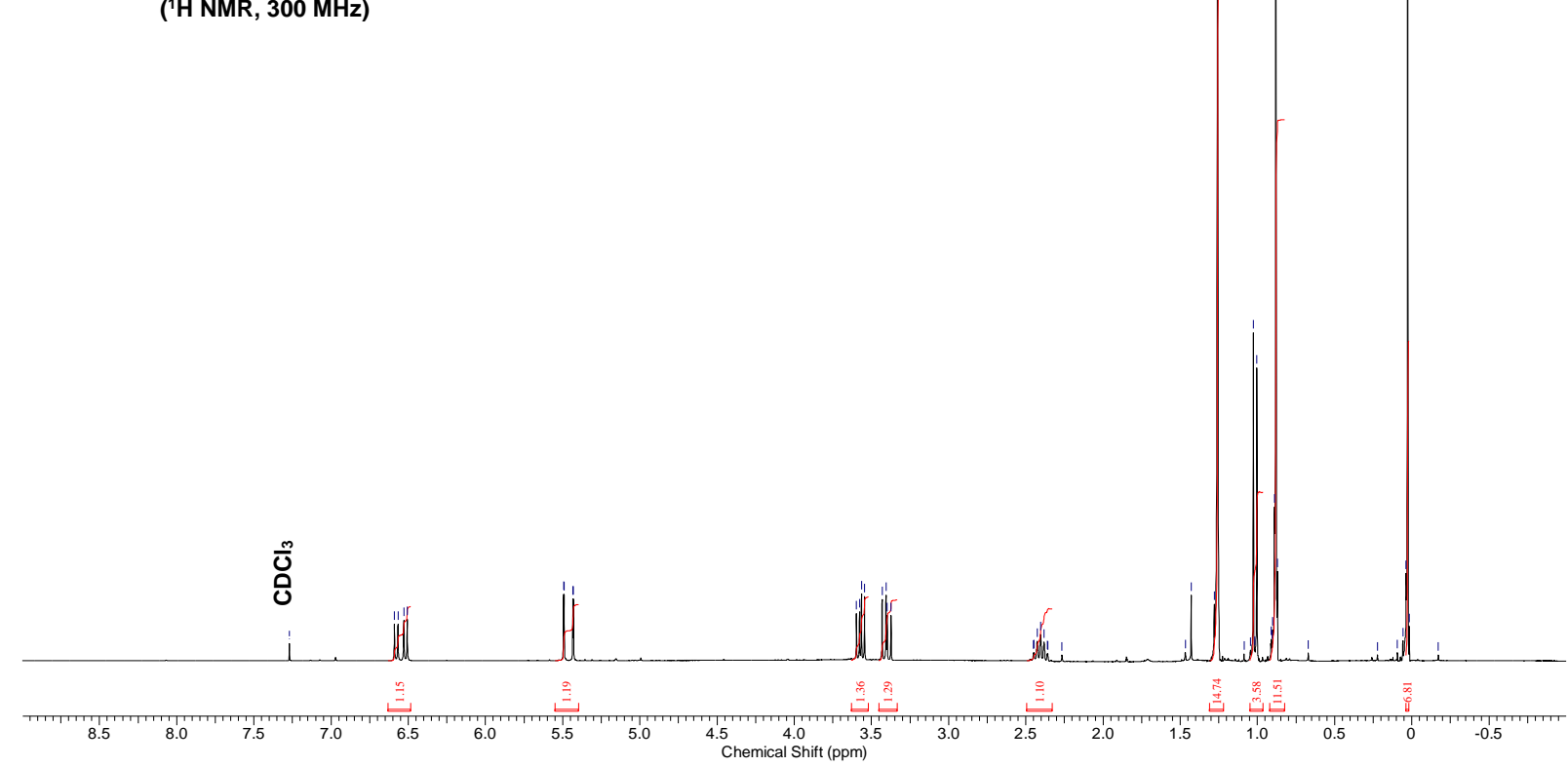

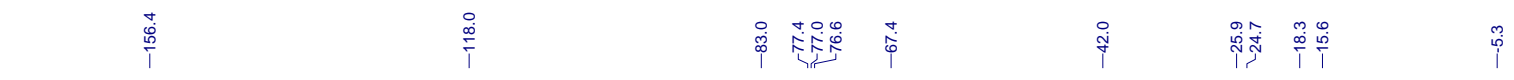

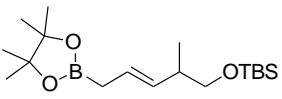

23

( ${ }^{13} \mathrm{C}$ NMR, $75 \mathrm{MHz}$ )

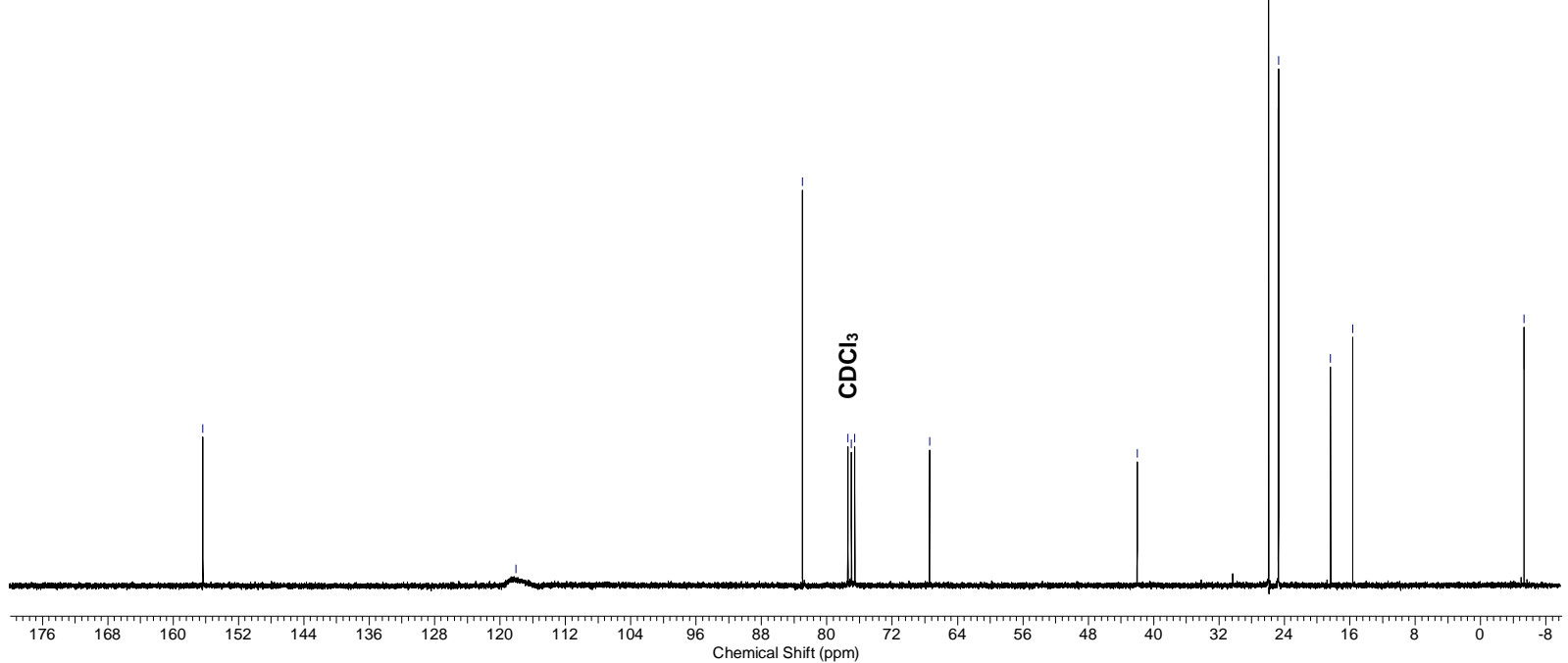




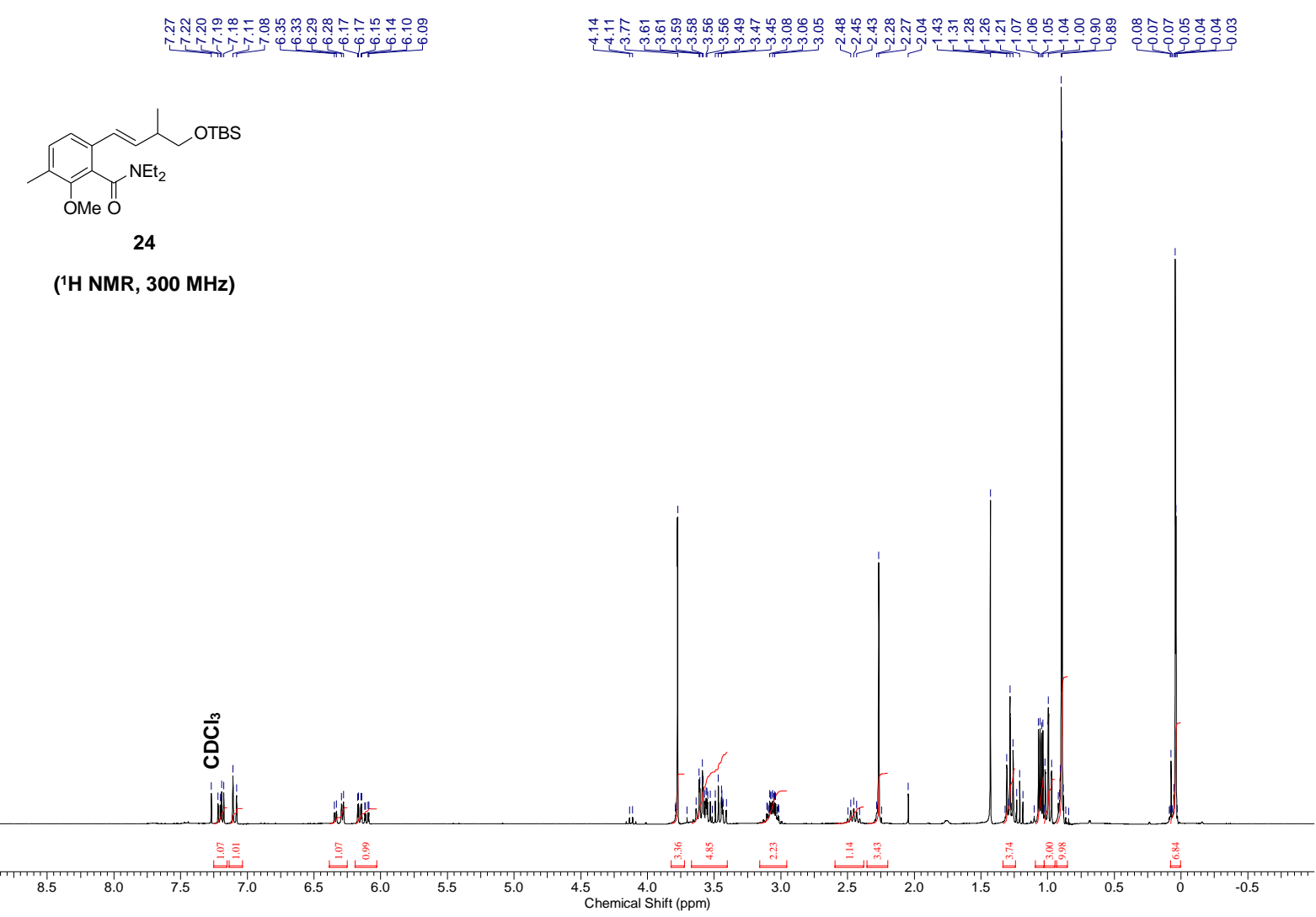

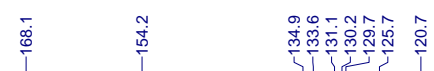

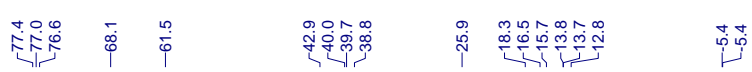

$\underbrace{\mathrm{NEt} \mathrm{O}_{2}}_{\mathrm{OMeO}}$ OTBS

ญ̊ํำ

24

$\left({ }^{13} \mathrm{C} \mathrm{NMR}, 75 \mathrm{MHz}\right)$

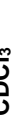

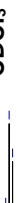

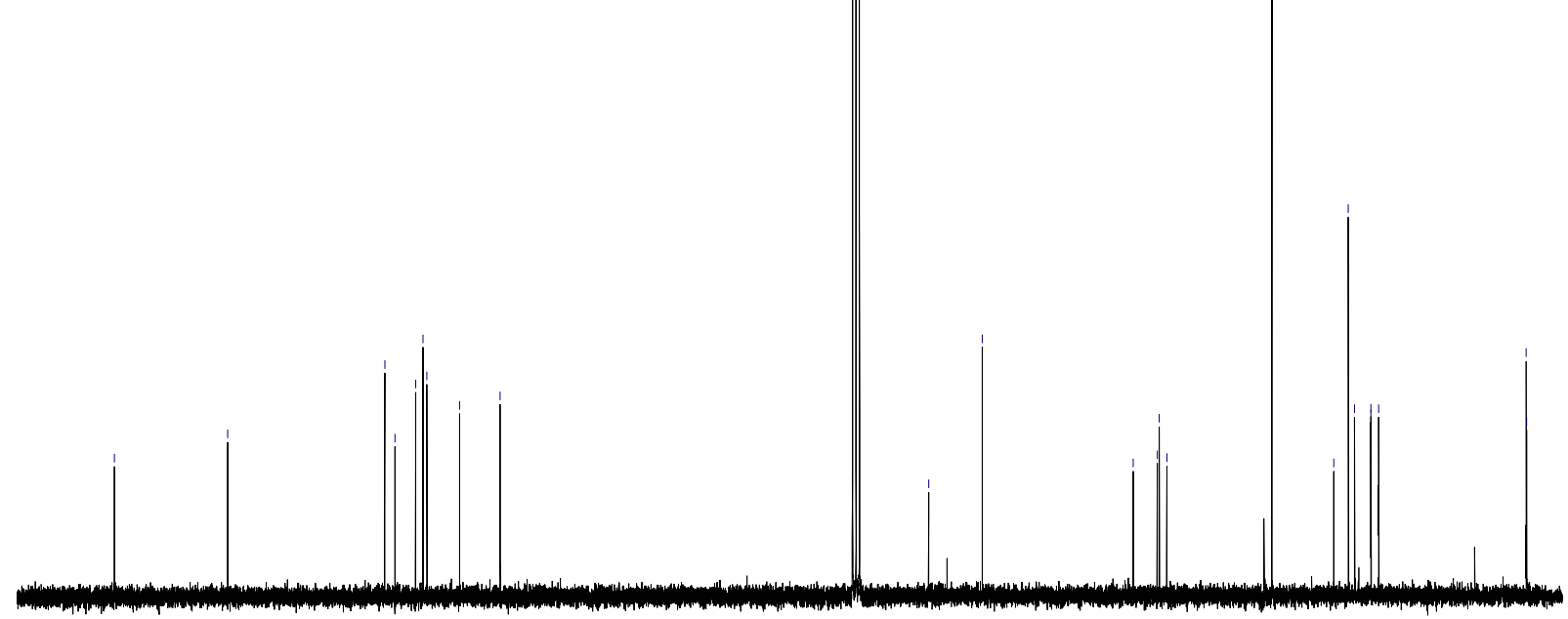

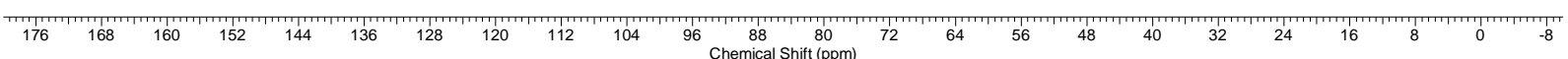




$$
\text { Eto } \overbrace{\mathrm{OH}}^{\mathrm{O}=}
$$

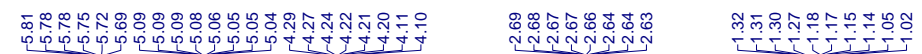

33a

('H NMR, 300 MHz)

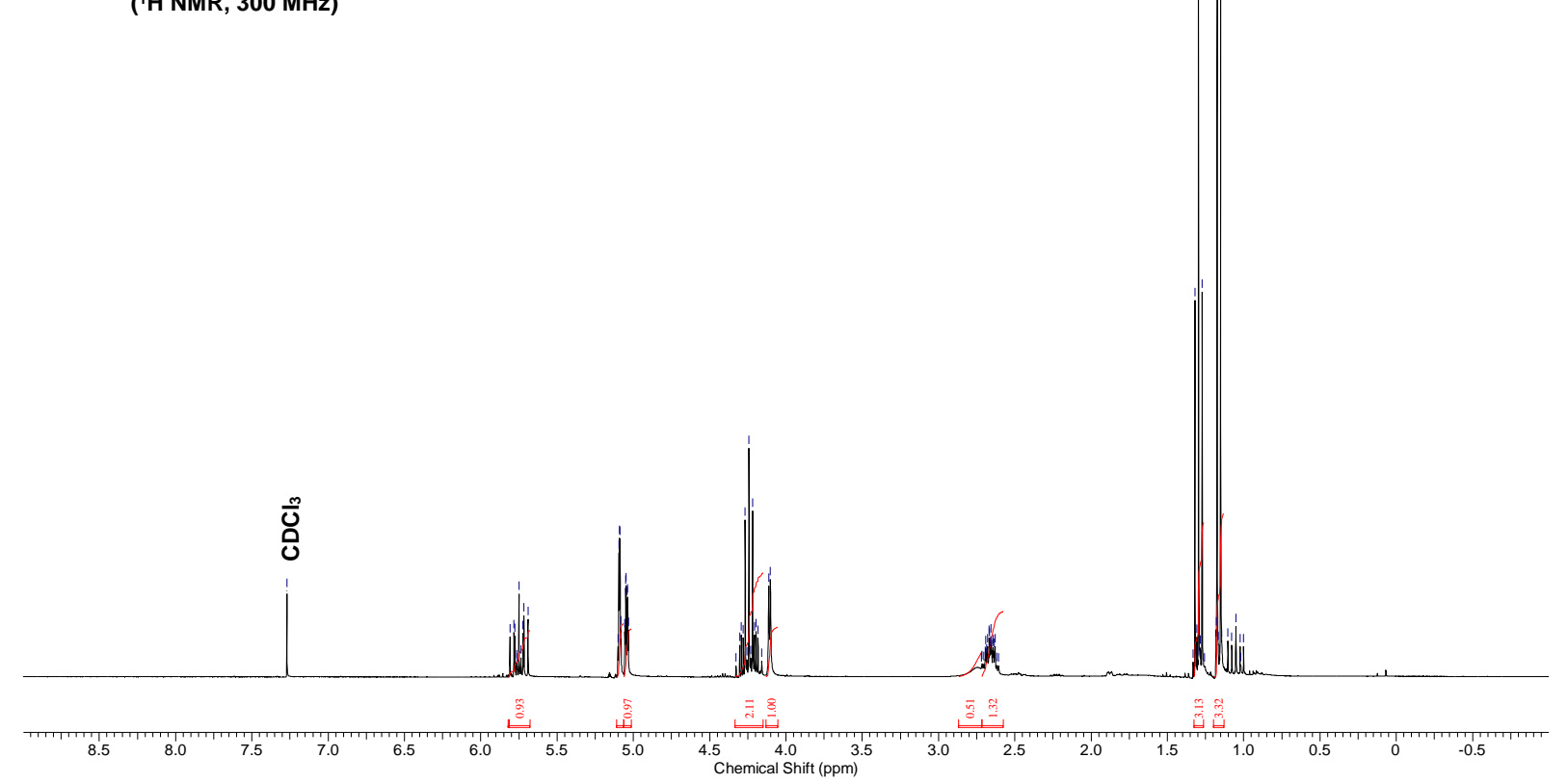

$\underset{\frac{7}{i}}{i}$<smiles>C=CC(O)C(O)CO</smiles>

$33 a$

$\left({ }^{13} \mathrm{C}\right.$ NMR, $\left.75 \mathrm{MHz}\right)$

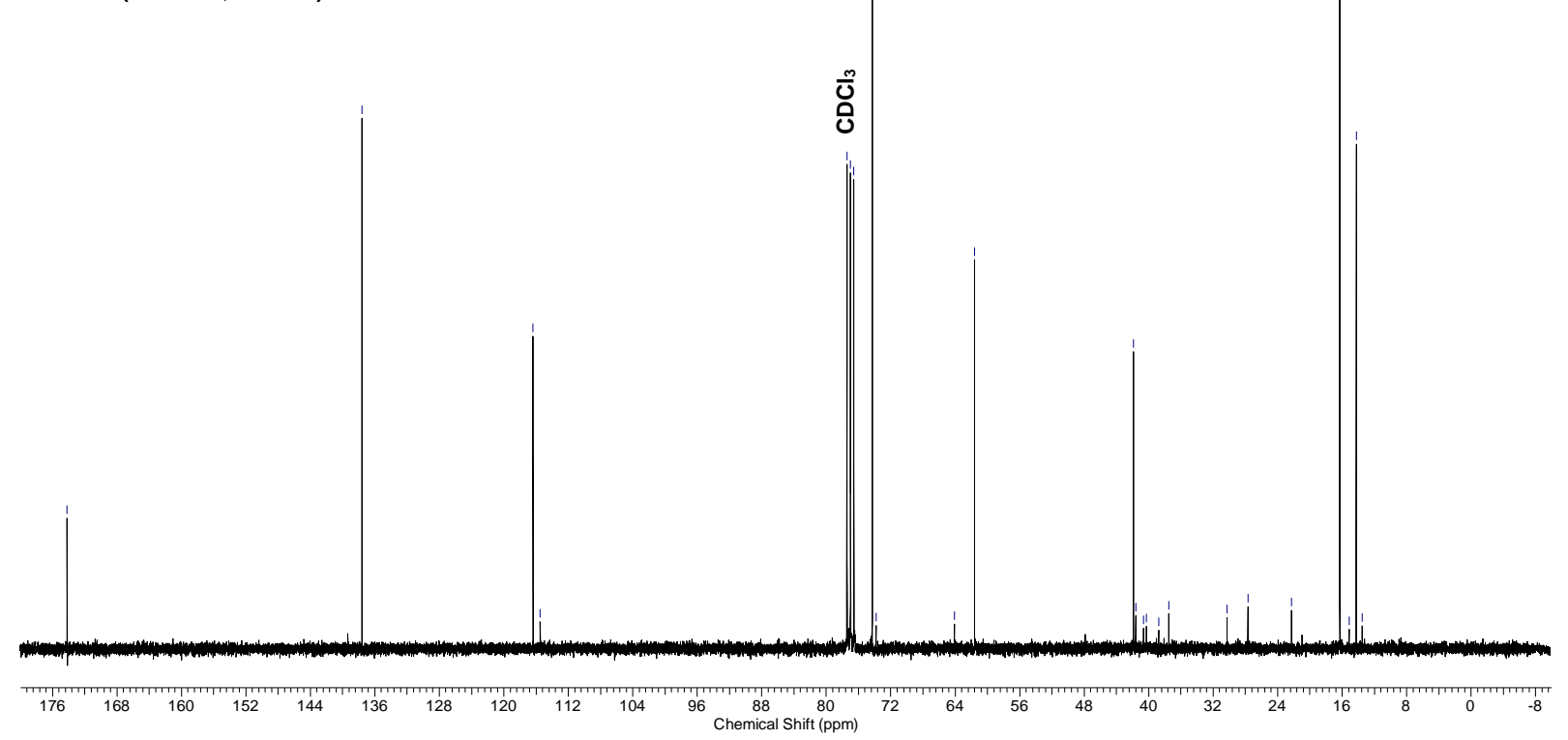




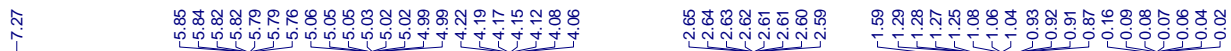<smiles>C=CCC(O)C(=O)OCC</smiles>

34

( ${ }^{1} \mathrm{H}$ NMR, $\left.300 \mathrm{MHz}\right)$

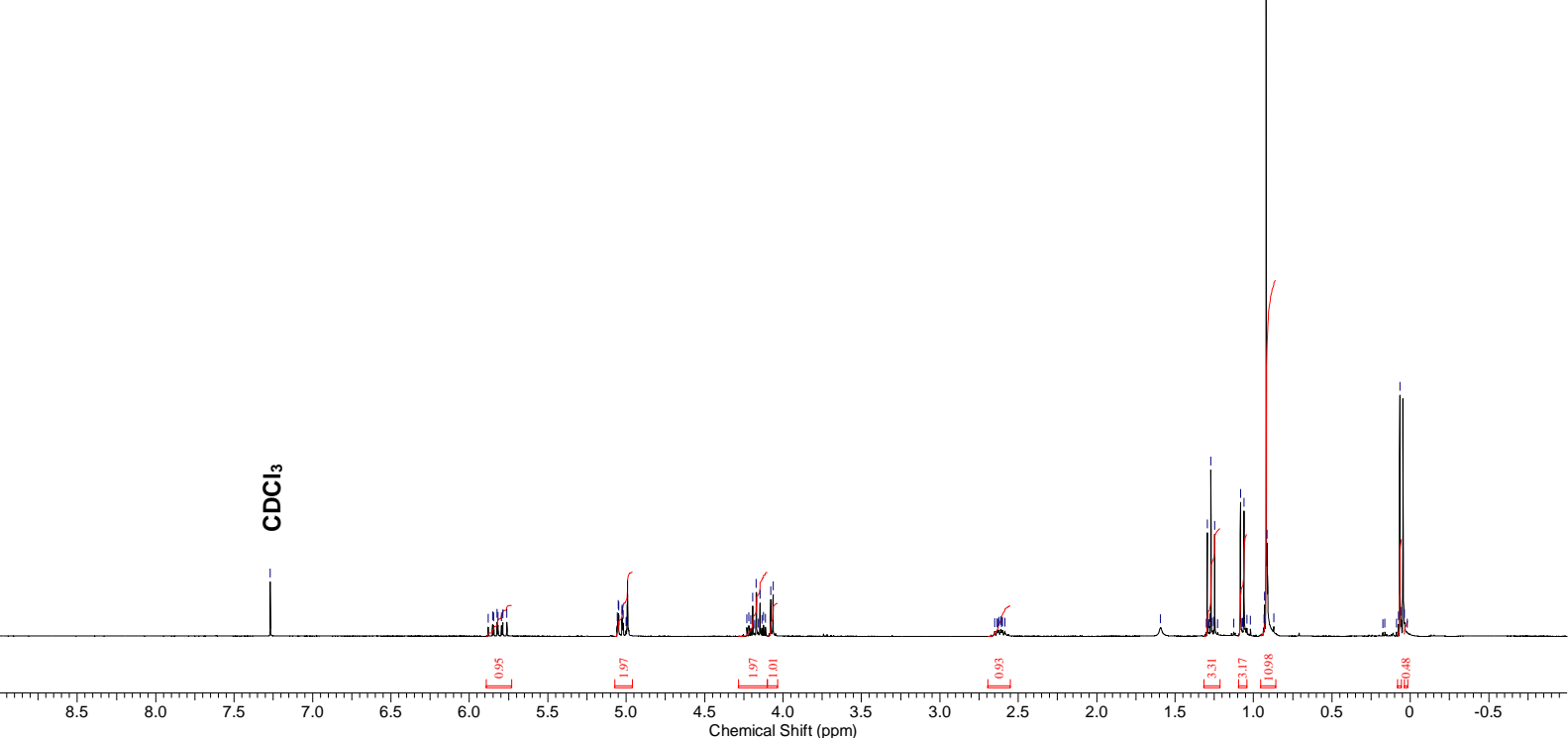

$\stackrel{\substack{i \\ \Gamma}}{\Gamma}$

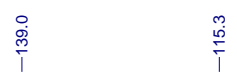<smiles>C=CCC(O)C(=O)OCC</smiles>

34

( ${ }^{13} \mathrm{C}$ NMR, $75 \mathrm{MHz}$ )

융 

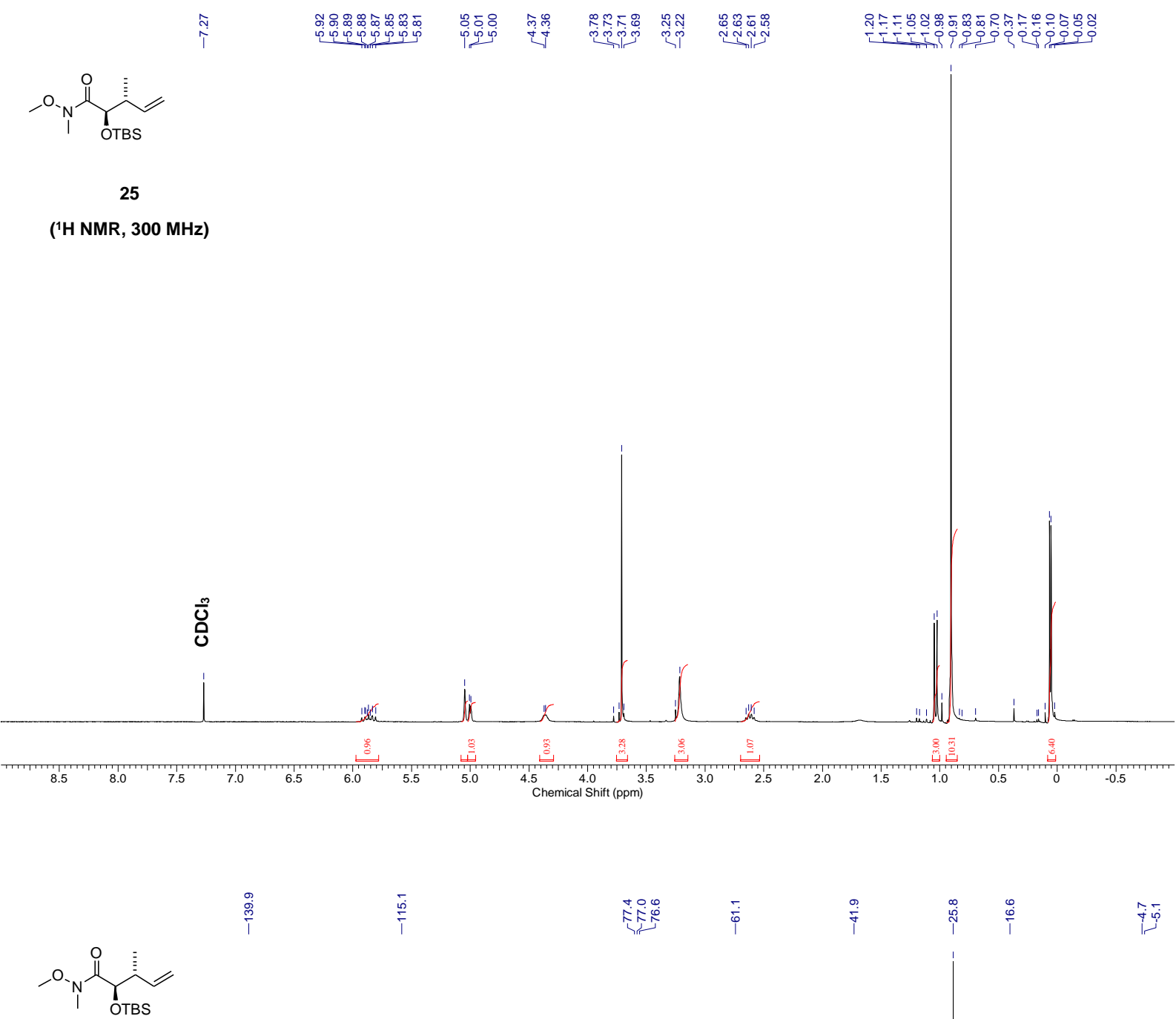

25

$\left({ }^{13} \mathrm{C}\right.$ NMR, $\left.75 \mathrm{MHz}\right)$

gิ

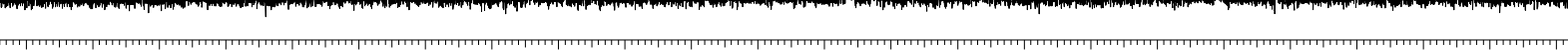

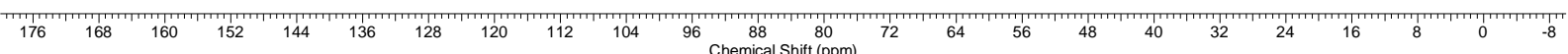


年罢

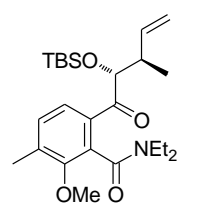

26

( ${ }^{1} \mathrm{H}$ NMR, $300 \mathrm{MHz}$ )

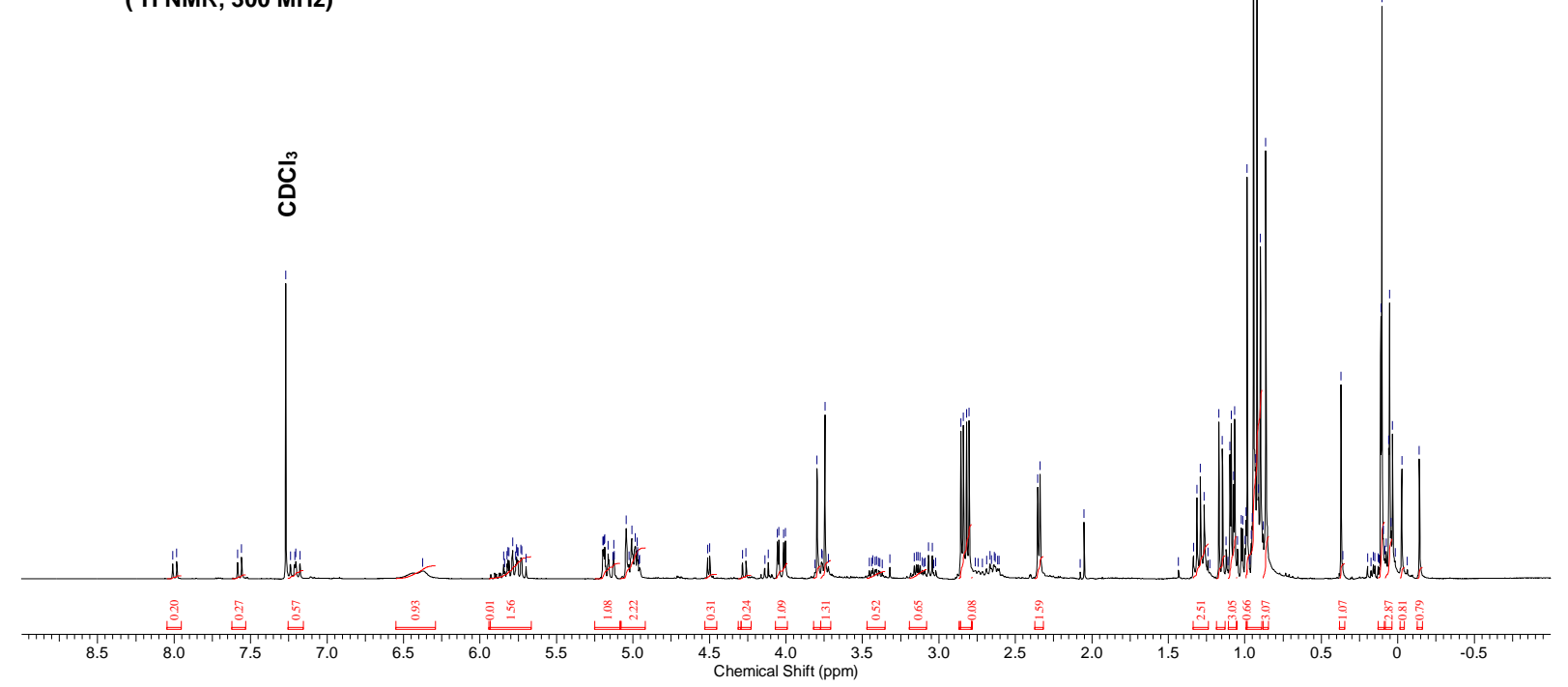

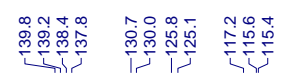

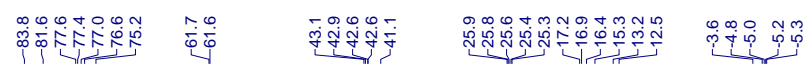

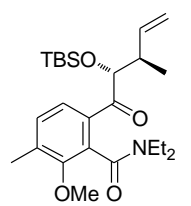

26

( ${ }^{13} \mathrm{C}$ NMR, $\left.75 \mathrm{MHz}\right)$

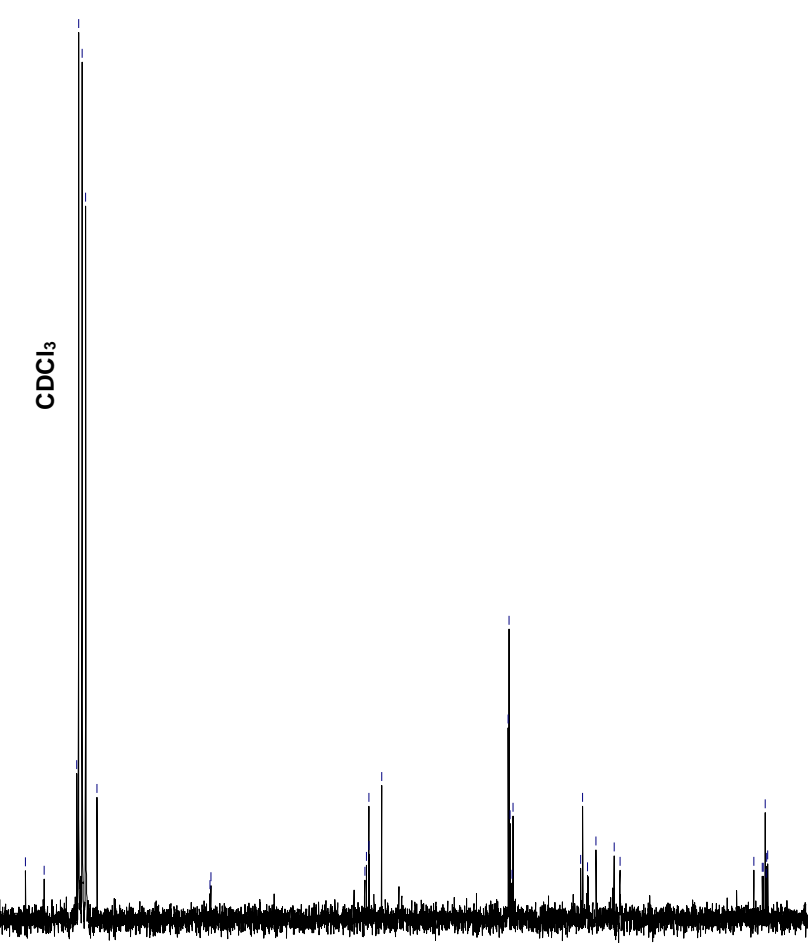

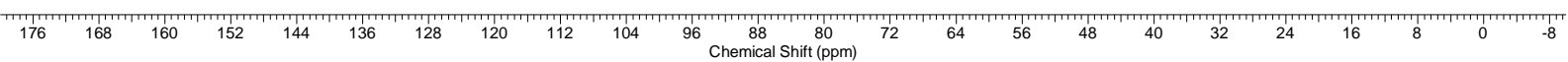




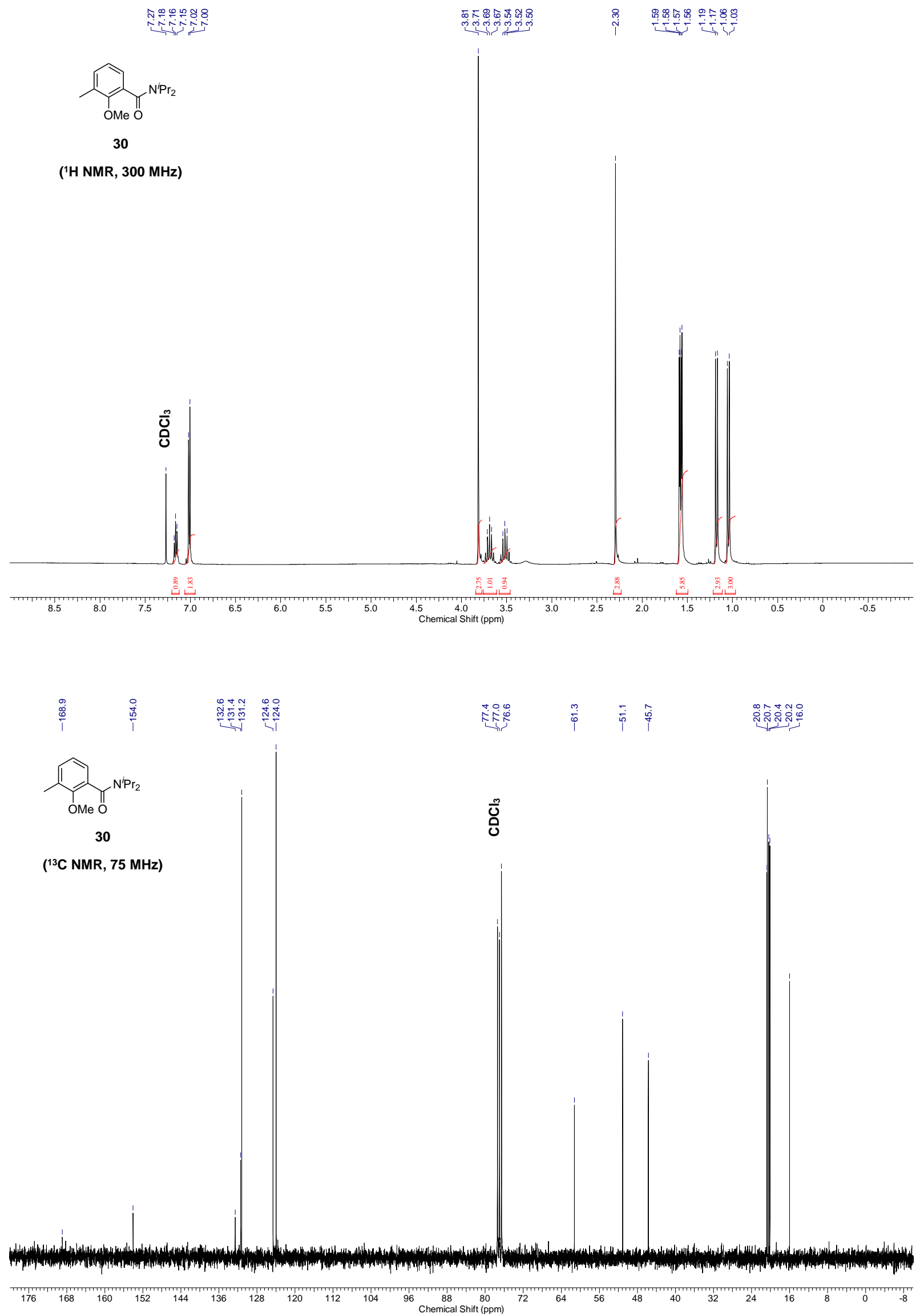




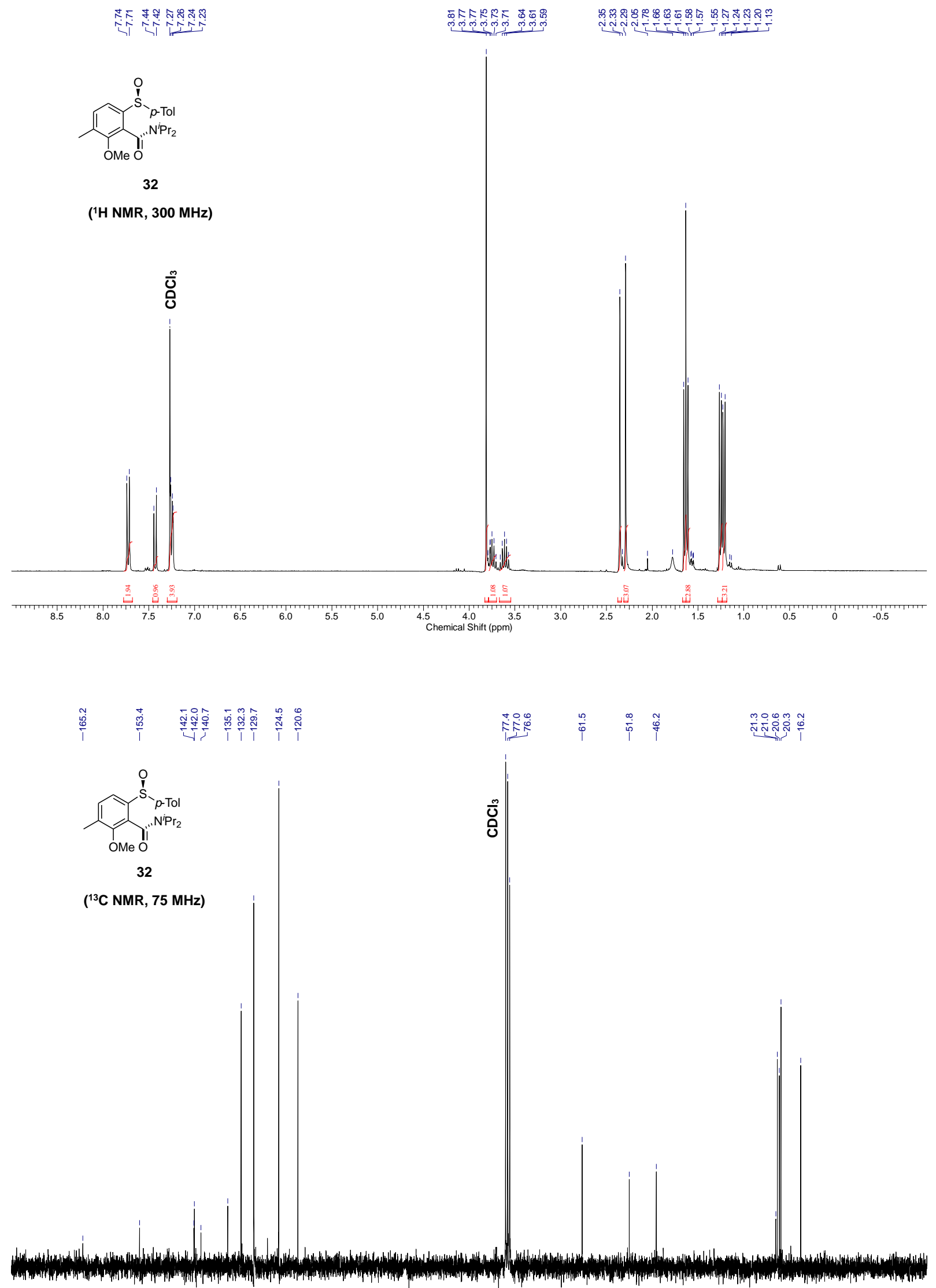

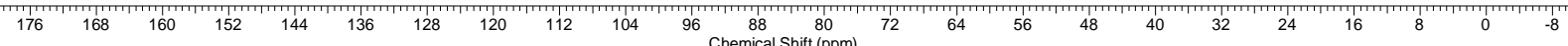




\begin{tabular}{|c|c|c|}
\hline & 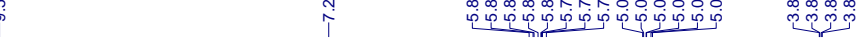 & 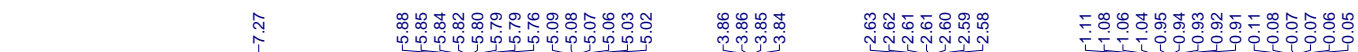 \\
\hline
\end{tabular}<smiles>C=C[CH]C([O])C=O</smiles>

35

('H NMR, $300 \mathrm{MHz}$ )

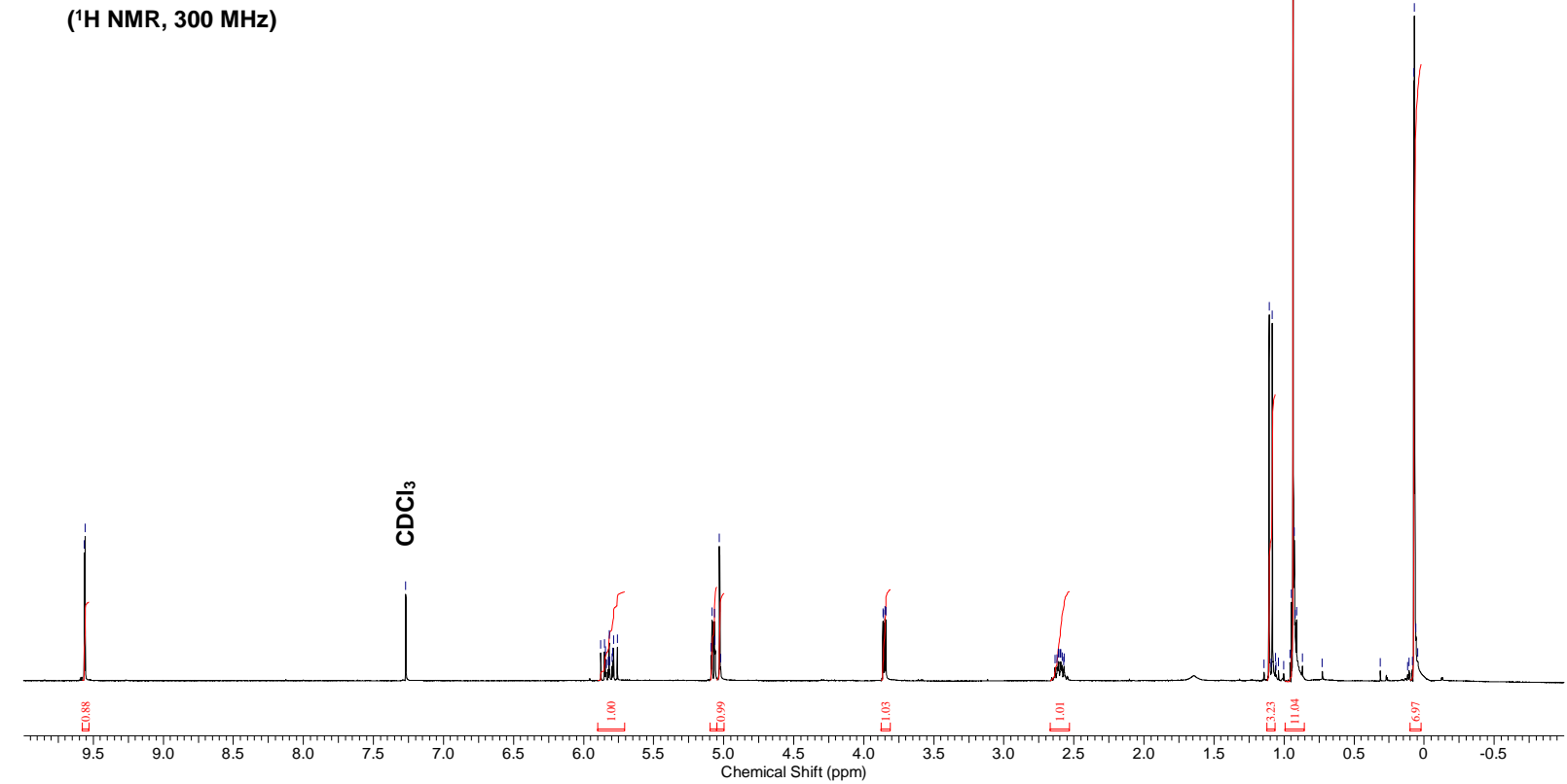

辛<smiles>C=C[C@H]([18OH])C=O</smiles> 


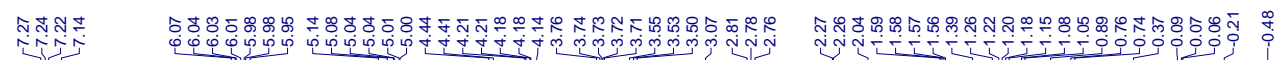

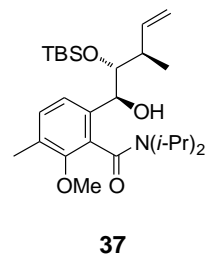

( ${ }^{1} \mathrm{H}$ NMR, $300 \mathrm{MHz}$ )

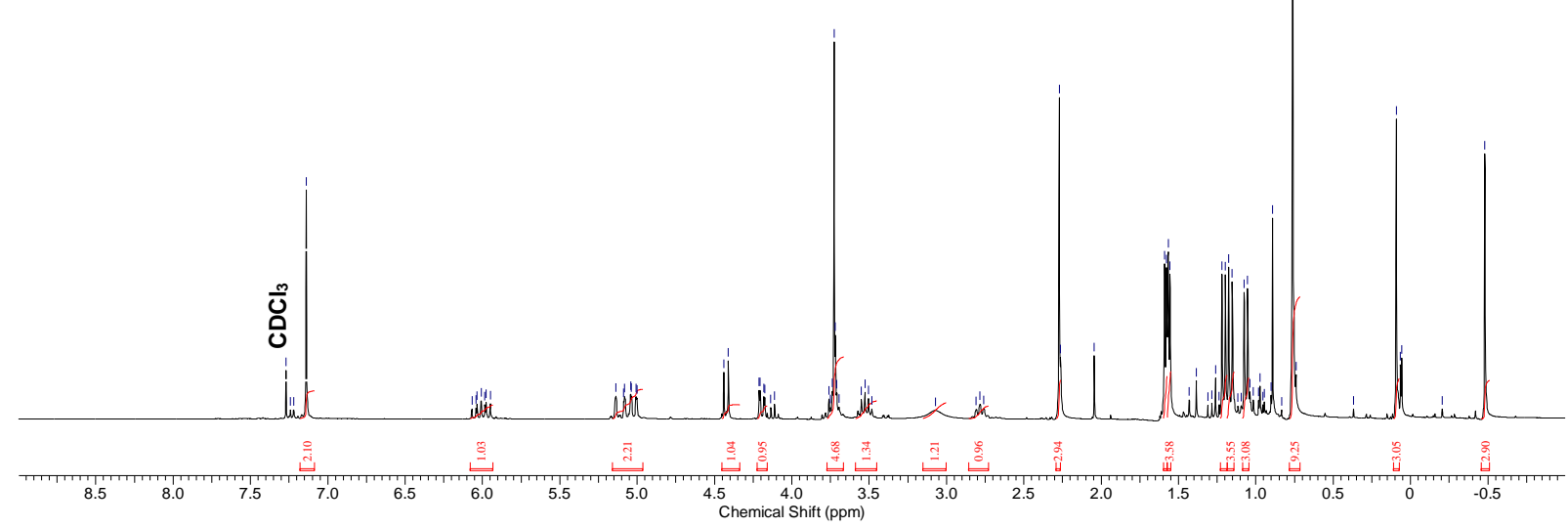

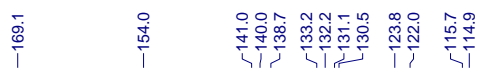

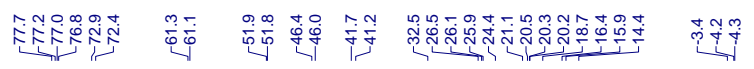

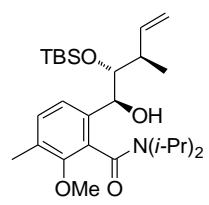

37

( ${ }^{13} \mathrm{C}$ NMR, $\left.125 \mathrm{MHz}\right)$

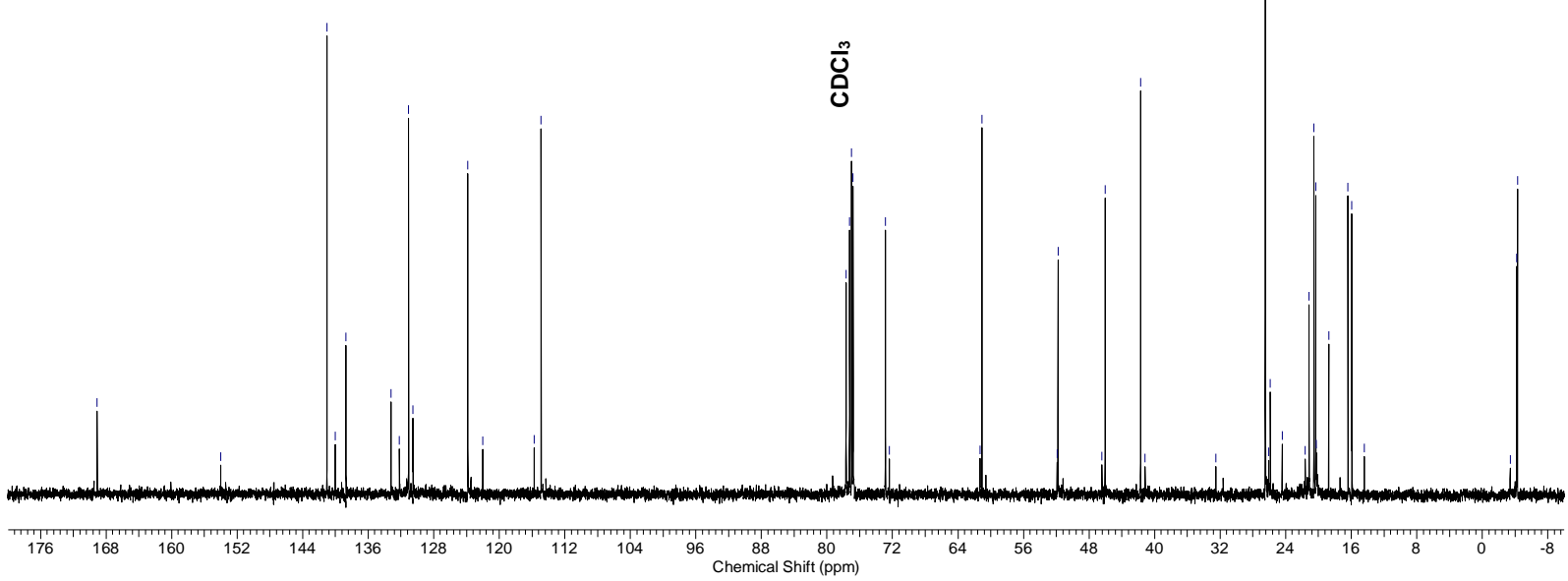




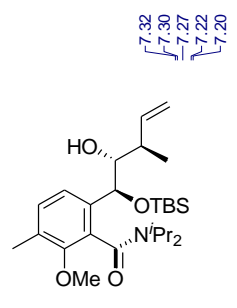

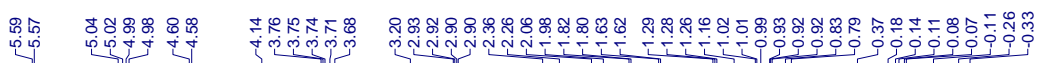

40

('H NMR, $600 \mathrm{MHz}$ )

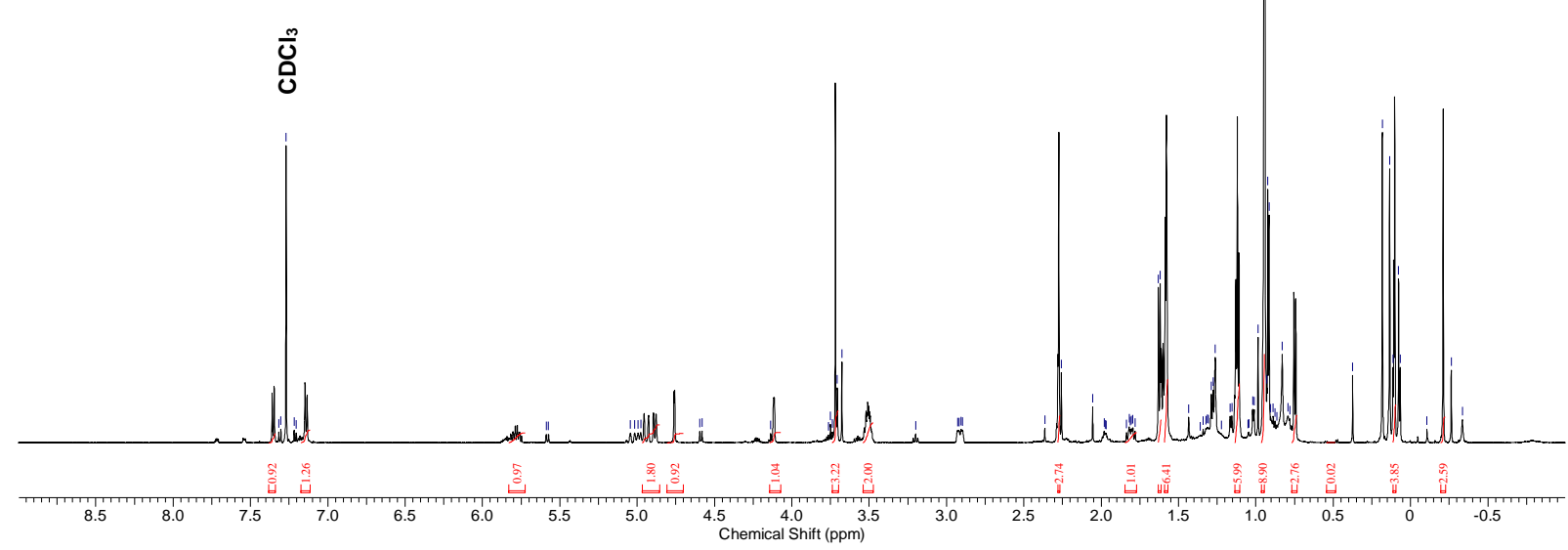

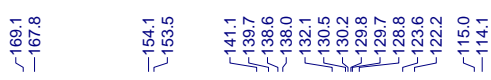<smiles>C=CC(C)C(O)C(c1ccc(C)c(OC)c1C(=O)OC)C(O)C(=O)OC</smiles>

40

( ${ }^{13} \mathrm{C}$ NMR, $\left.150 \mathrm{MHz}\right)$

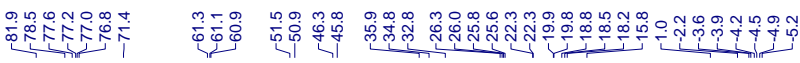

윰 
$\stackrel{\infty}{\infty}$

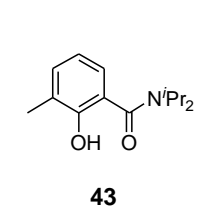

('H NMR, 300 MHz)

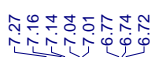

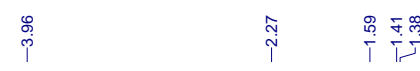

(

(1)

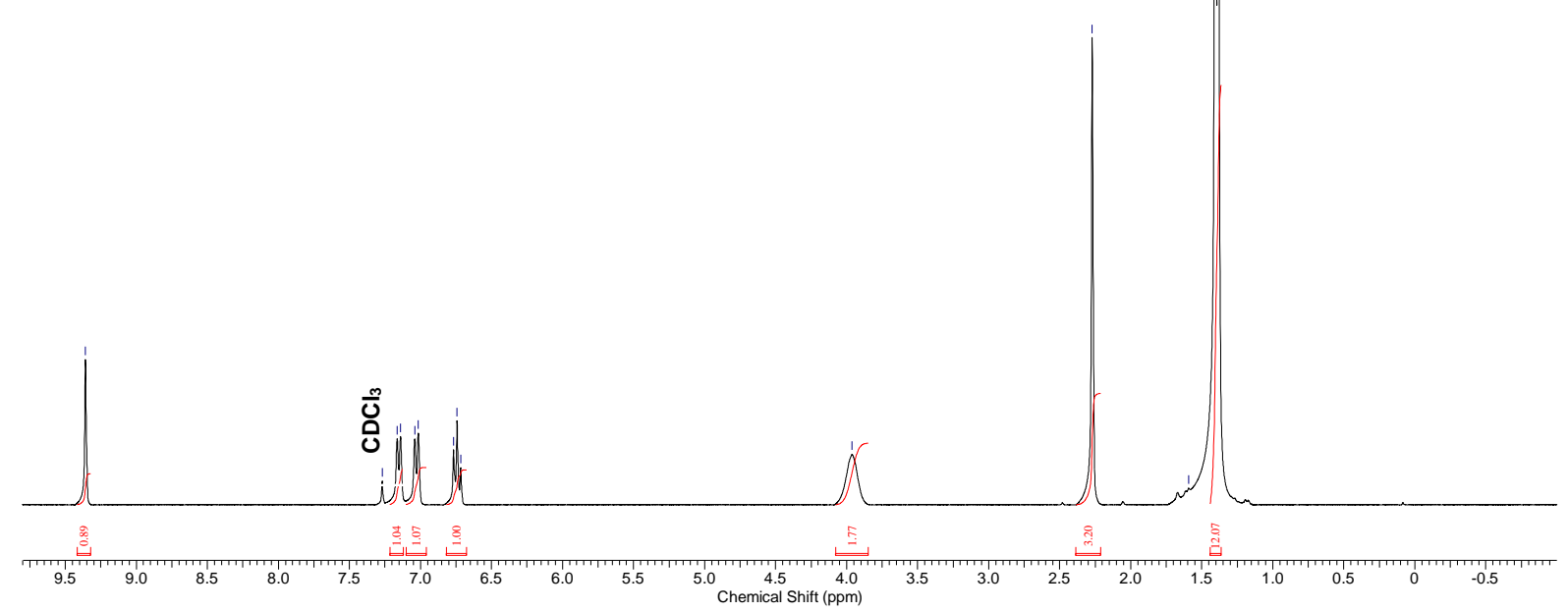<smiles>C1CCC(C2CCCC2)C1</smiles>

43

( ${ }^{13} \mathrm{C}$ NMR, $75 \mathrm{MHz}$ )

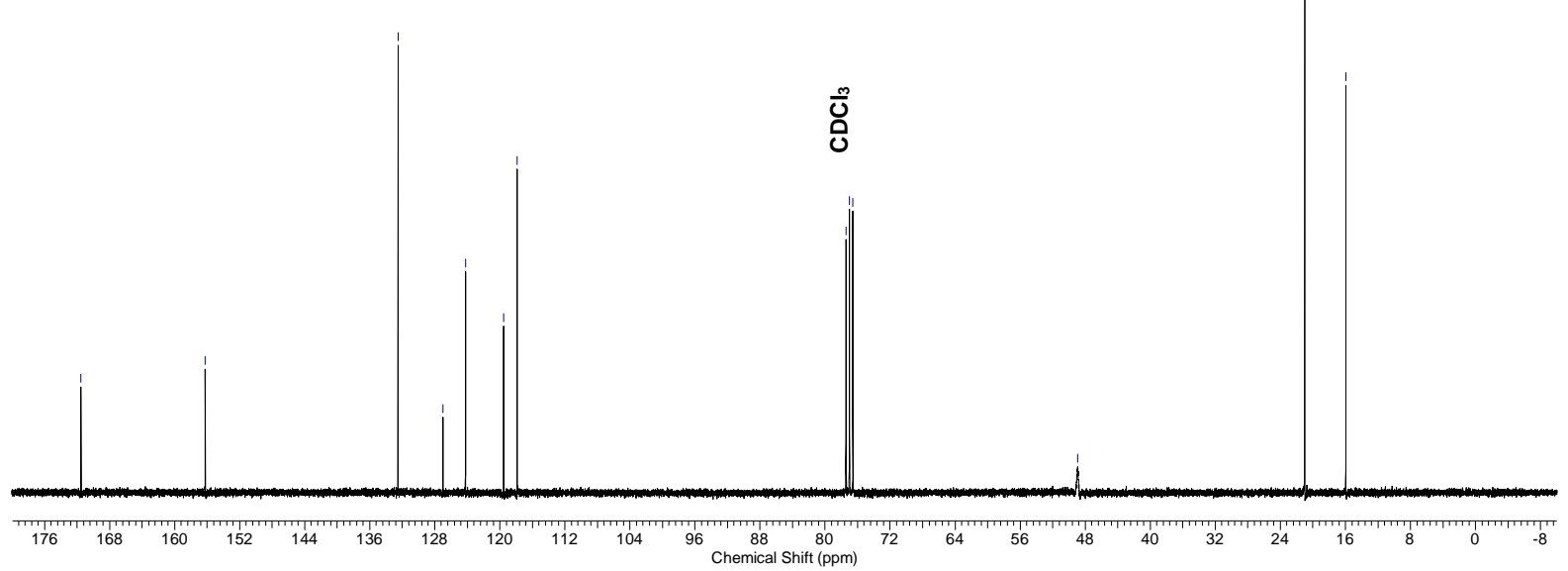



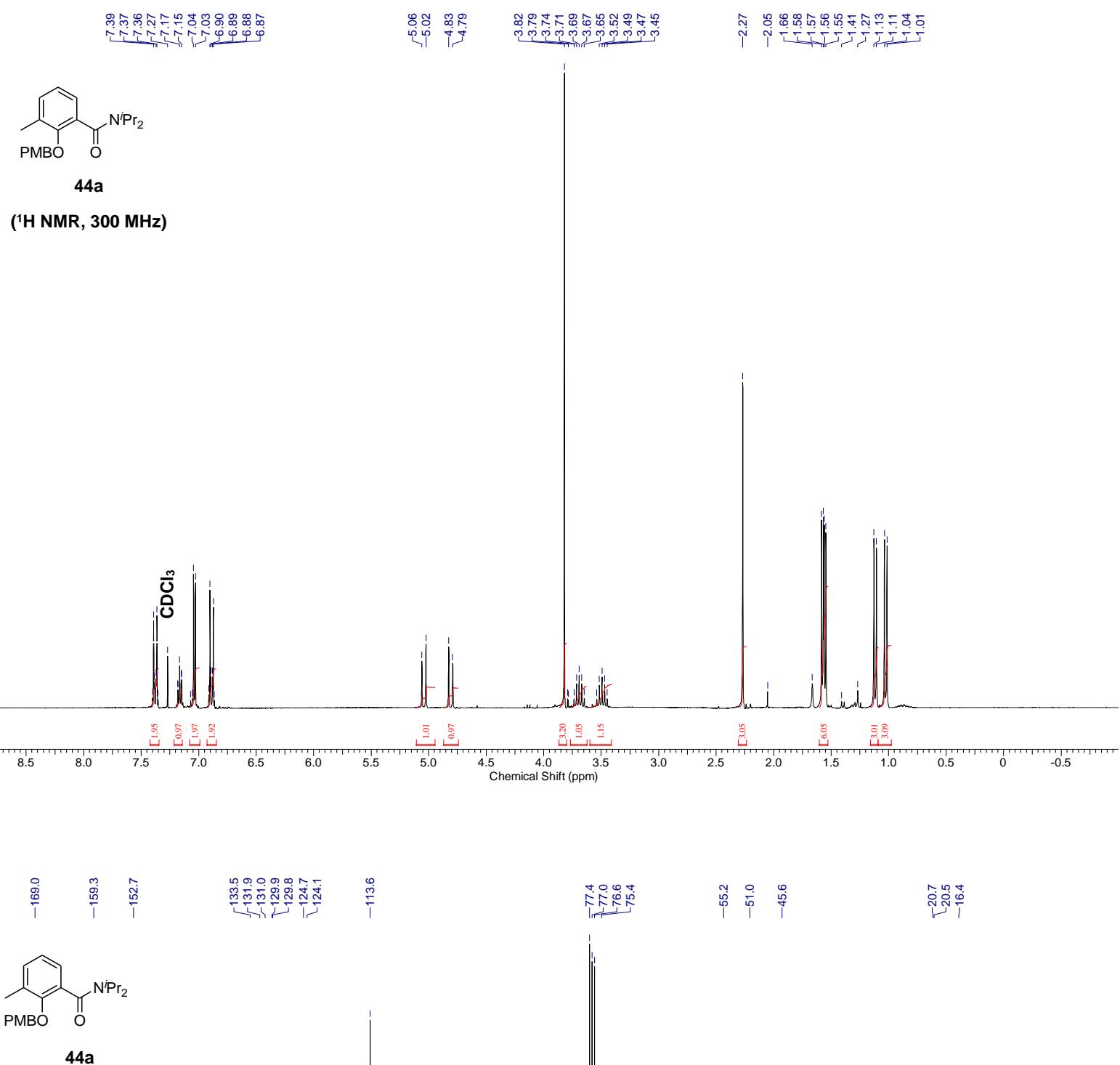

( ${ }^{13} \mathrm{C}$ NMR, $75 \mathrm{MHz}$ )

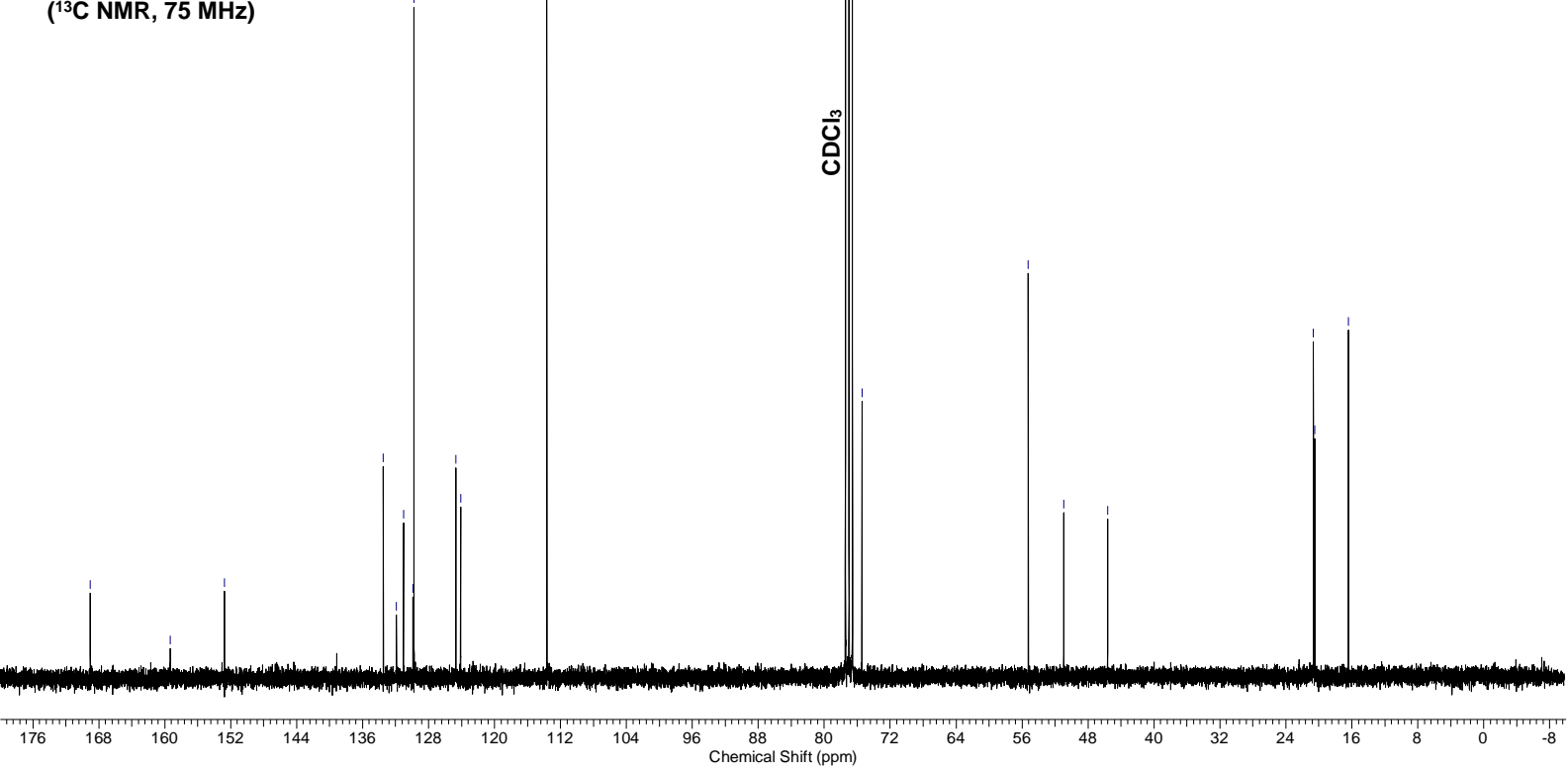




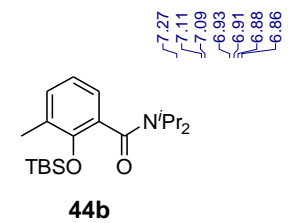

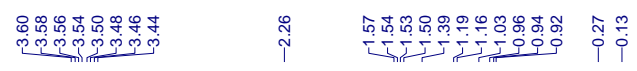

('H NMR, 300 MHz)
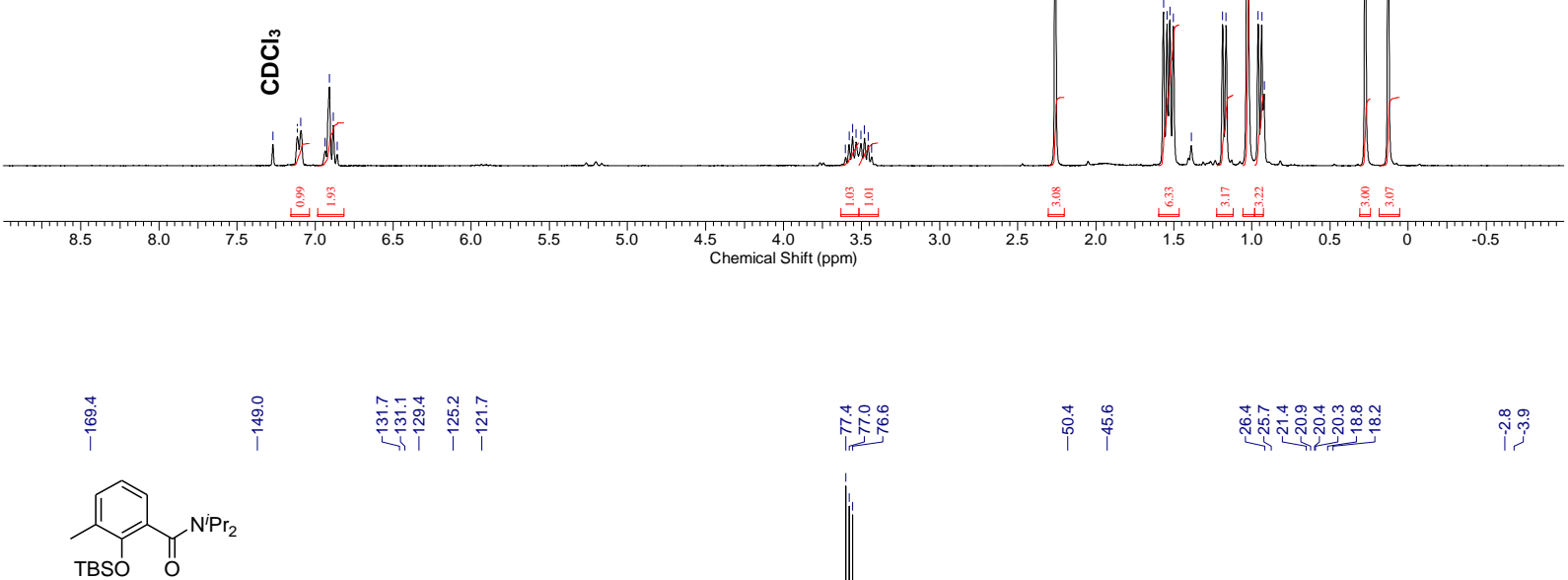

44b

( ${ }^{13} \mathrm{C}$ NMR, $75 \mathrm{MHz}$ )

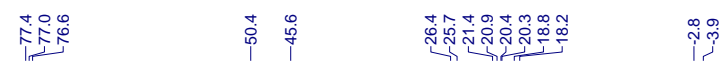

రัญ

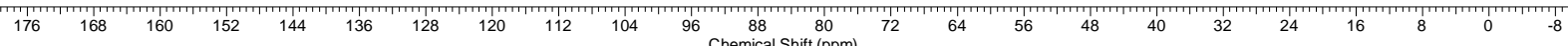




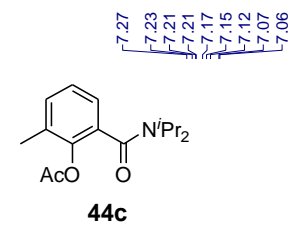

نำ

('H NMR, 300 MHz)
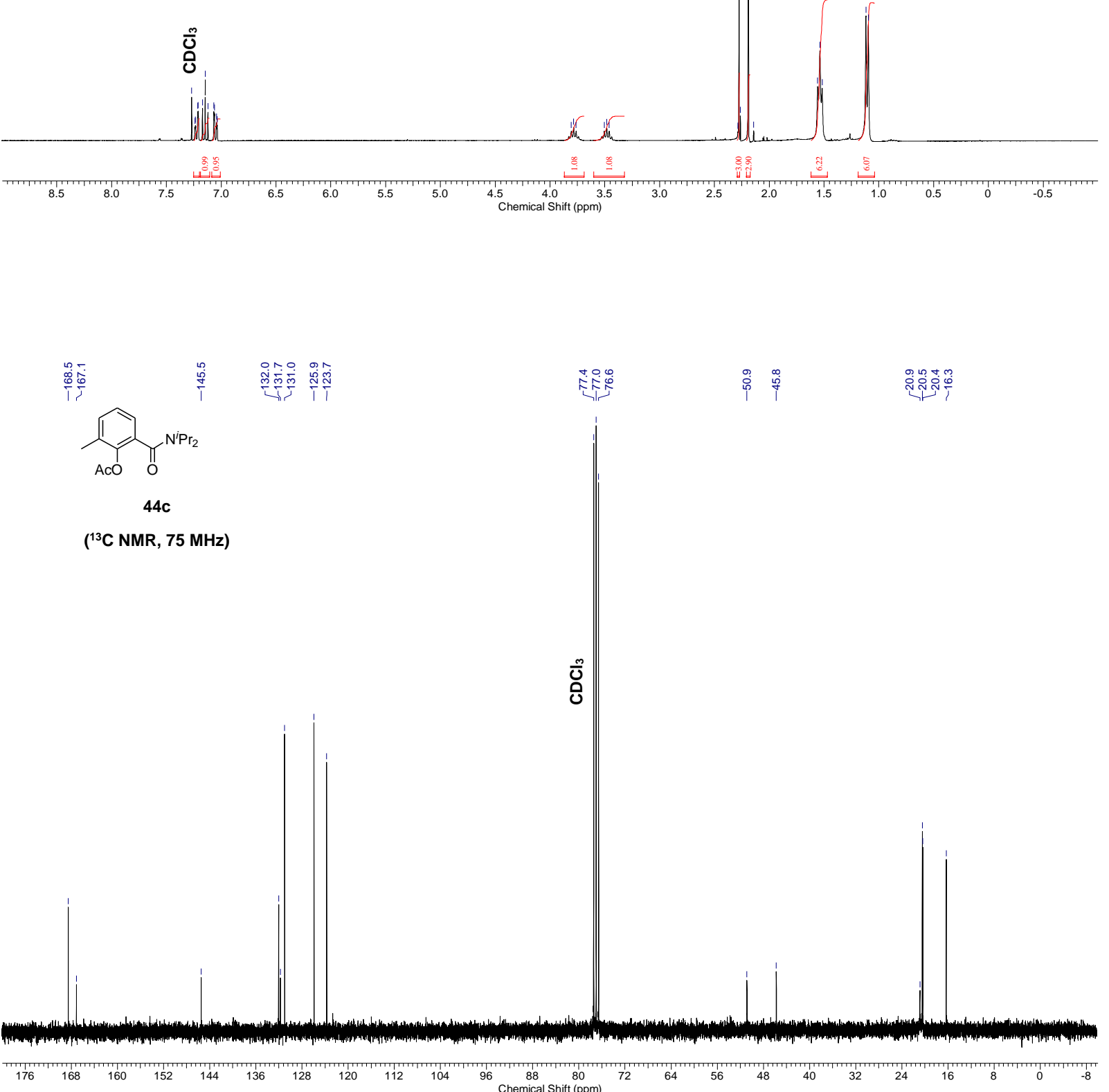


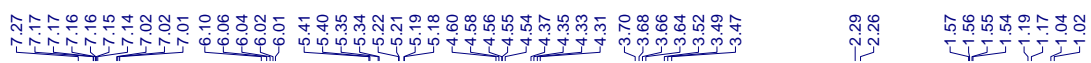

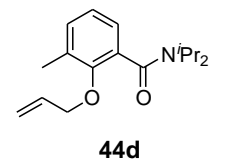

('H NMR, $300 \mathrm{MHz}$ )

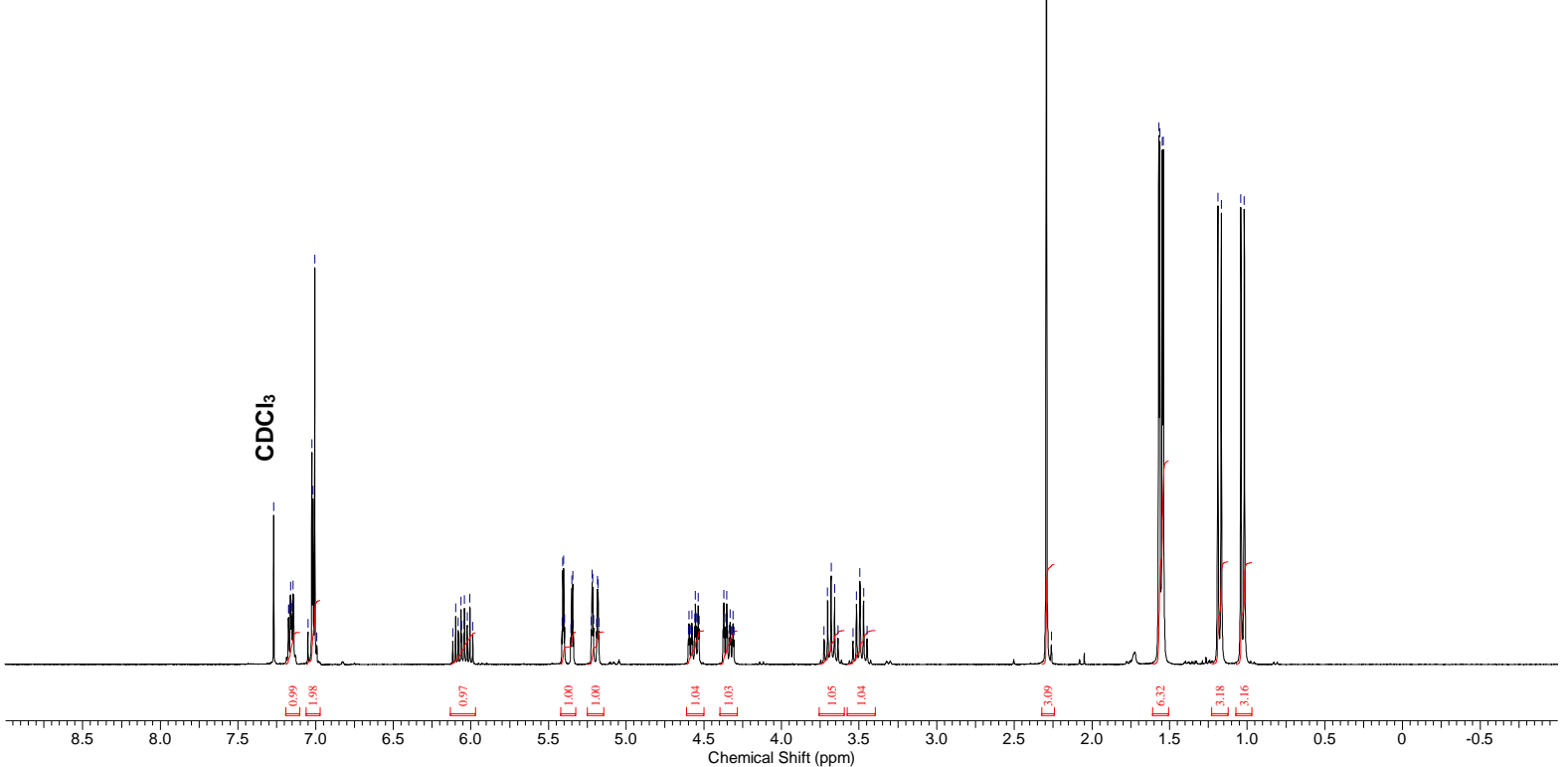

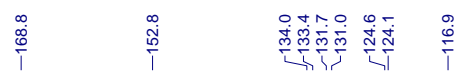

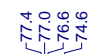

号号

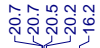

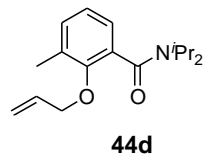

( ${ }^{13} \mathrm{C}$ NMR, $\left.75 \mathrm{MHz}\right)$

윰

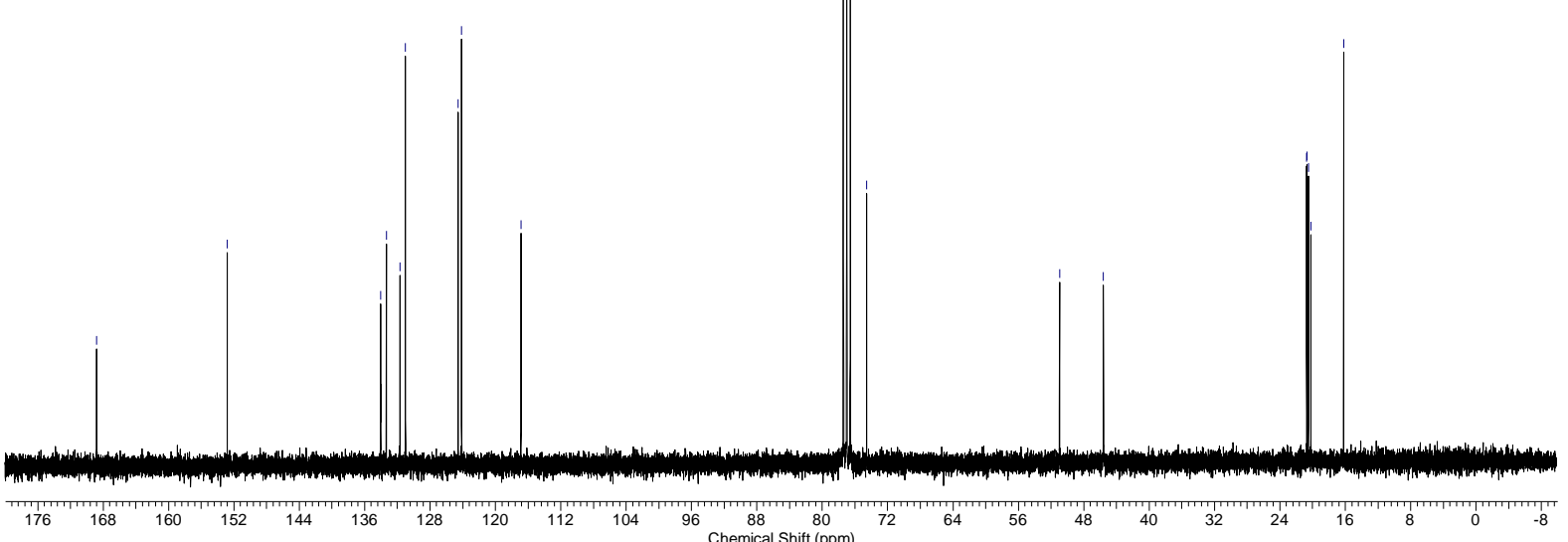




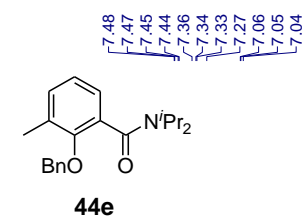

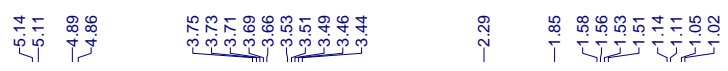

('H NMR, 300 MHz)
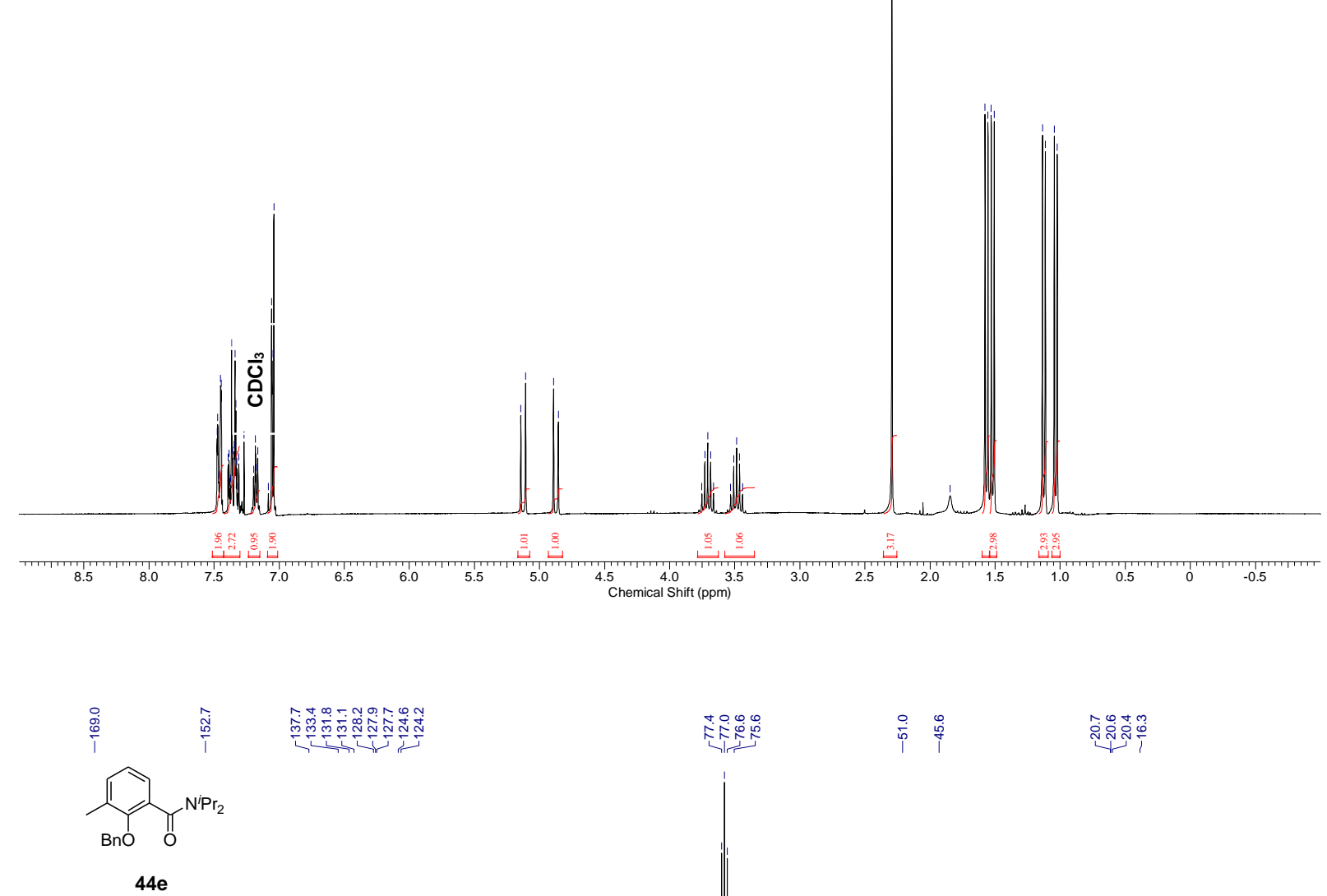

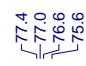

号品

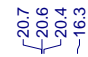

( ${ }^{13} \mathrm{C}$ NMR, $75 \mathrm{MHz}$ )

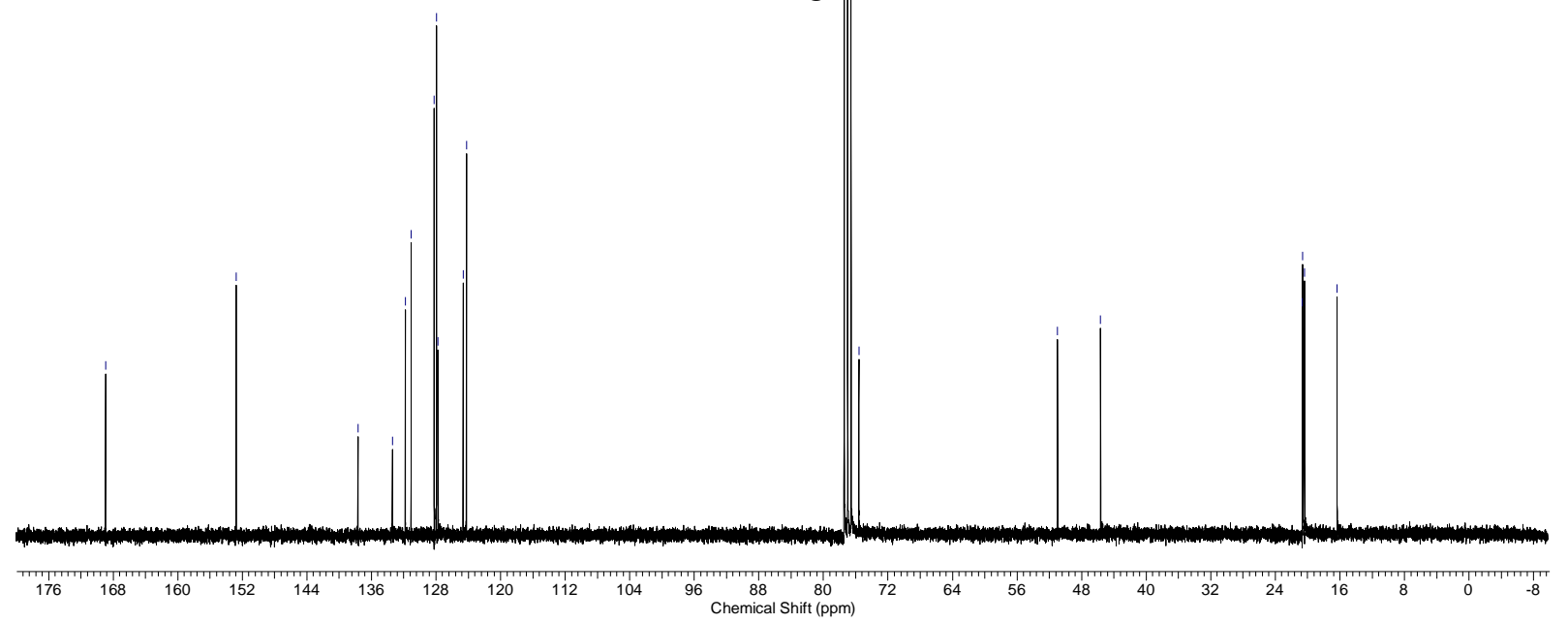




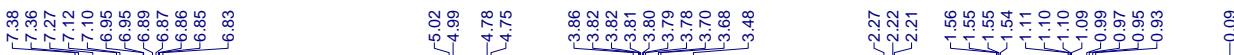

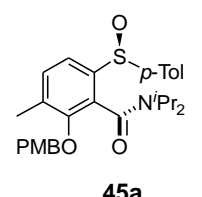

('H NMR, $300 \mathrm{MHz}$ )

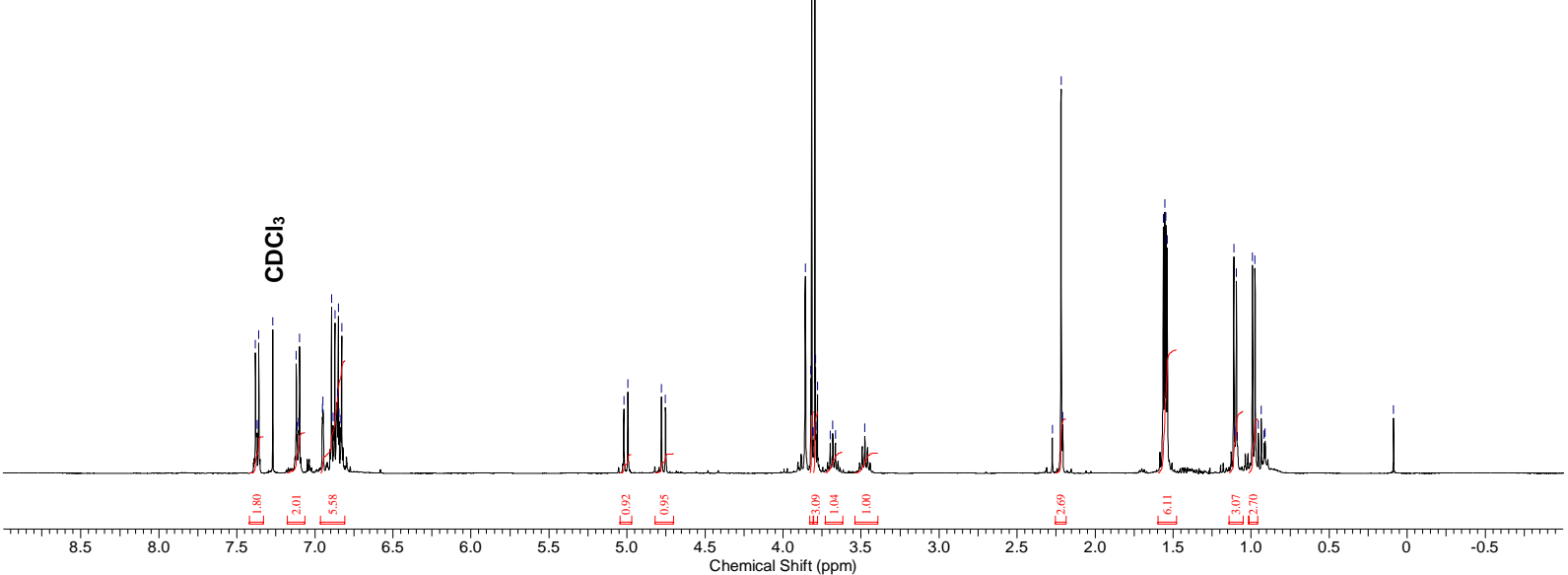

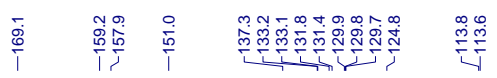

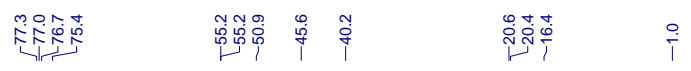

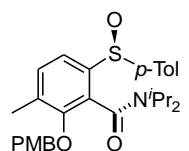

$45 a$

( ${ }^{13} \mathrm{C}$ NMR, $\left.101 \mathrm{MHz}\right)$

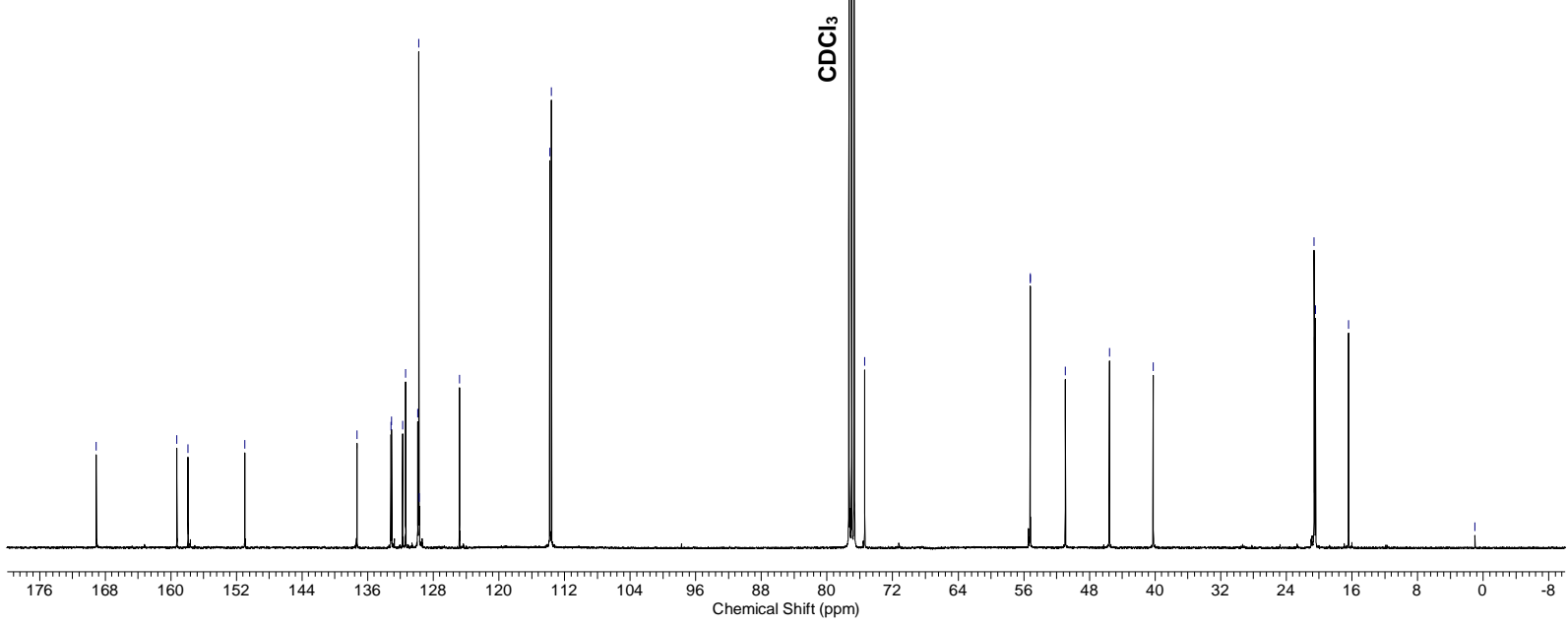




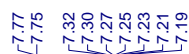

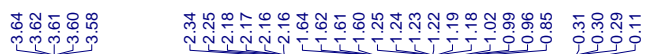

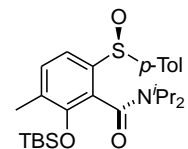

$45 \mathrm{~b}$

('H NMR, $300 \mathrm{MHz}$ )

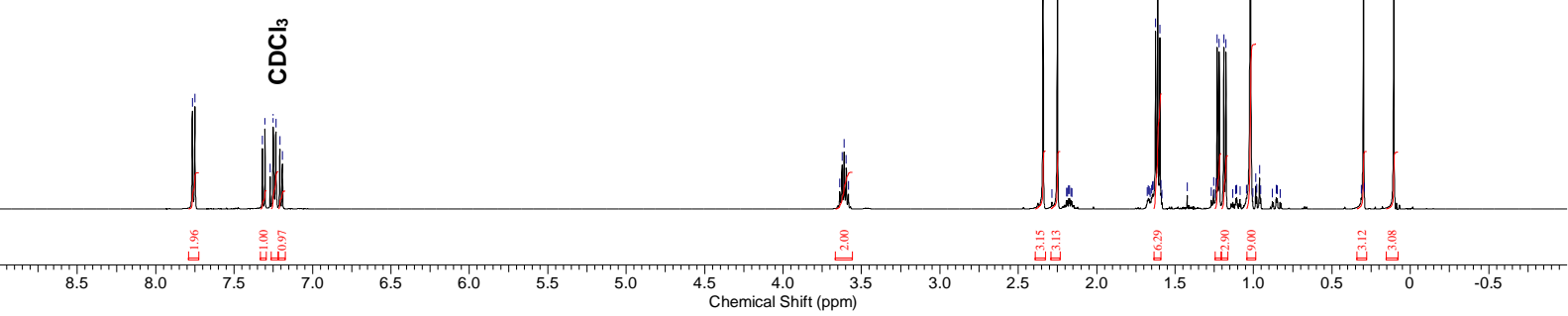

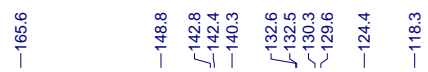

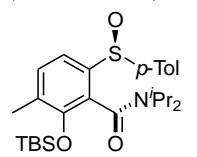

$45 \mathrm{~b}$

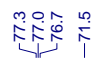

$\stackrel{m}{\substack{1 \\ i}}$

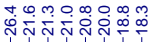

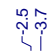

( ${ }^{13} \mathrm{C}$ NMR, $\left.75 \mathrm{MHz}\right)$

迎

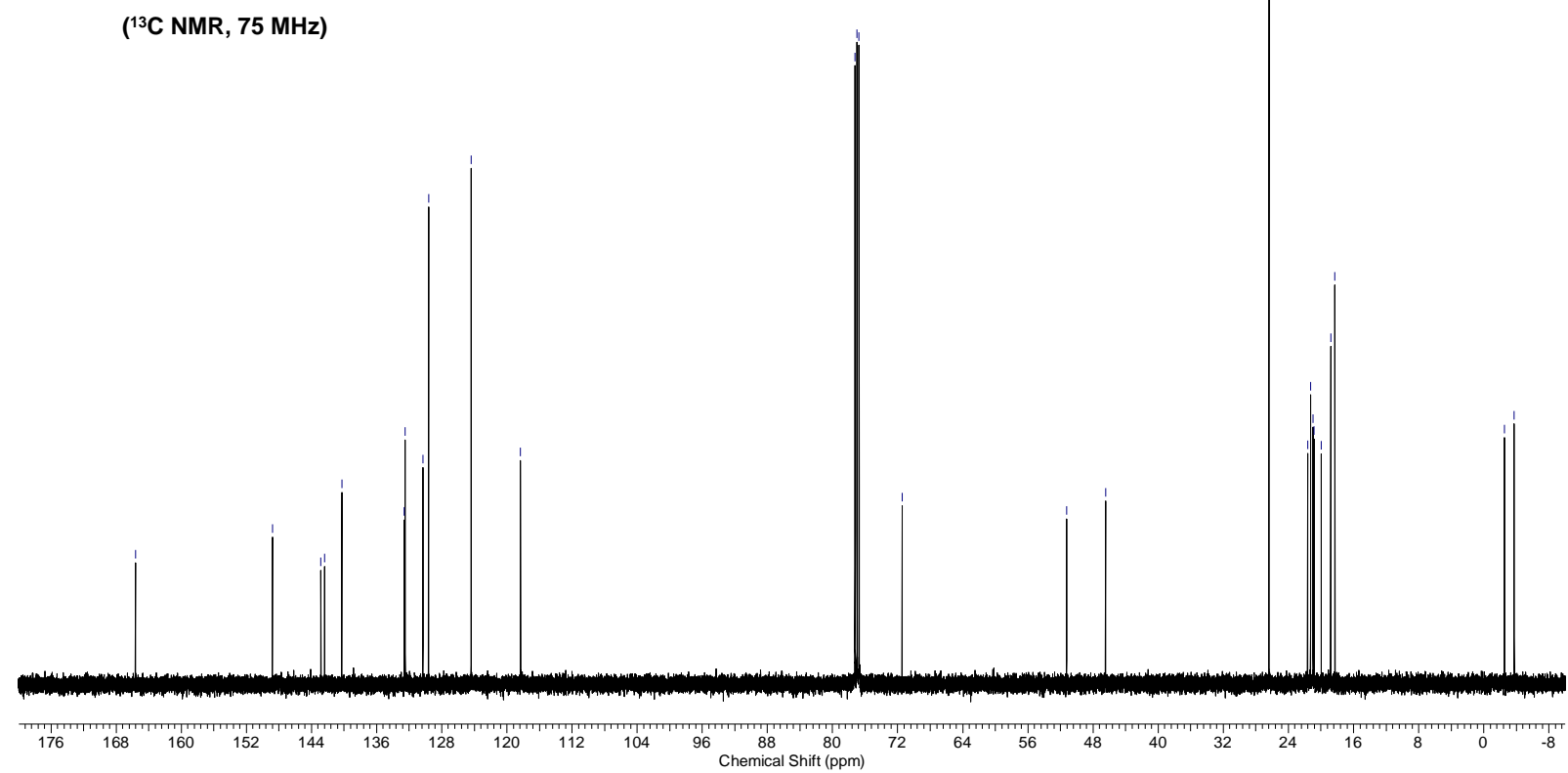




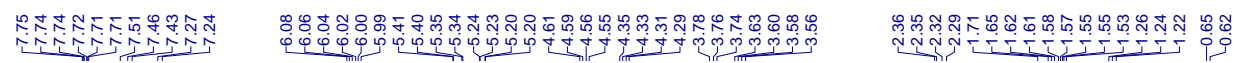

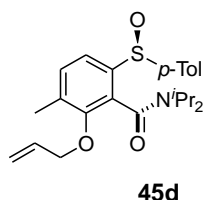

('H NMR, $300 \mathrm{MHz}$ )

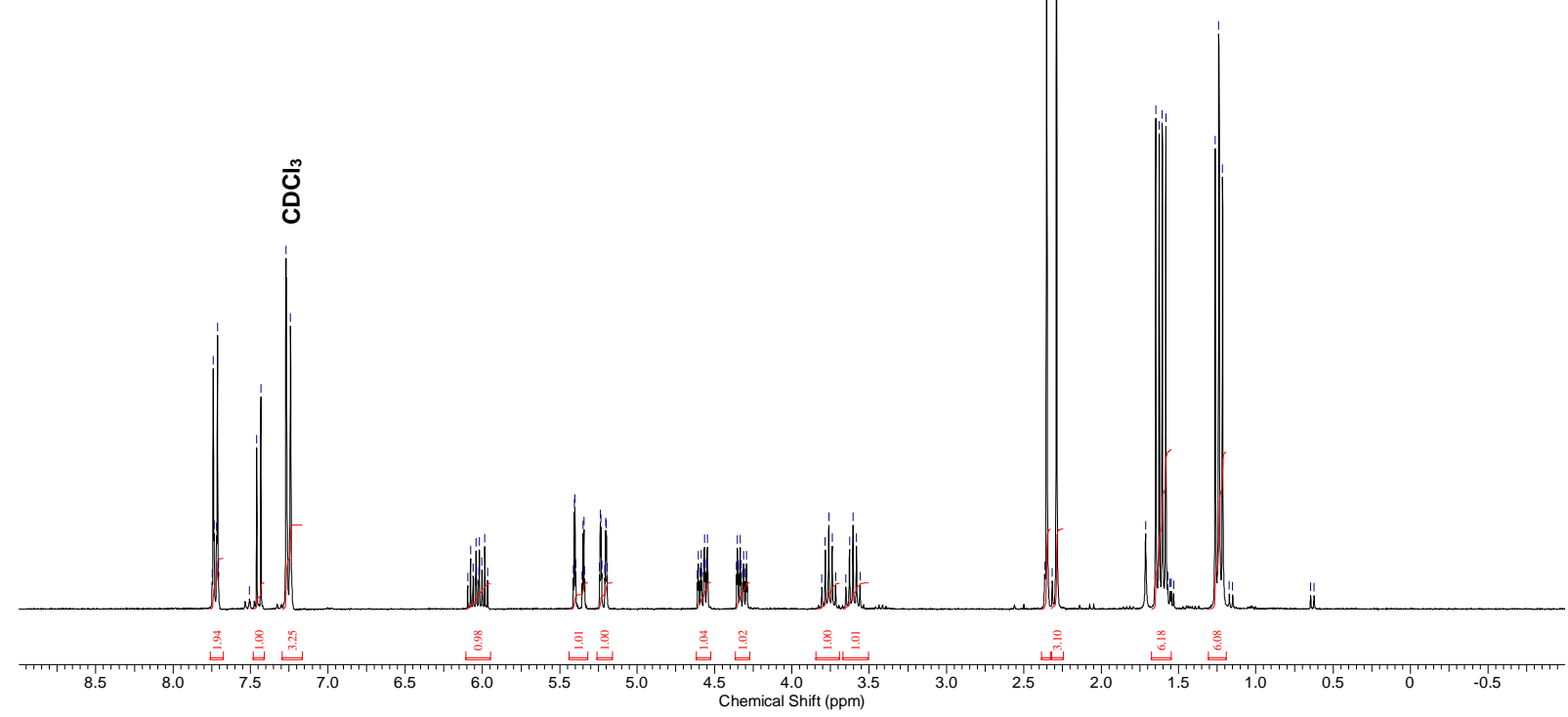

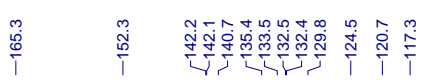

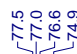

$\stackrel{\infty}{\bar{p}}$

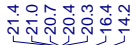

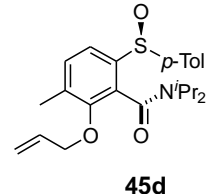

45d

( ${ }^{13} \mathrm{C}$ NMR, $\left.75 \mathrm{MHz}\right)$

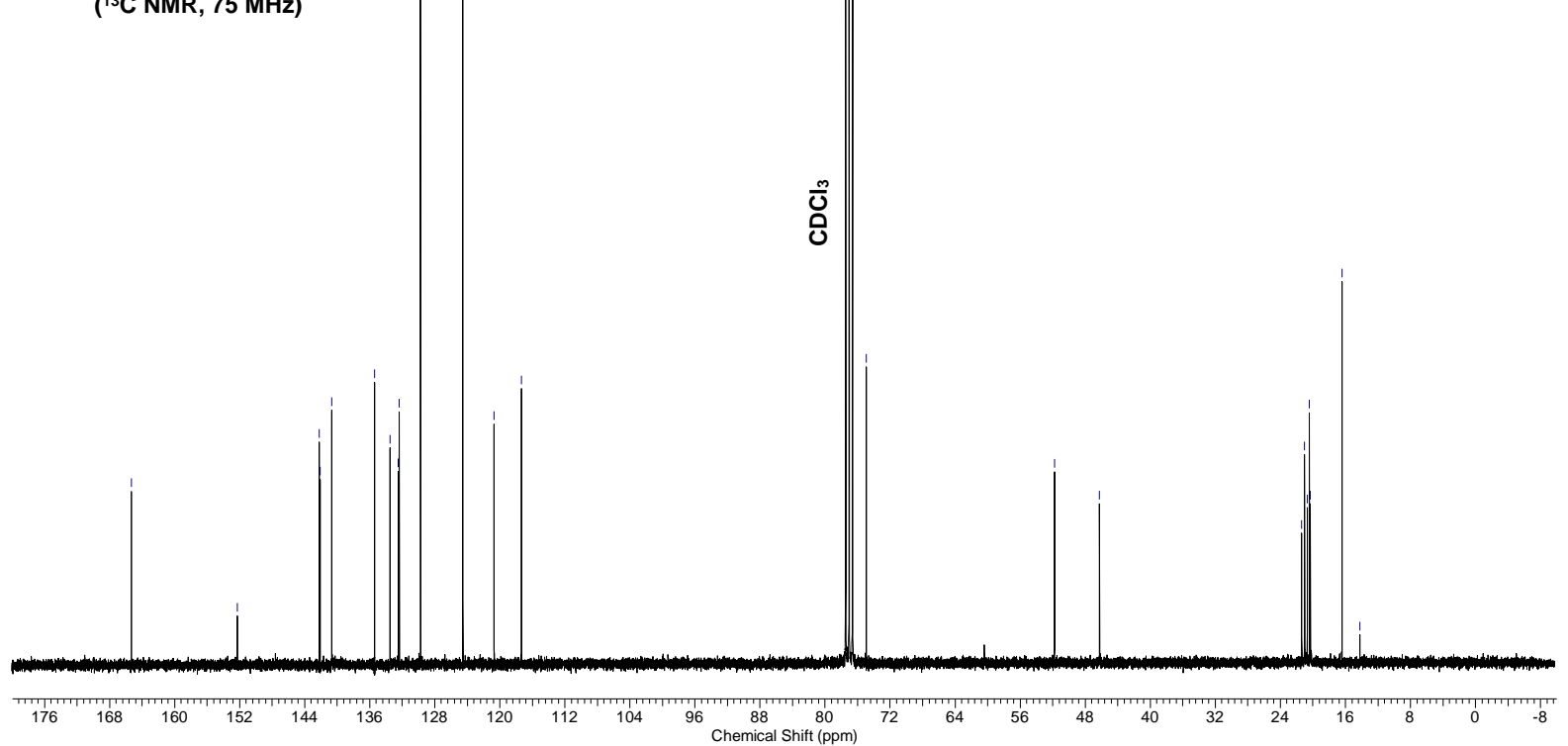




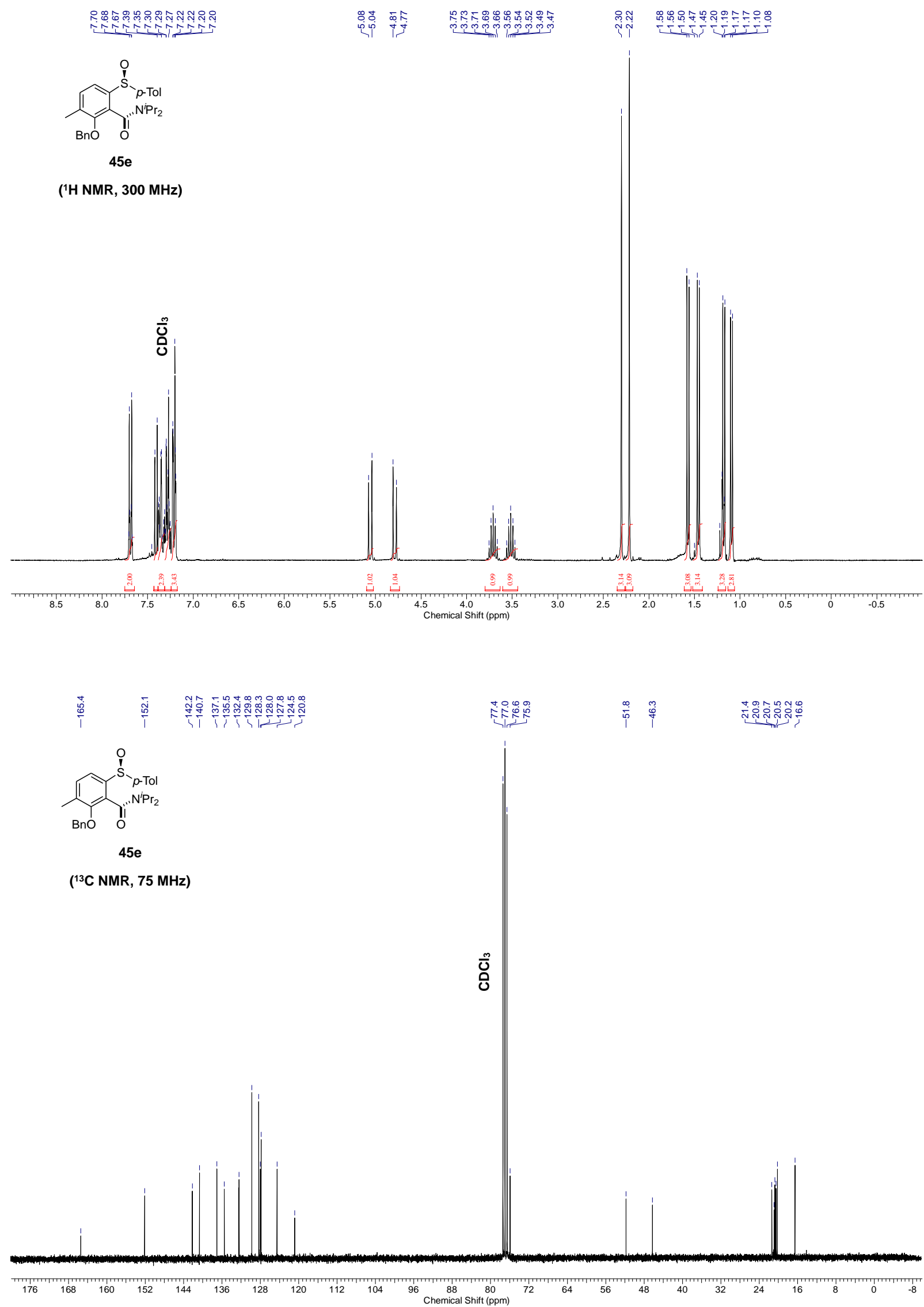




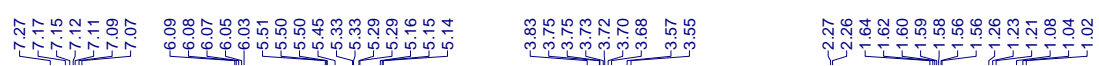<smiles>C=CC(O)c1ccc(C)c(OC)c1C(=O)[N+](=O)[O-]</smiles>

$46 f$

( $\left.{ }^{\mathrm{H}} \mathrm{NMR}, 300 \mathrm{MHz}\right)$

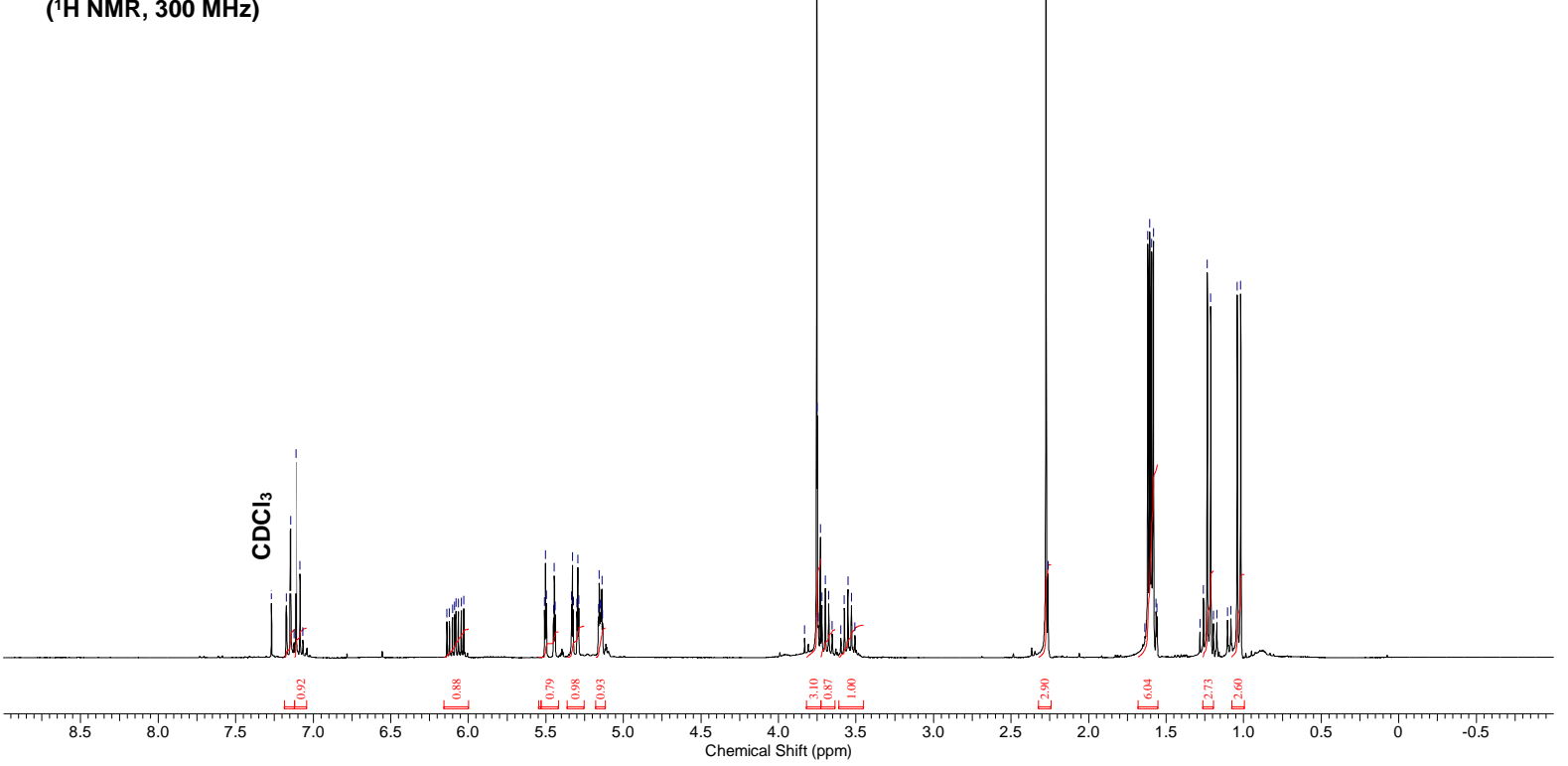

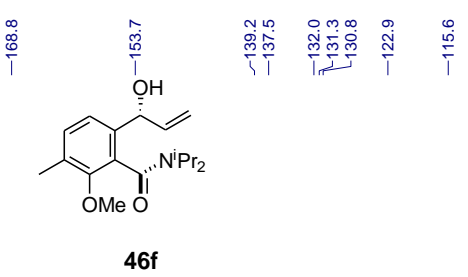

( ${ }^{13} \mathrm{C}$ NMR, $\left.75 \mathrm{MHz}\right)$

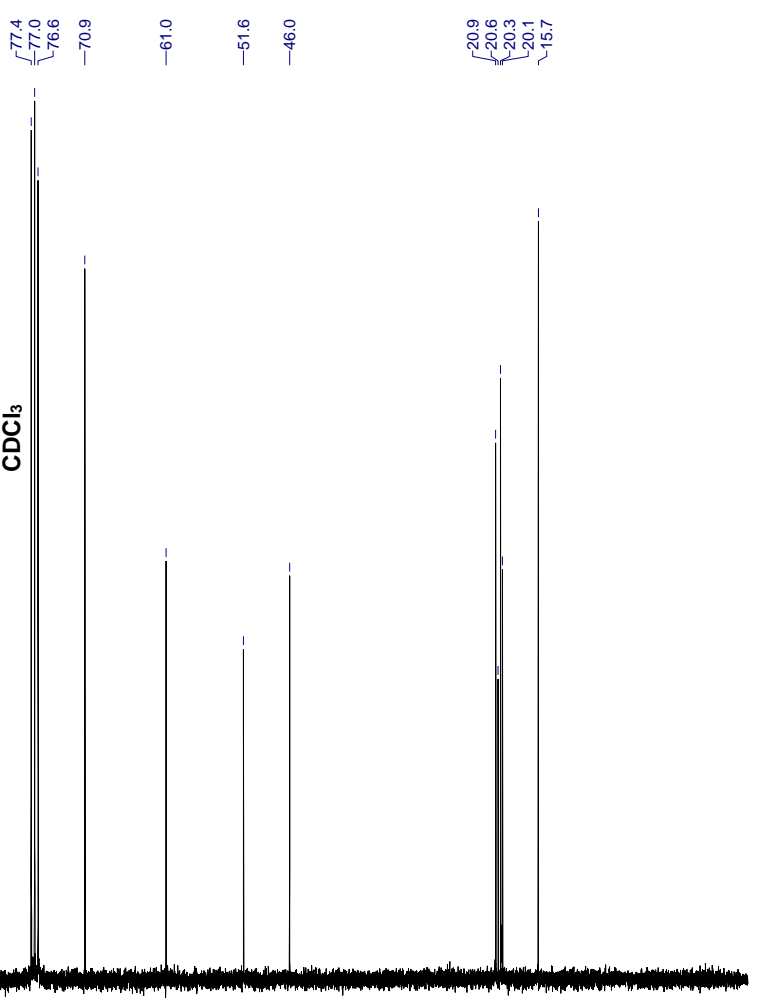

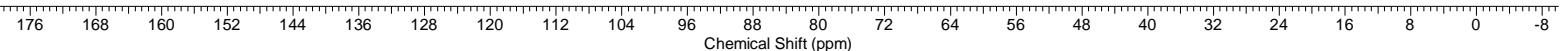




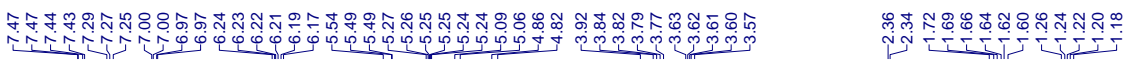

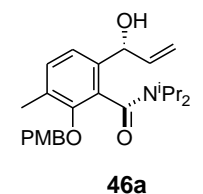

('H NMR, $300 \mathrm{MHz}$ )

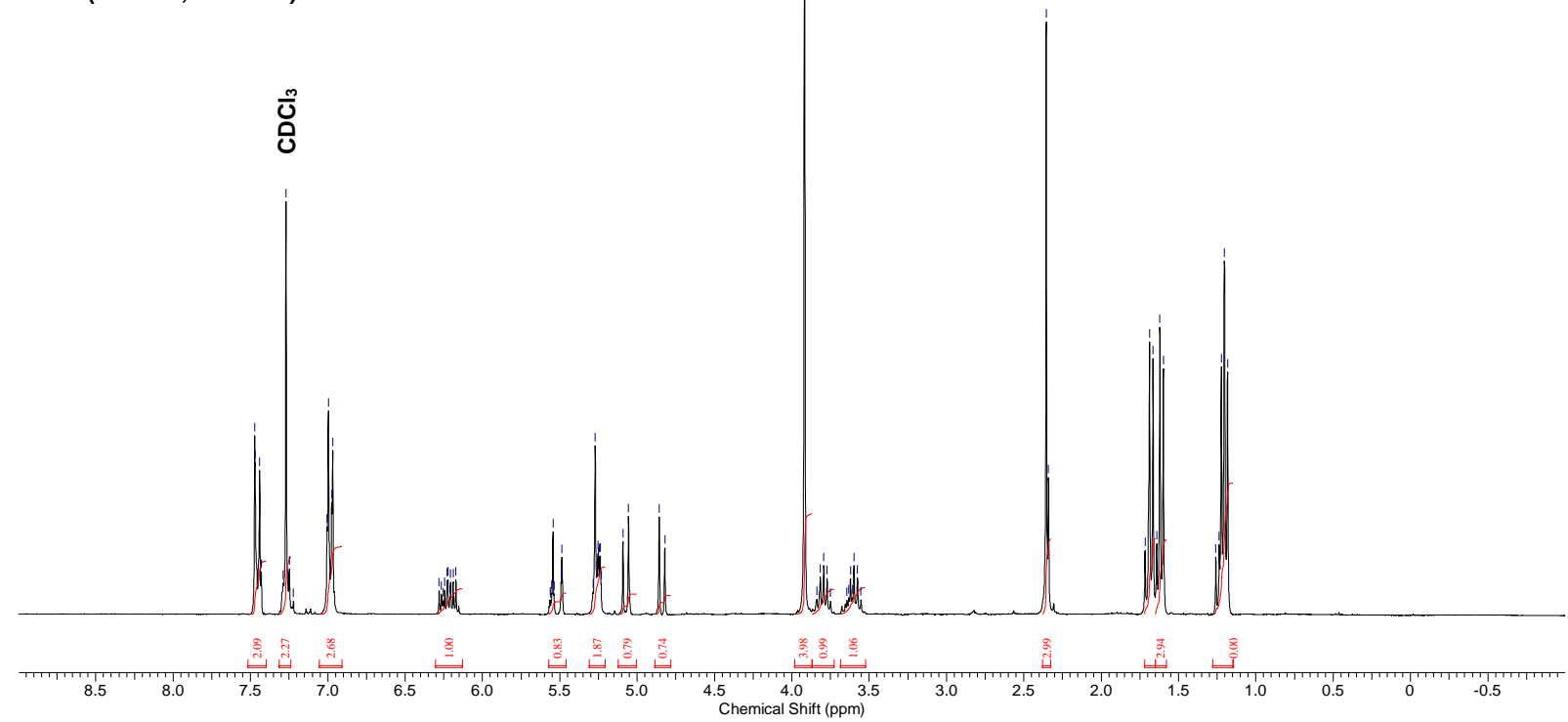

잉

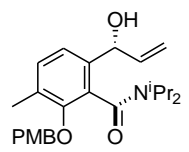

$46 a$

( ${ }^{13} \mathrm{C}$ NMR, $\left.75 \mathrm{MHz}\right)$

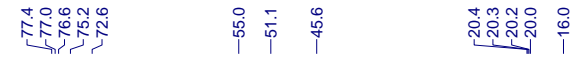

ठั

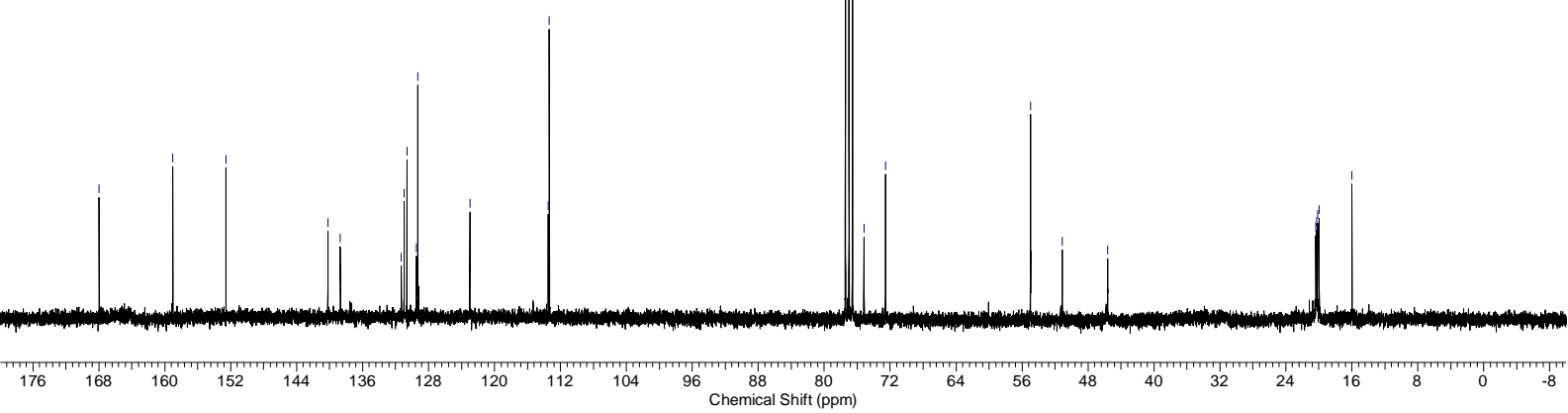




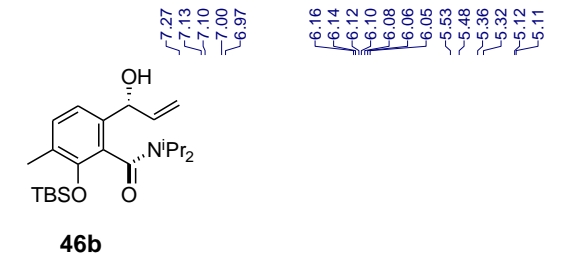

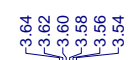

彳亍人

( ${ }^{1} \mathrm{H}$ NMR, 300 MHz)
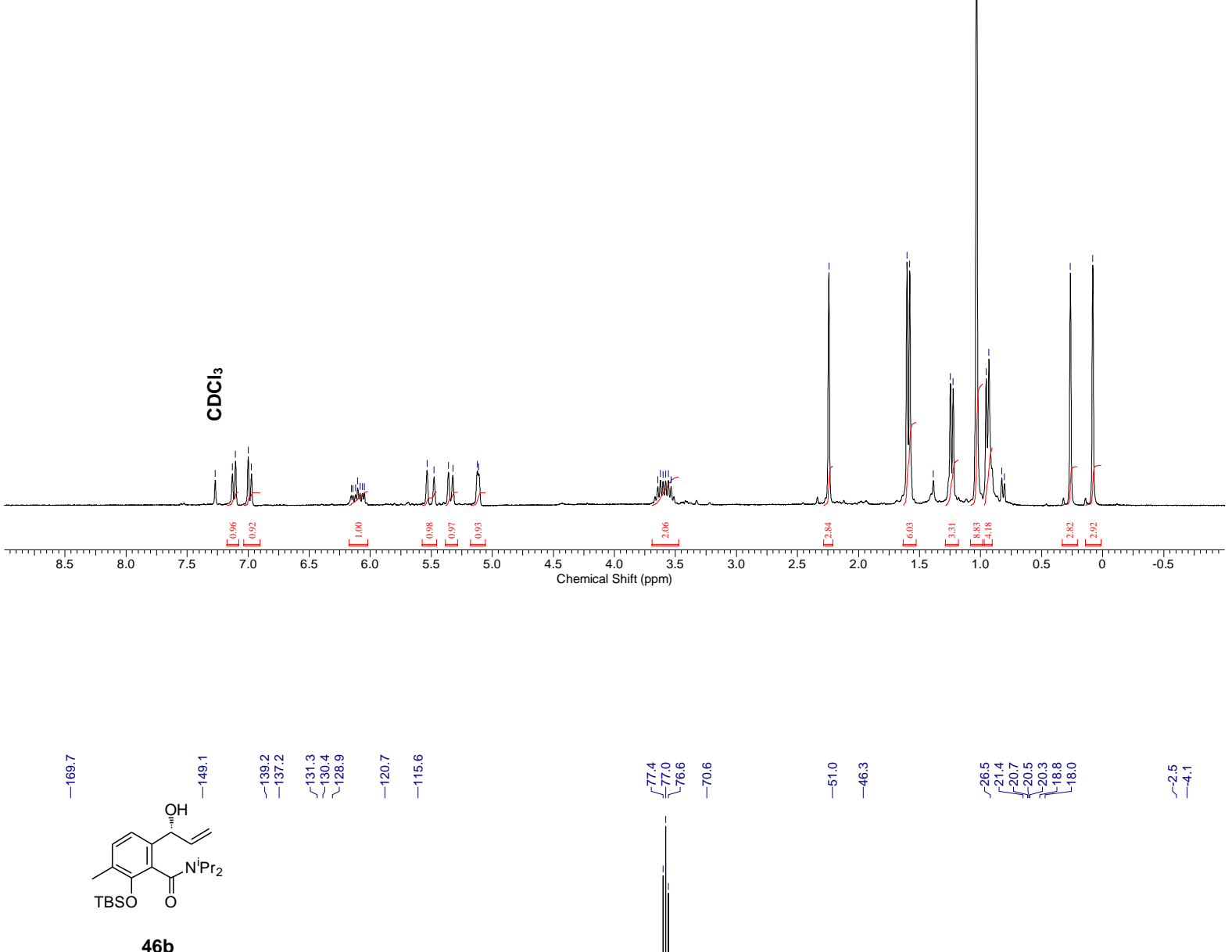

( ${ }^{13} \mathrm{C}$ NMR, $75 \mathrm{MHz}$ )

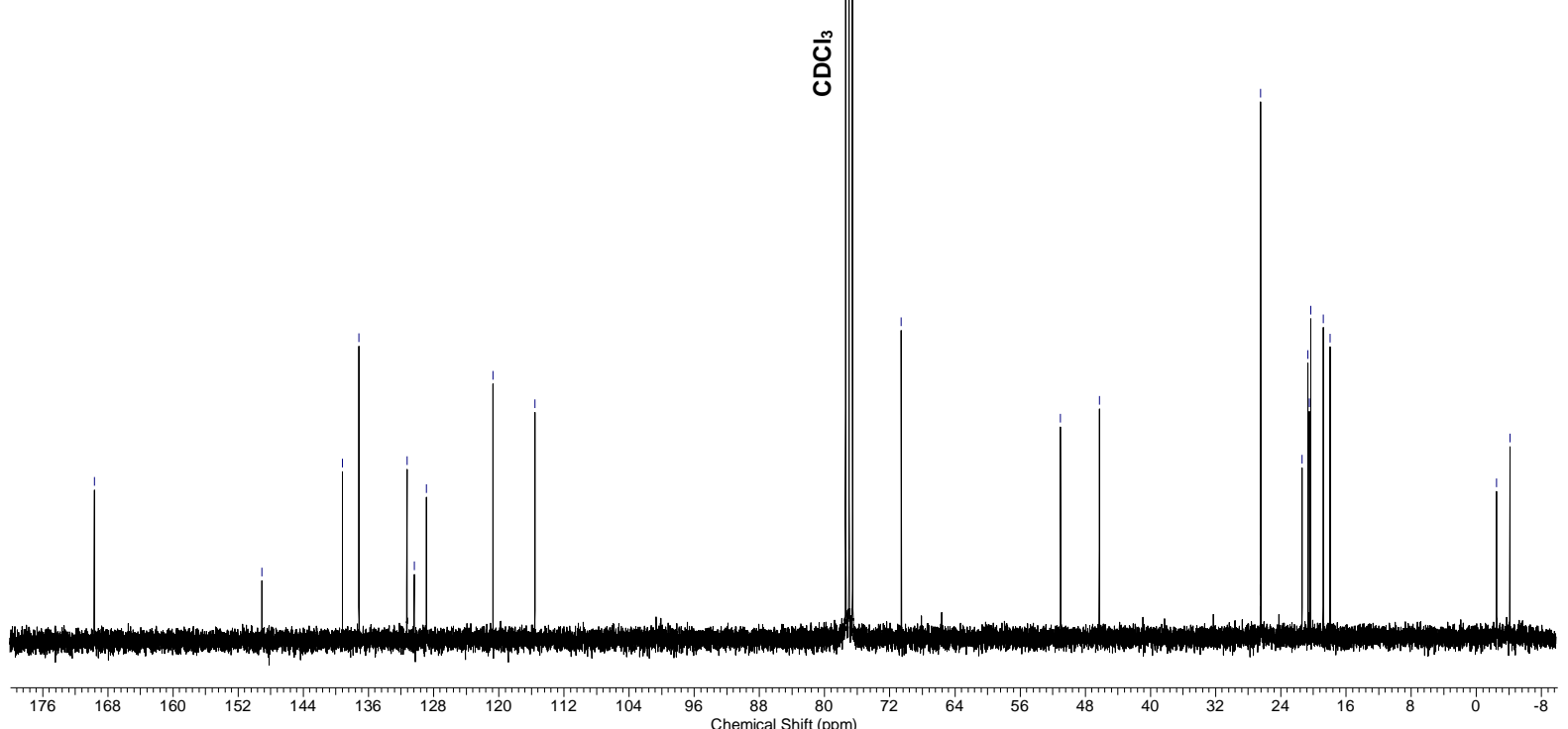




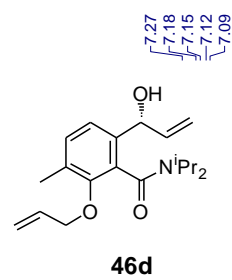

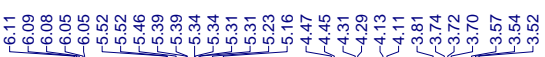

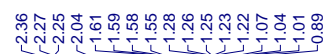

('H NMR, 300 MHz)

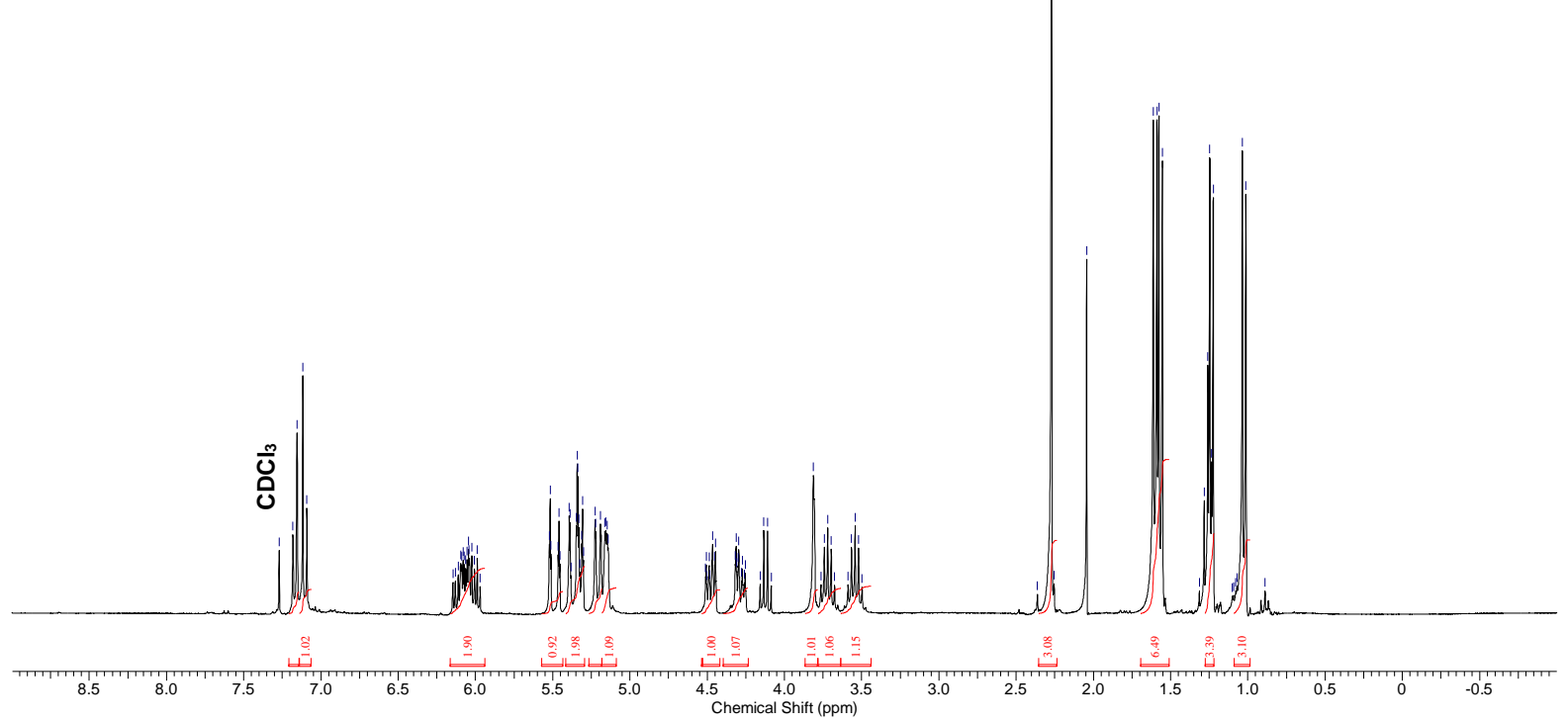

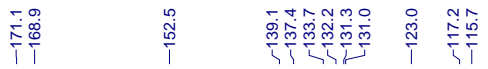

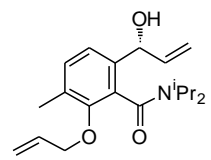

46d

$\left({ }^{13} \mathrm{C} \mathrm{NMR}, 75 \mathrm{MHz}\right)$

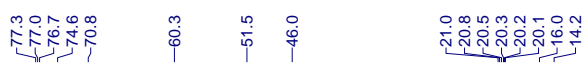

ठํํㅇ 


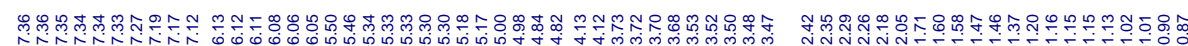

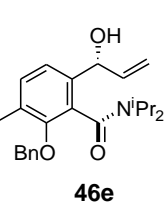

('H NMR, 400 MHz)

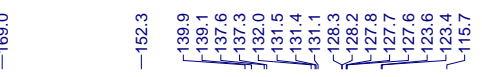

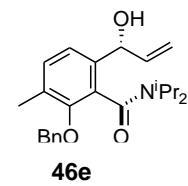

( ${ }^{13} \mathrm{C}$ NMR, $\left.101 \mathrm{MHz}\right)$

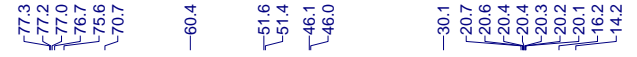

రั๊
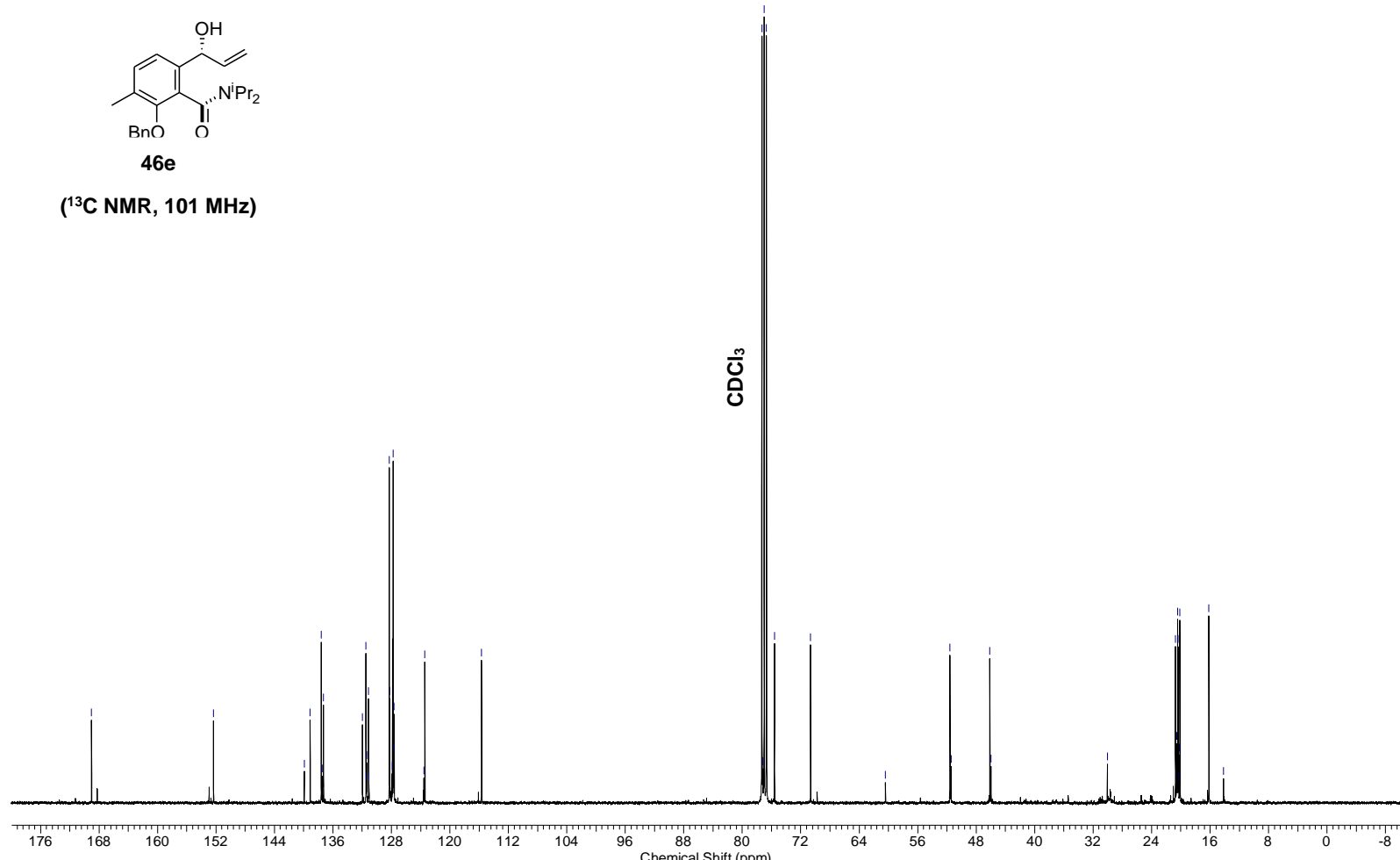


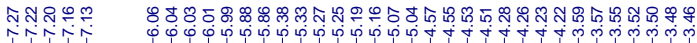

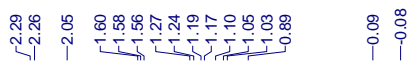

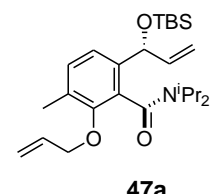

('H NMR, $300 \mathrm{MHz}$ )

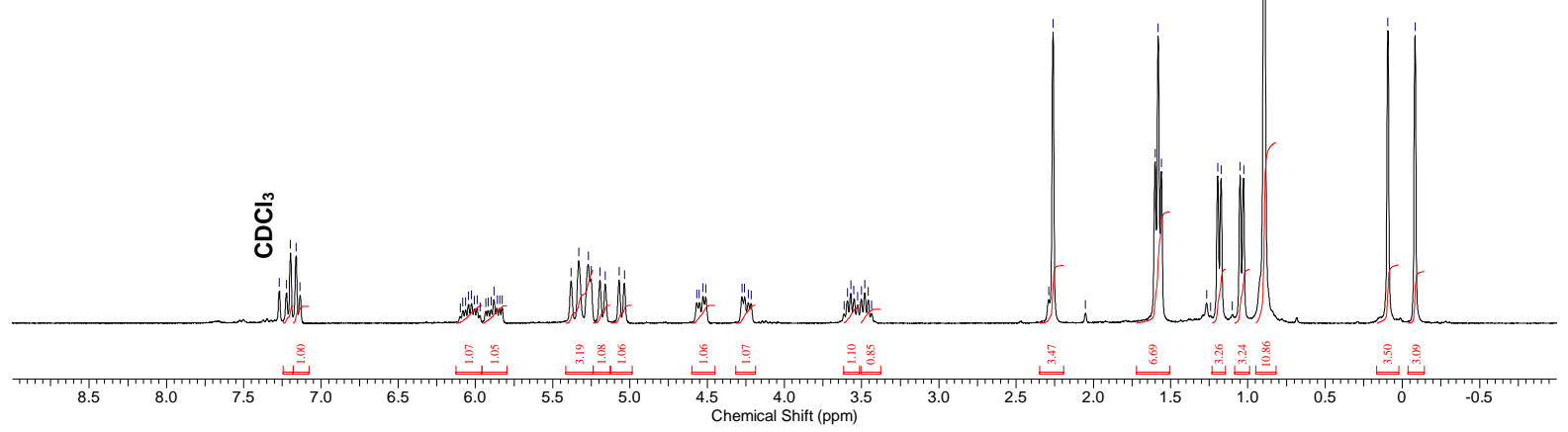

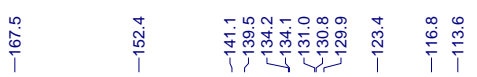

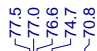

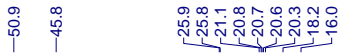

$\stackrel{\infty}{i=0}$

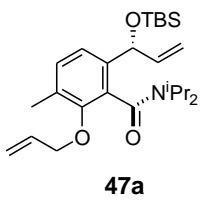

( ${ }^{13} \mathrm{C}$ NMR, $\left.75 \mathrm{MHz}\right)$

రั0

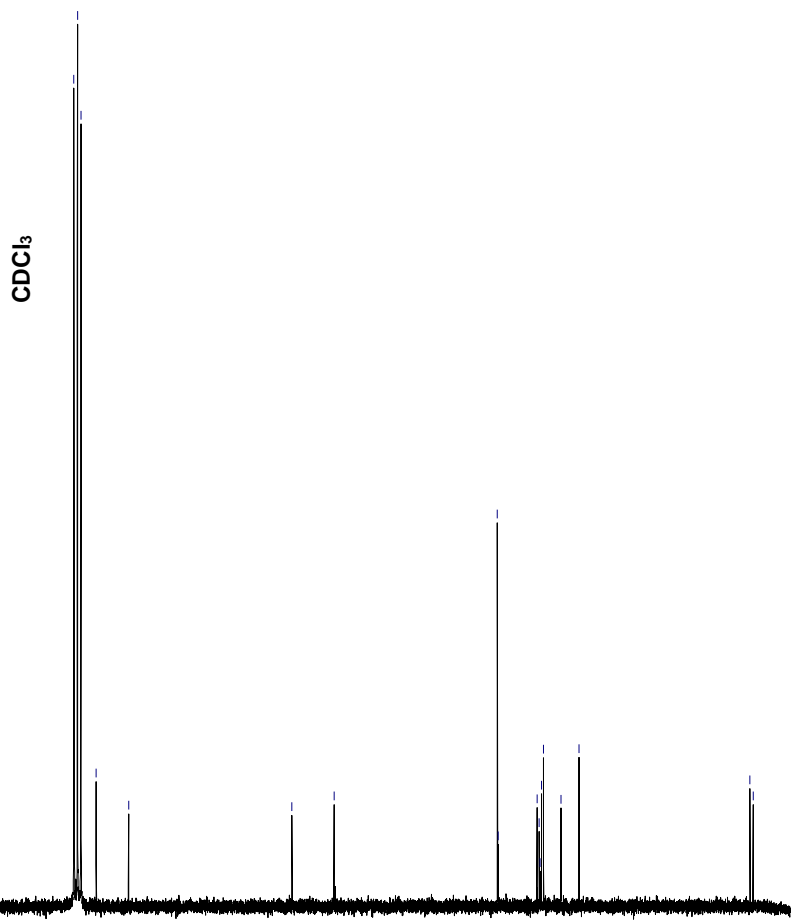

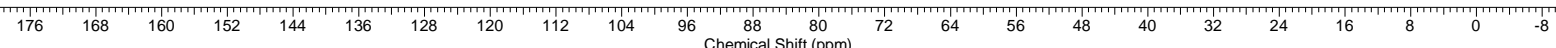



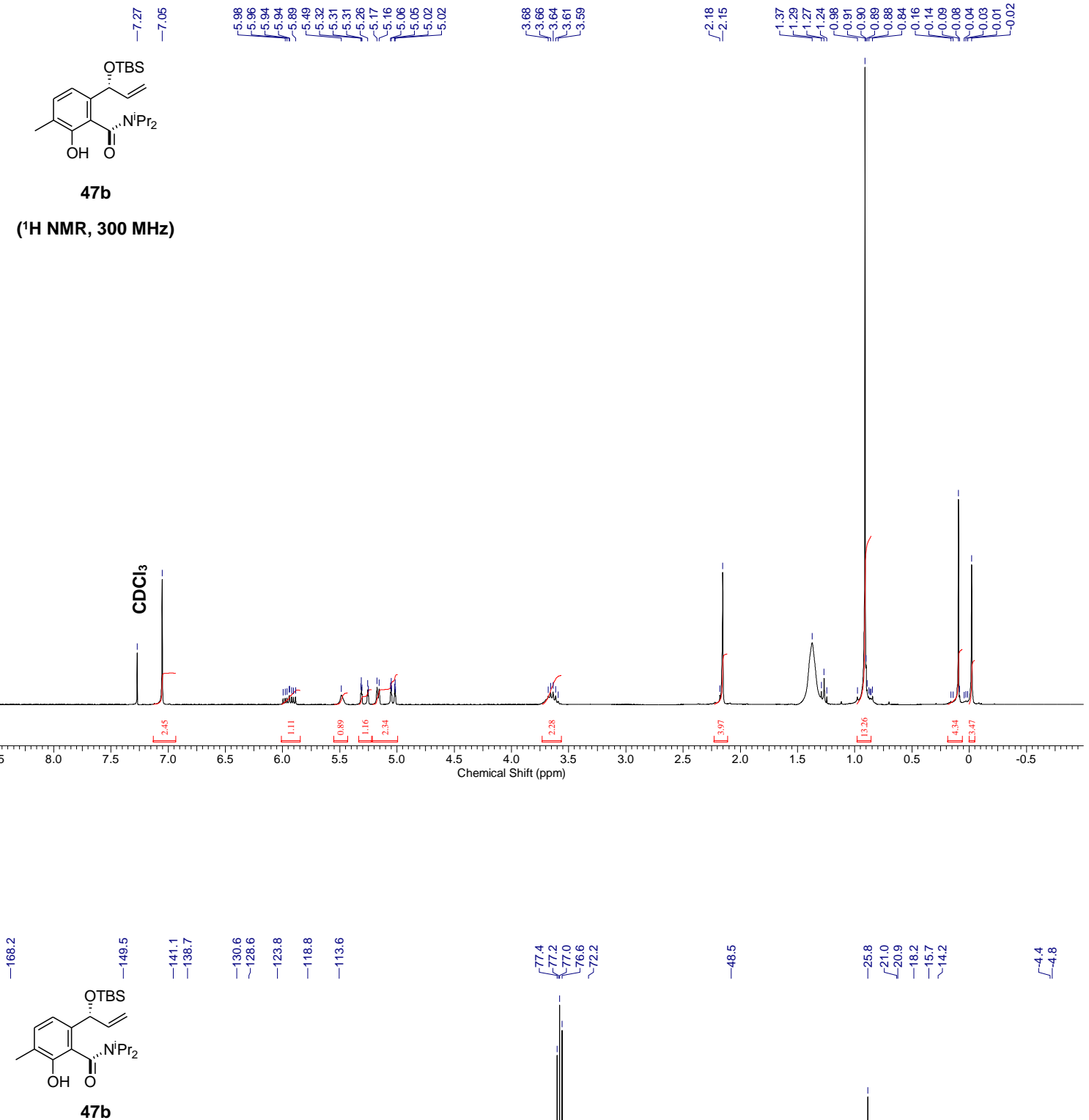

( ${ }^{13} \mathrm{C}$ NMR, $75 \mathrm{MHz}$ )

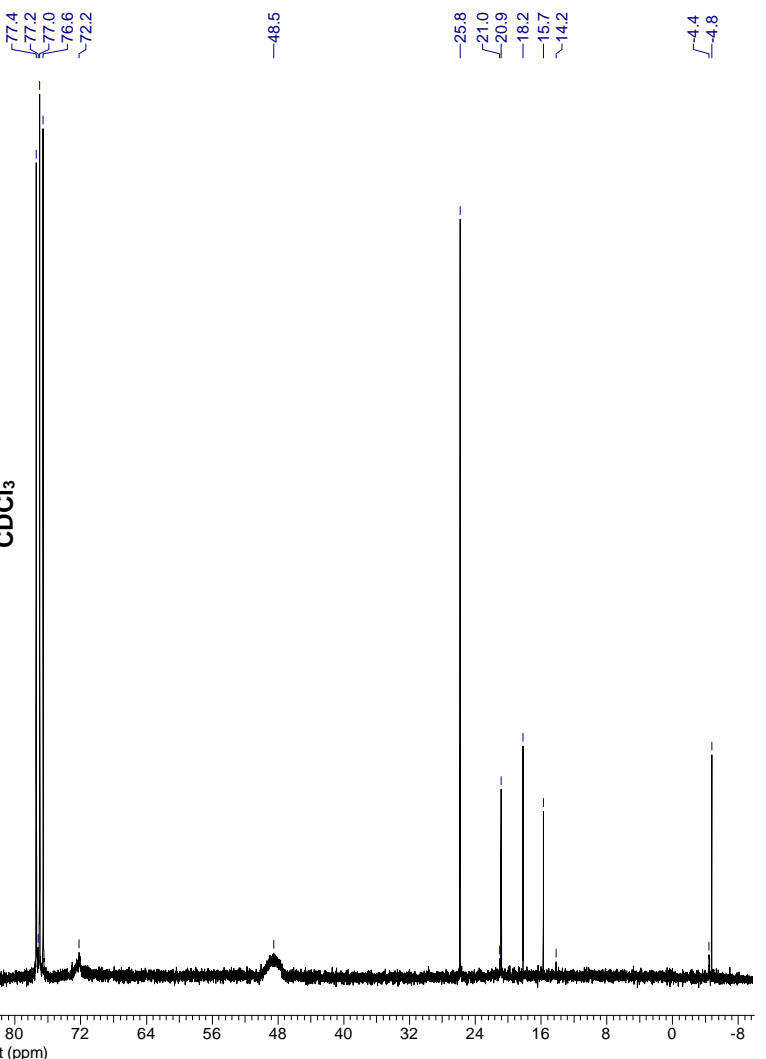



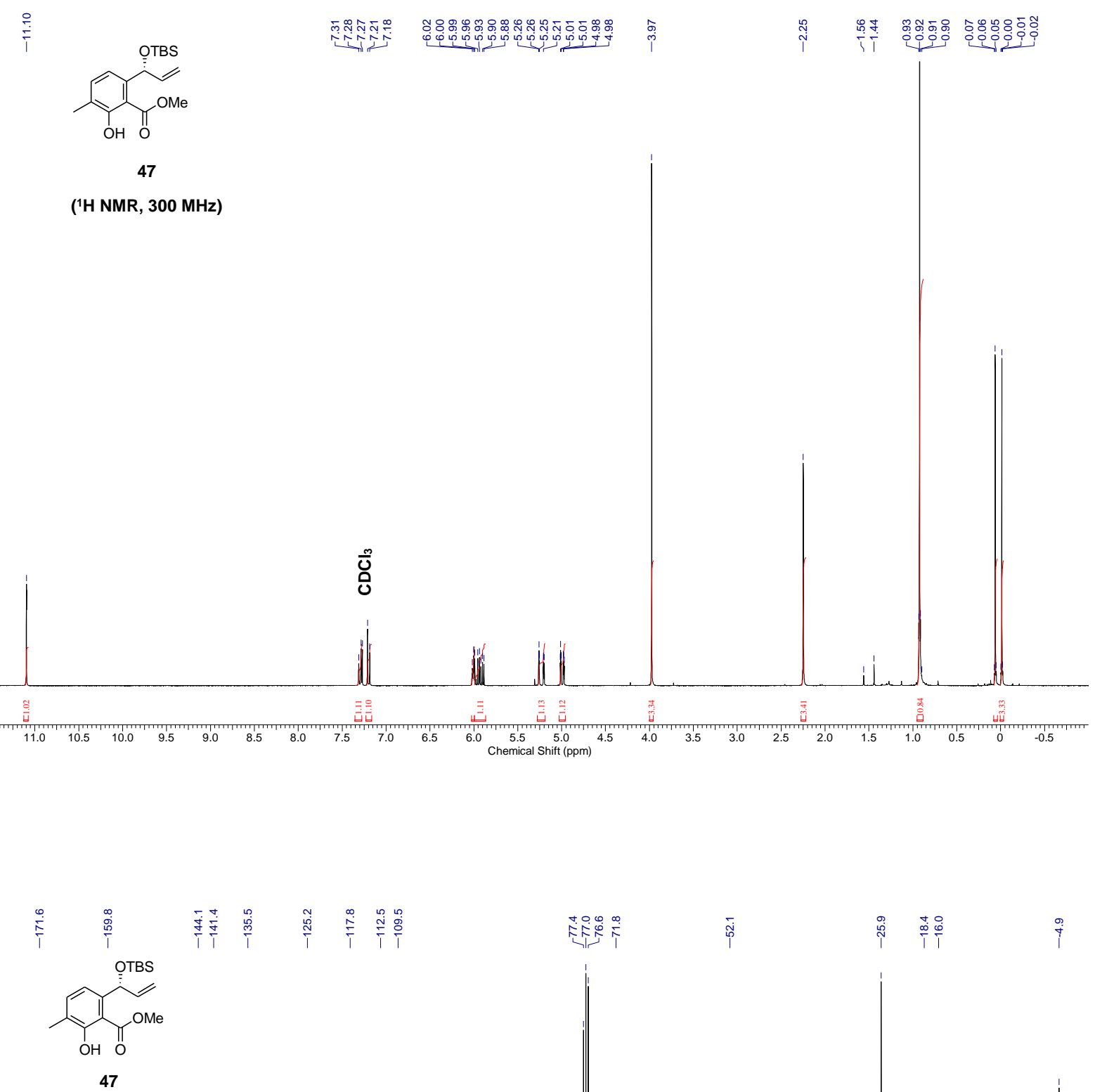

$\left({ }^{13} \mathrm{C}\right.$ NMR, $\left.75 \mathrm{MHz}\right)$

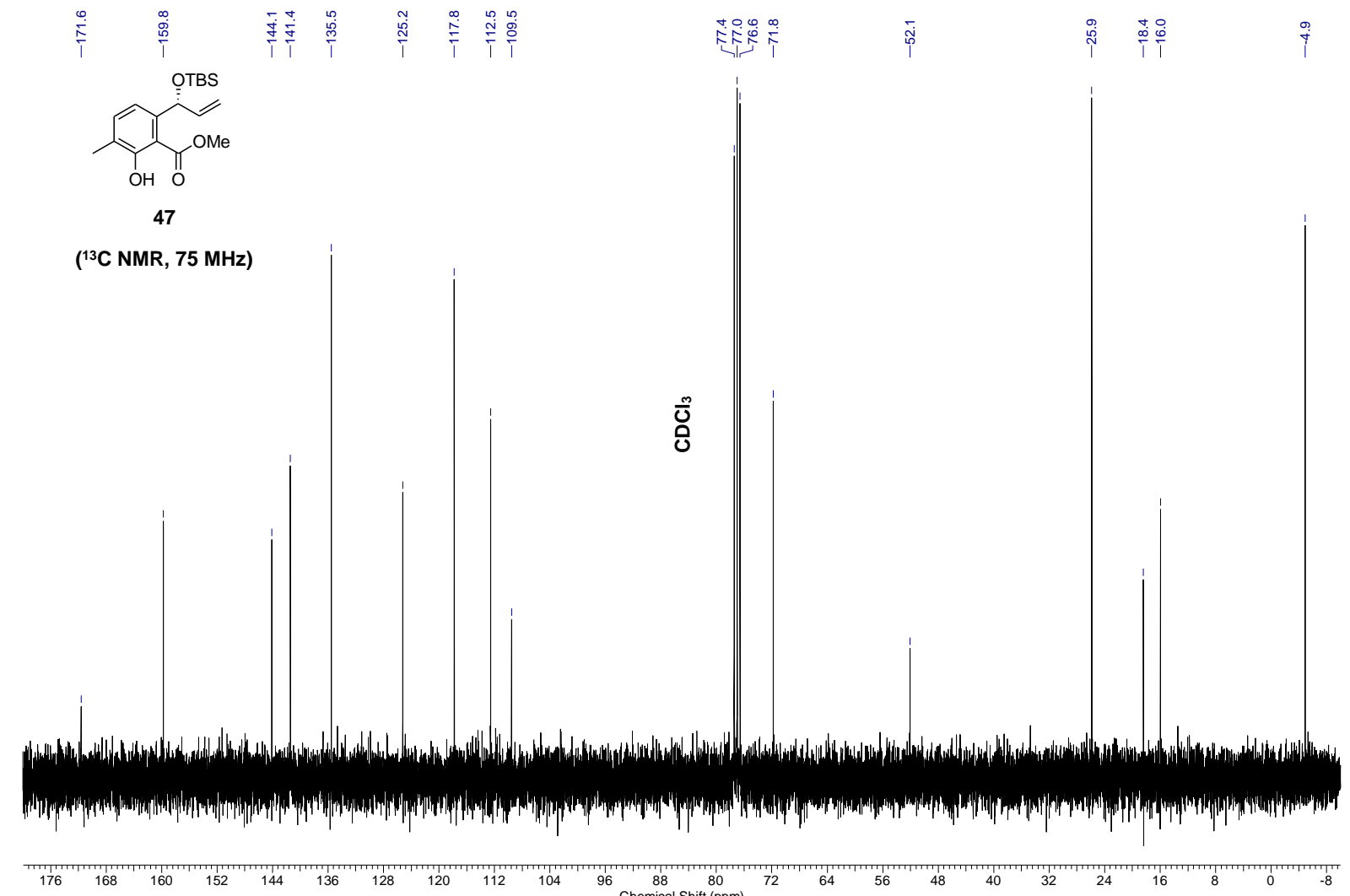

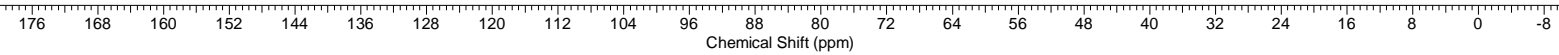




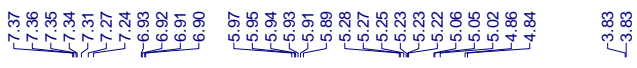
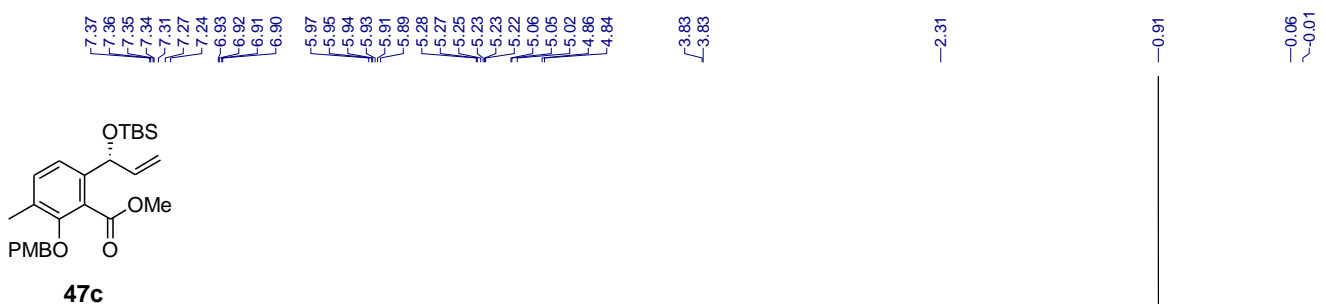

('H NMR, $300 \mathrm{MHz}$ )

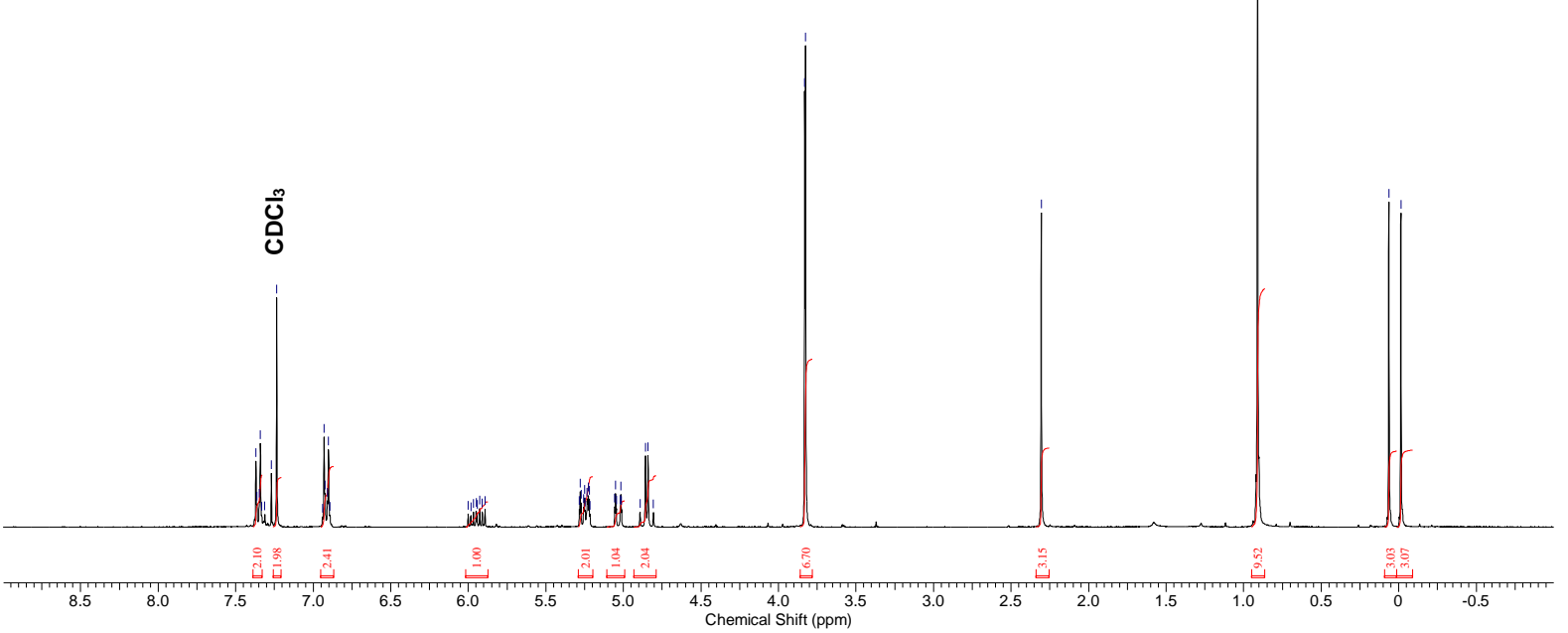

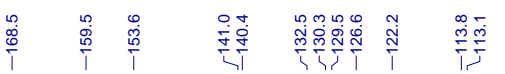

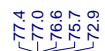

帤菖

苗

$\stackrel{\circ}{\dot{\varphi}}$

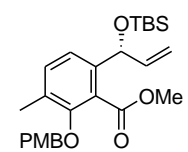

47c

(133 NMR, $75 \mathrm{MHz}$ )

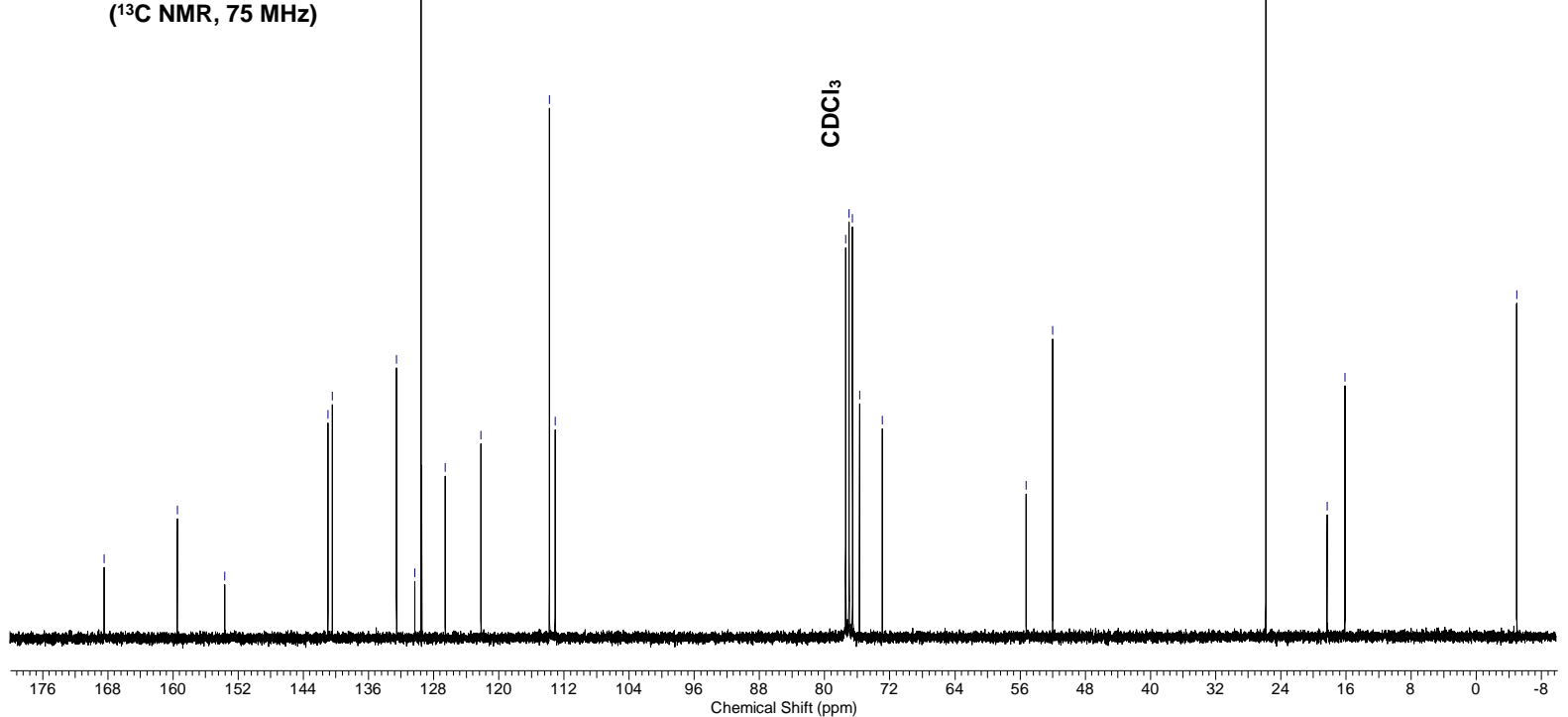


:

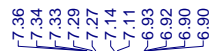

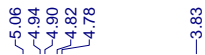

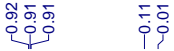

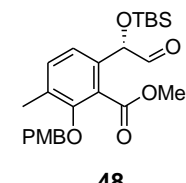

('H NMR, 300 MHz)

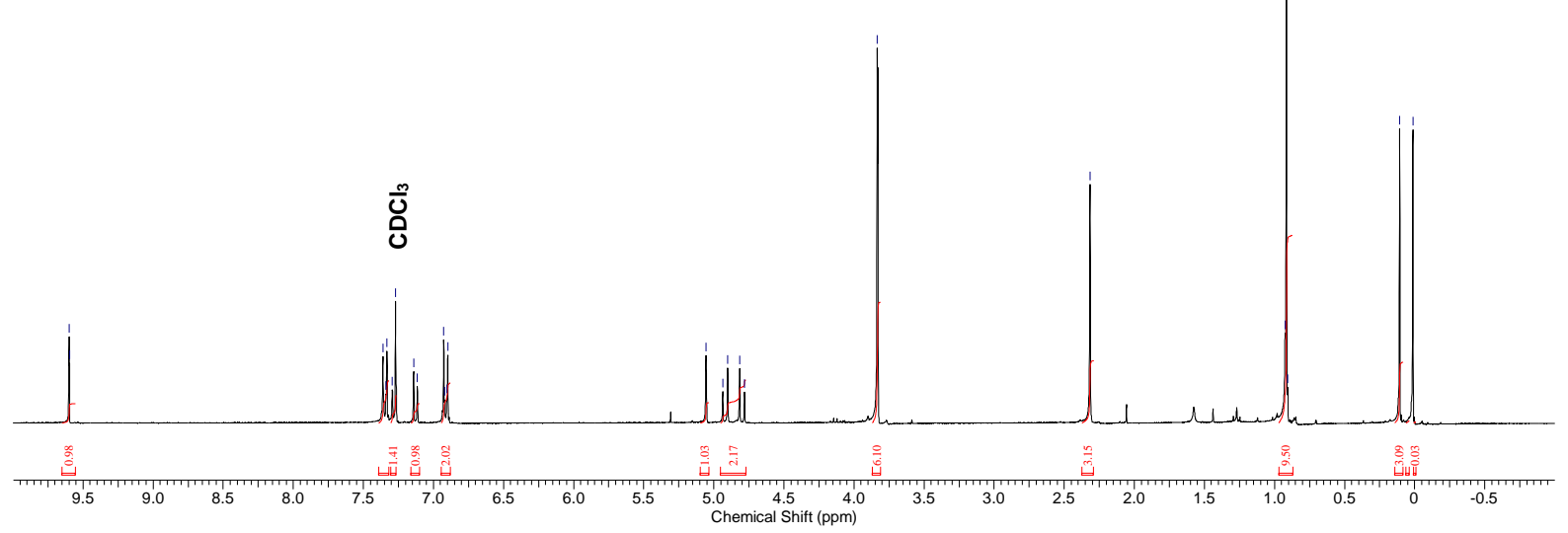

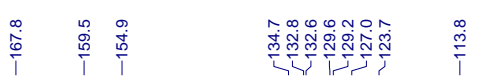

ํํำ

ஸें

$\stackrel{0}{i}$

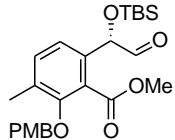

48

( ${ }^{13} \mathrm{C}$ NMR, $75 \mathrm{MHz}$ )

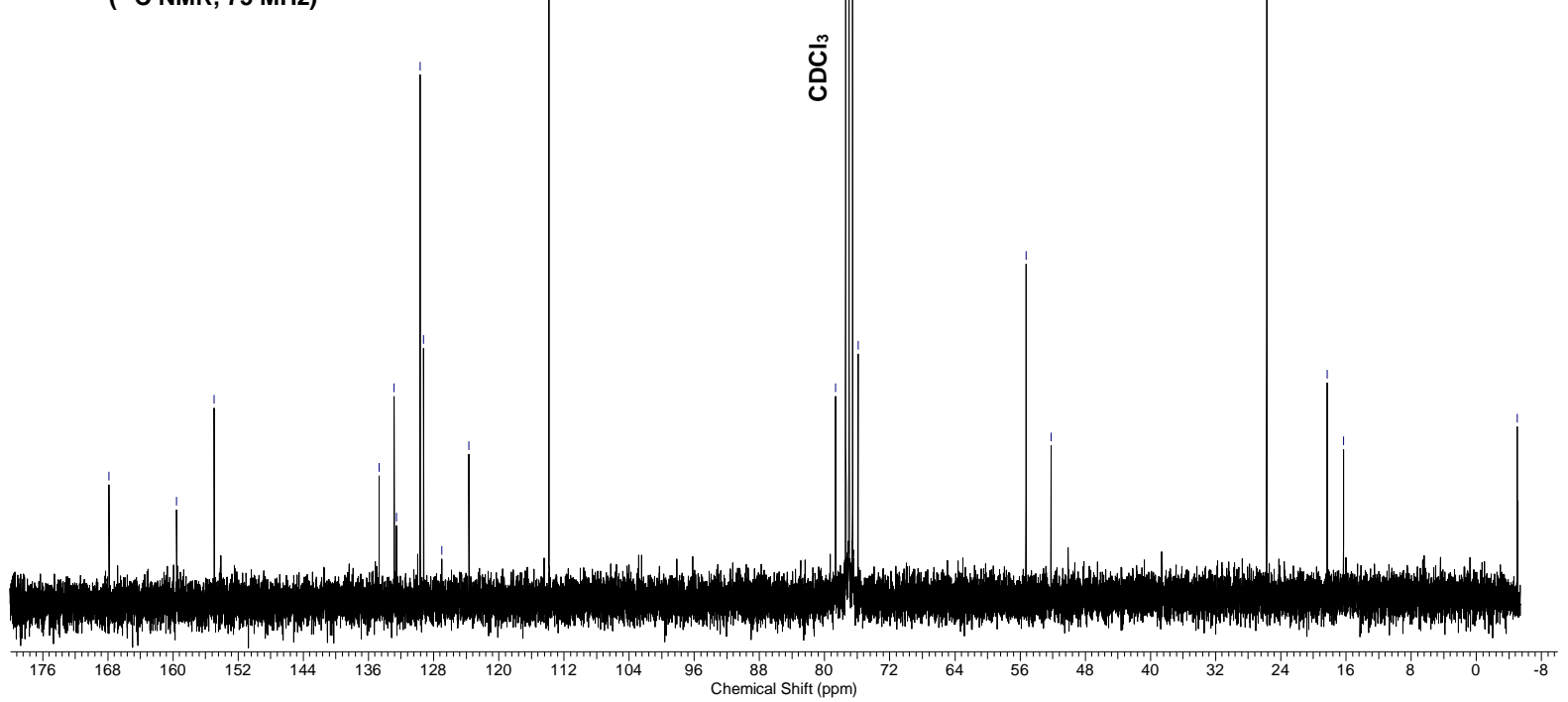



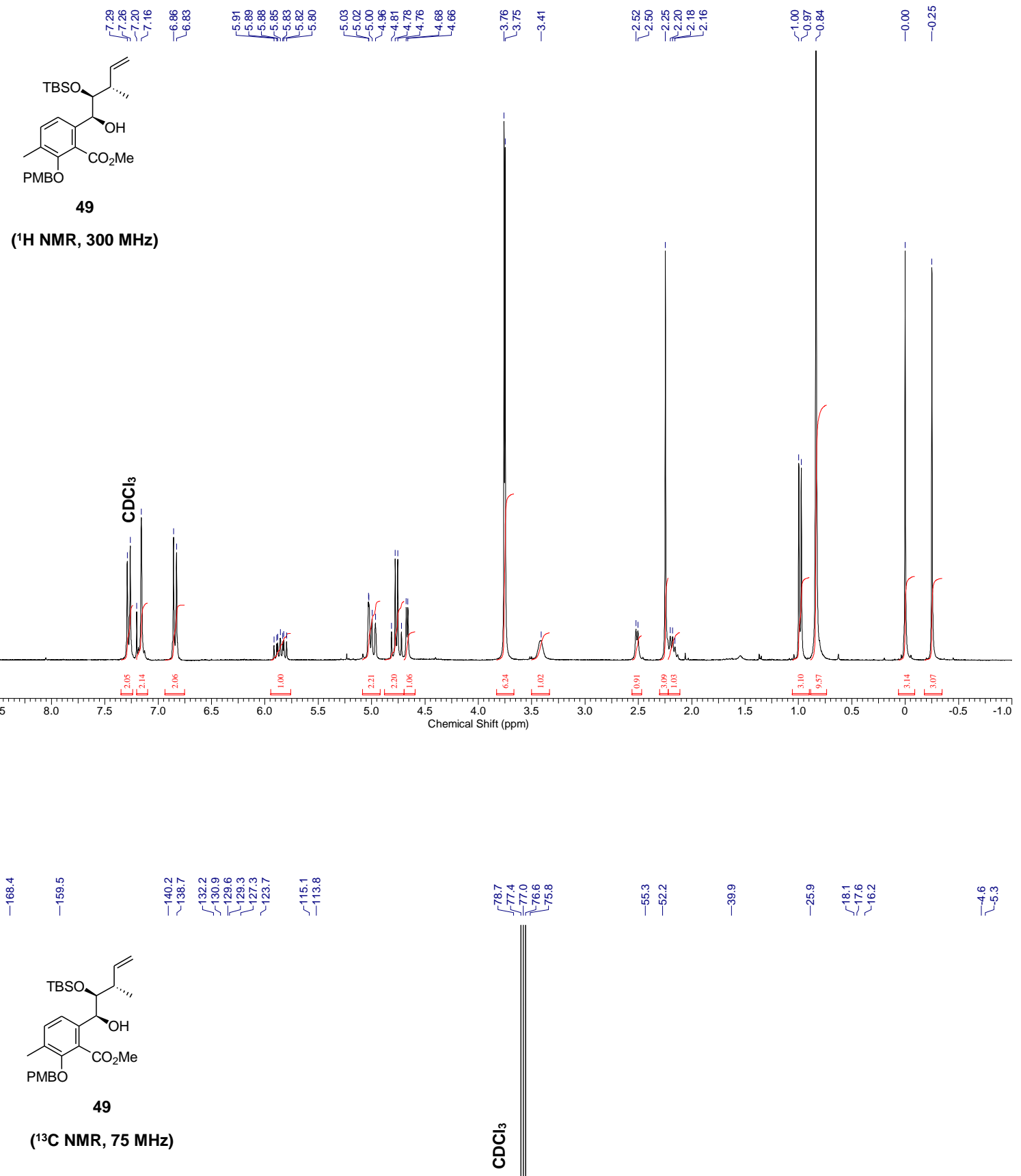

$\stackrel{0}{i} \dot{j}$

( ${ }^{13} \mathrm{C}$ NMR, $\left.75 \mathrm{MHz}\right)$ 


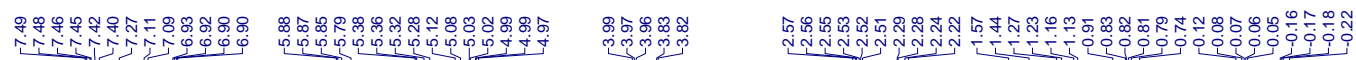

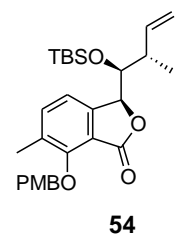

( $\left.{ }^{\mathrm{H}} \mathrm{NMR}, 300 \mathrm{MHz}\right)$

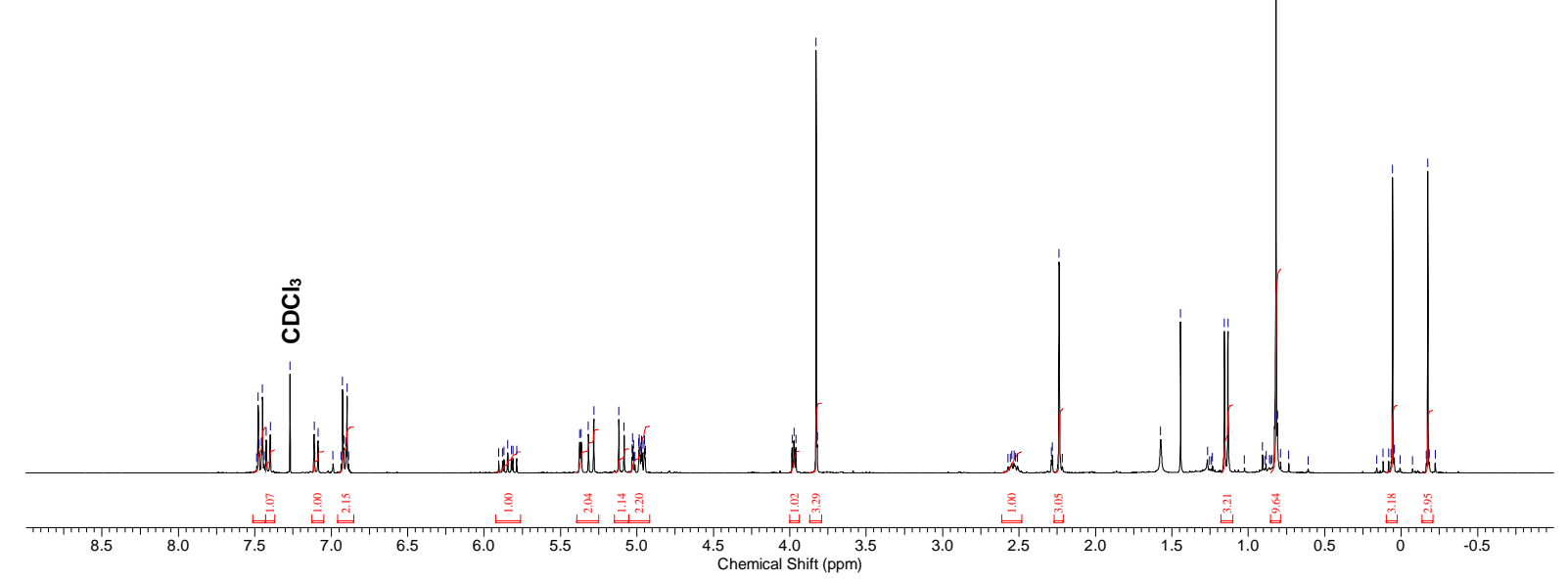

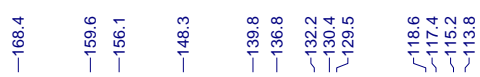
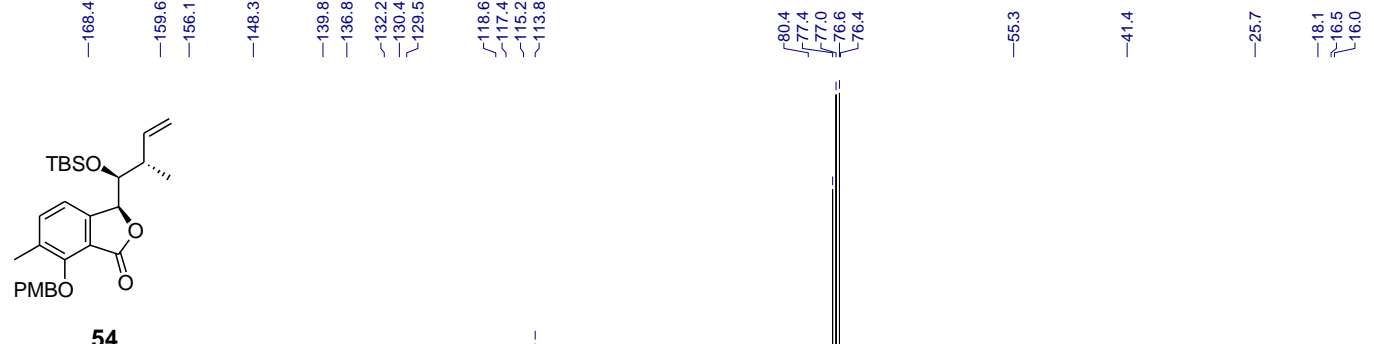

( ${ }^{13} \mathrm{C}$ NMR, $\left.75 \mathrm{MHz}\right)$

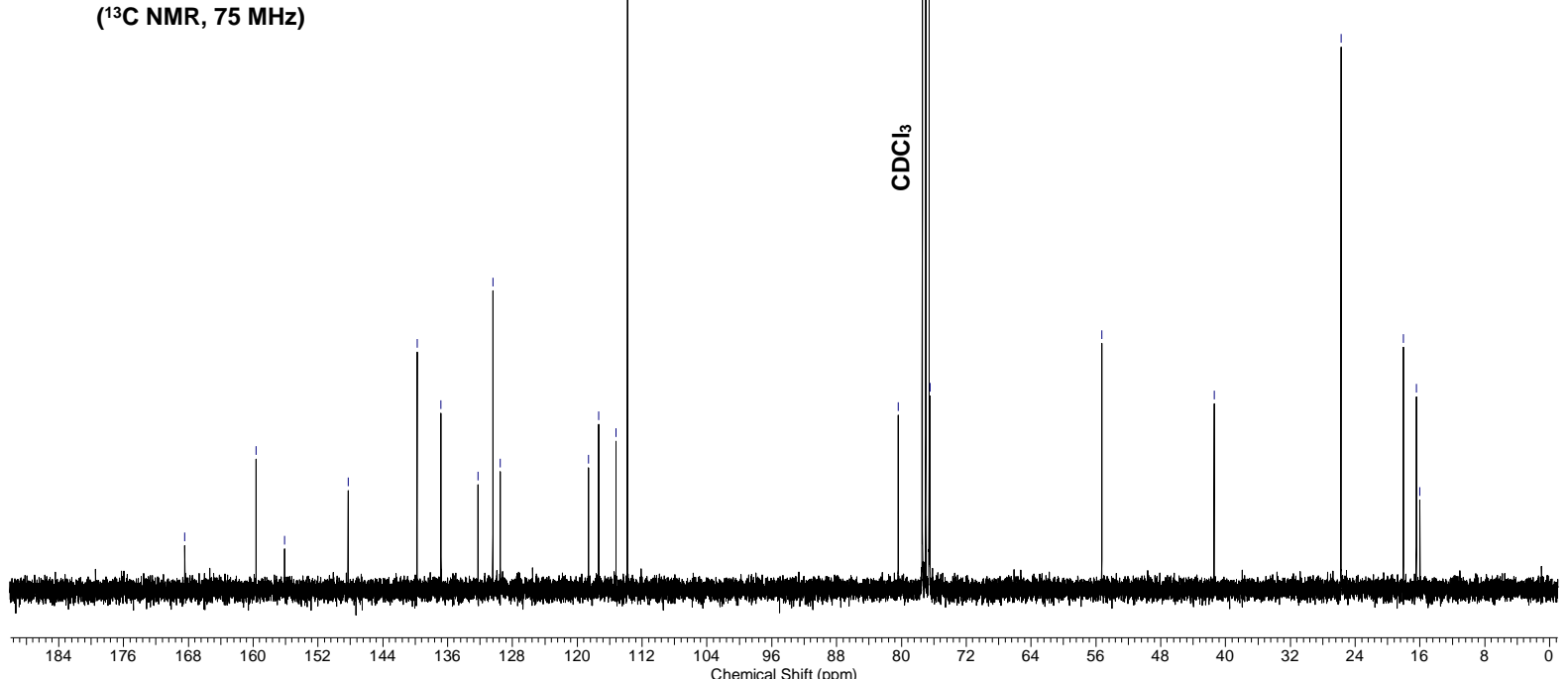




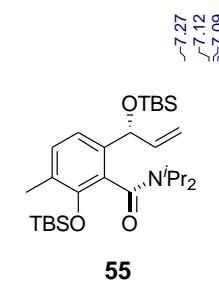

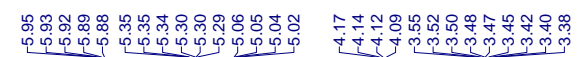

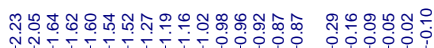

('H NMR, $300 \mathrm{MHz}$ )

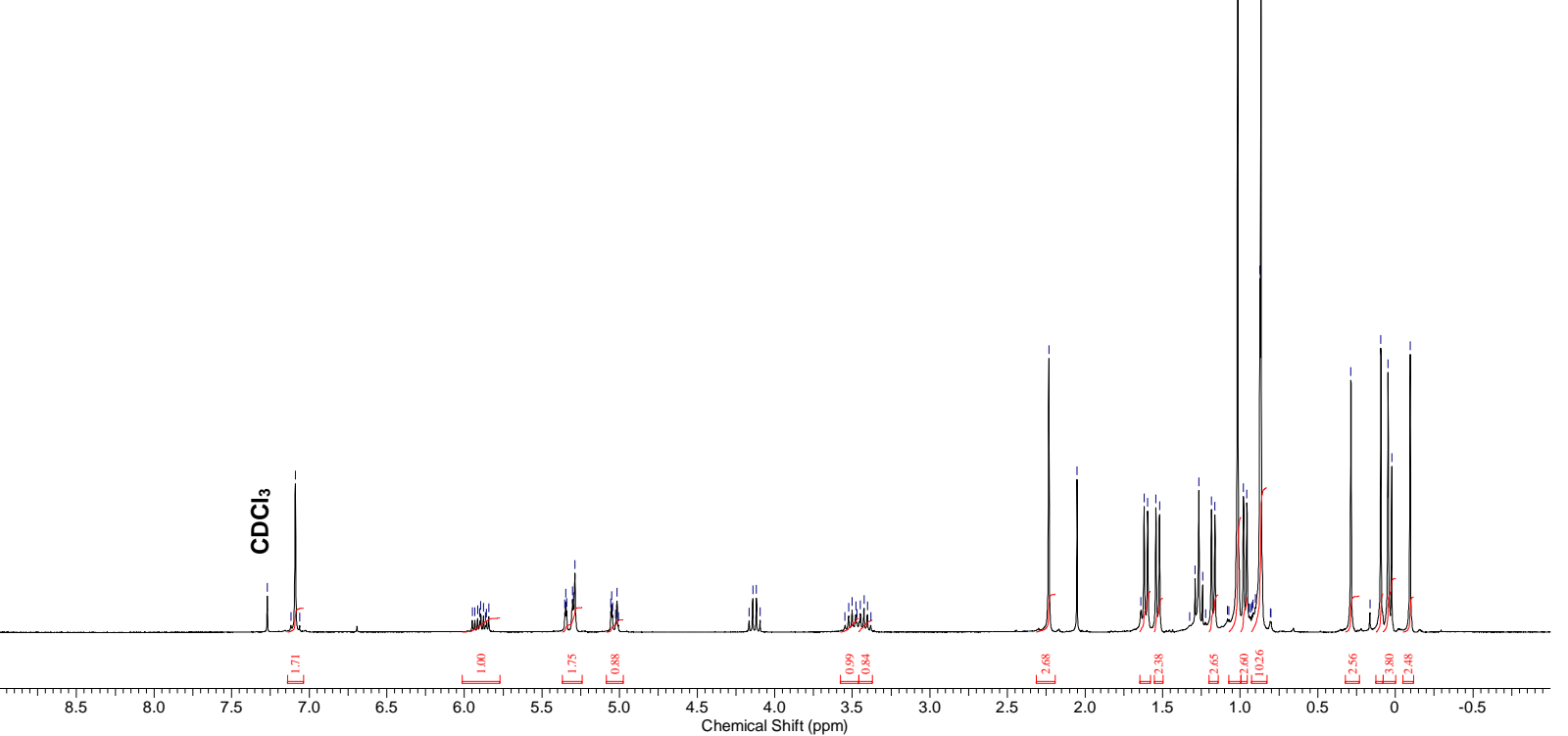

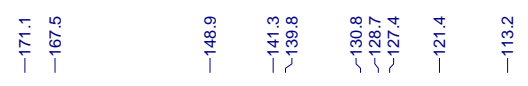

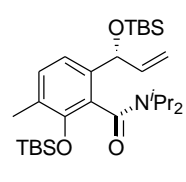

55

( ${ }^{13} \mathrm{C}$ NMR, $\left.75 \mathrm{MHz}\right)$

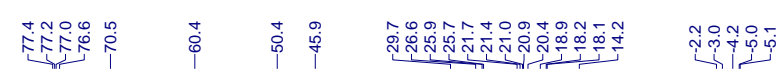

这

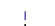




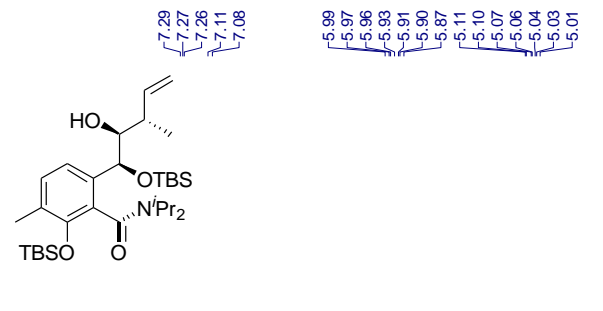

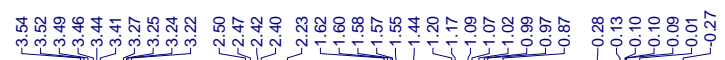

57

('H NMR, $300 \mathrm{MHz}$ )

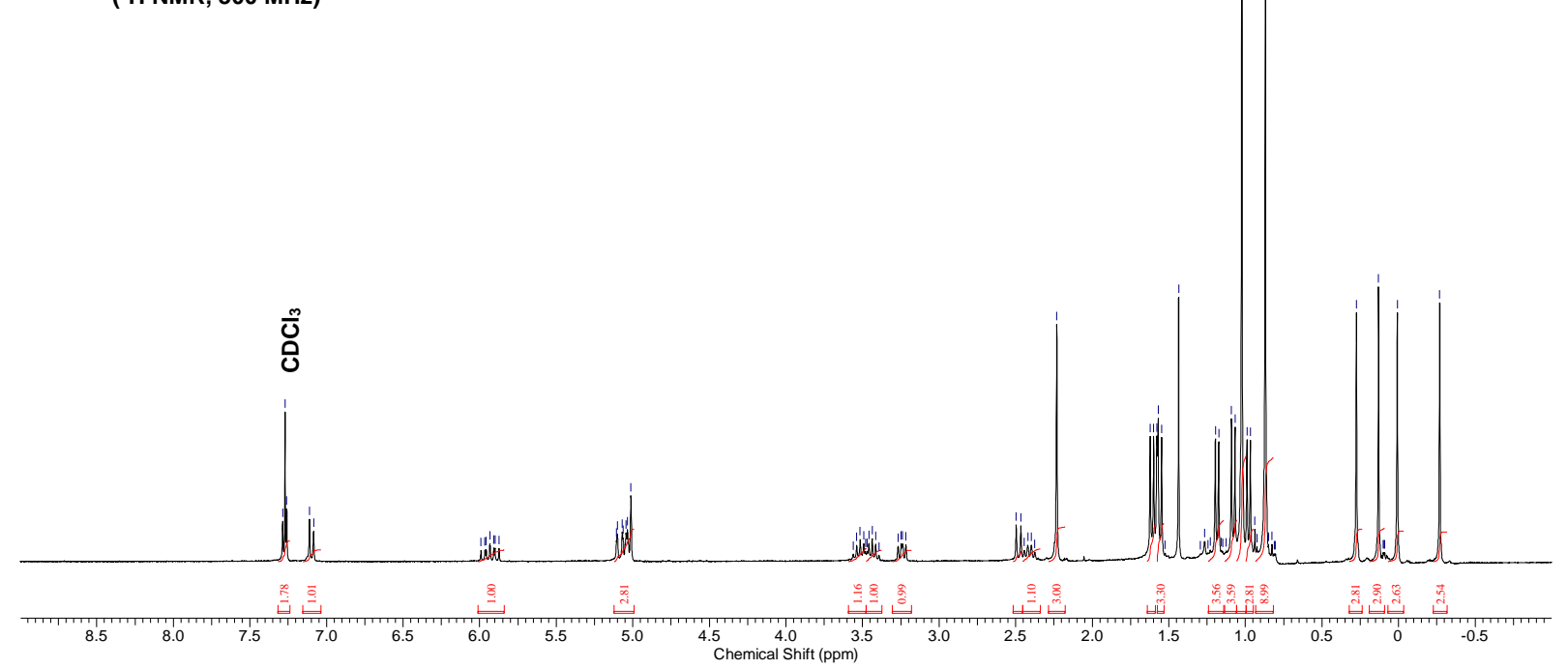

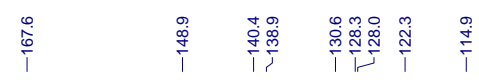

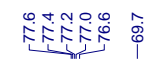

告

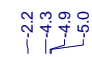

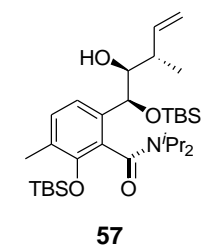

( ${ }^{13} \mathrm{C}$ NMR, $\left.75 \mathrm{MHz}\right)$

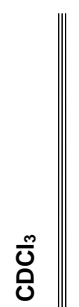

రั

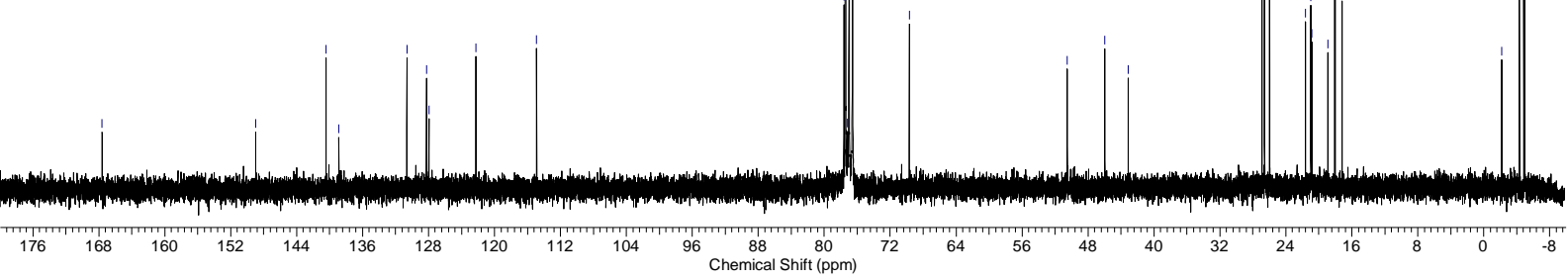



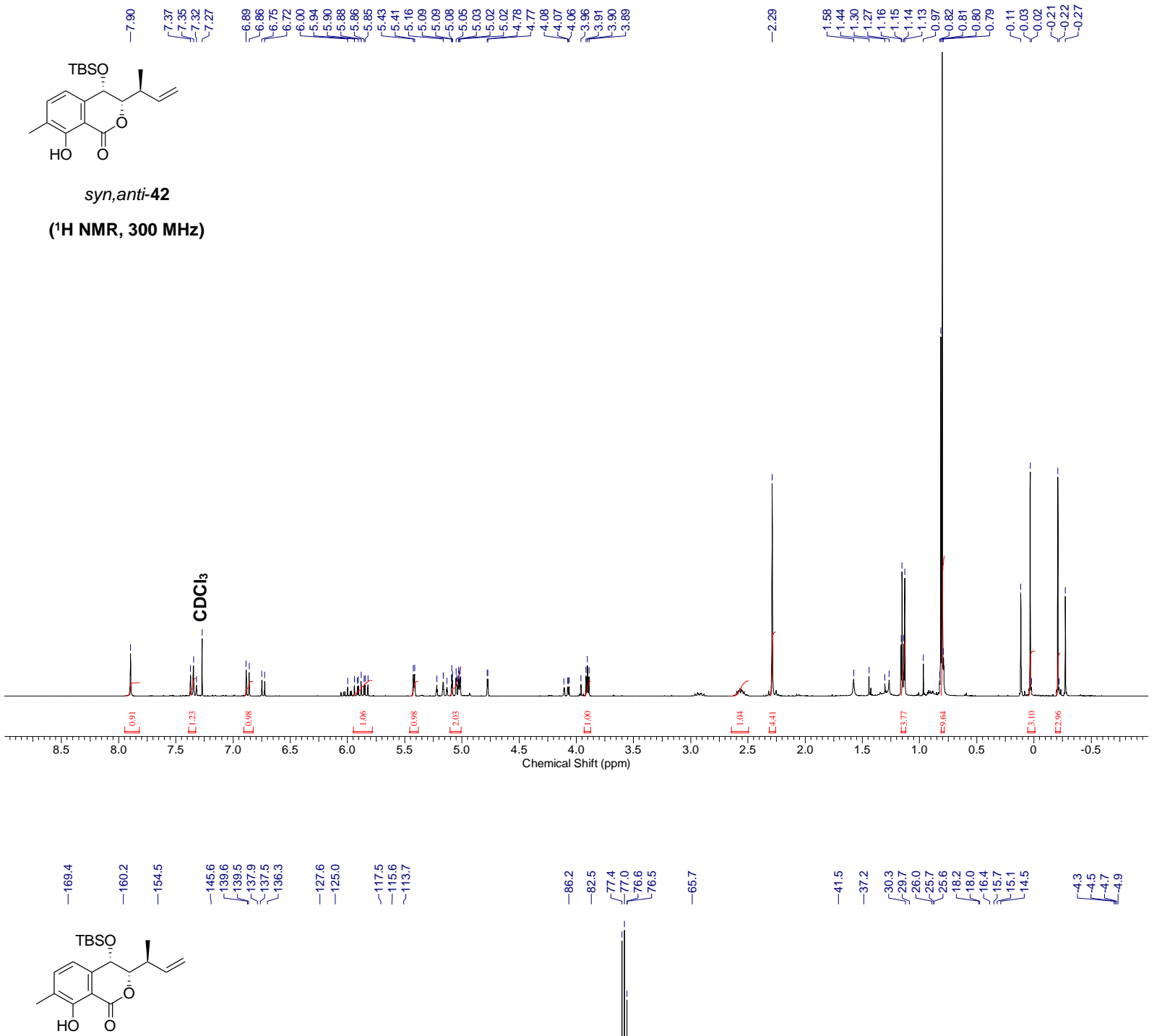

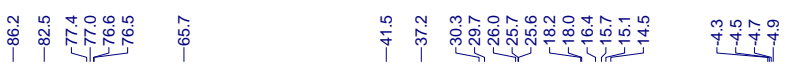

syn,anti-42

( ${ }^{13} \mathrm{C}$ NMR, $\left.75 \mathrm{MHz}\right)$

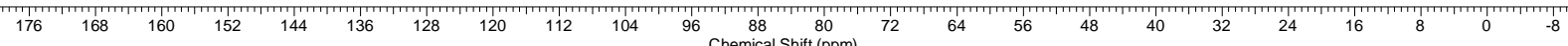




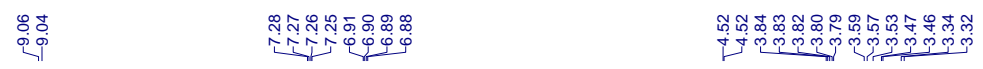

翼

17a

('H NMR, $300 \mathrm{MHz}$ )
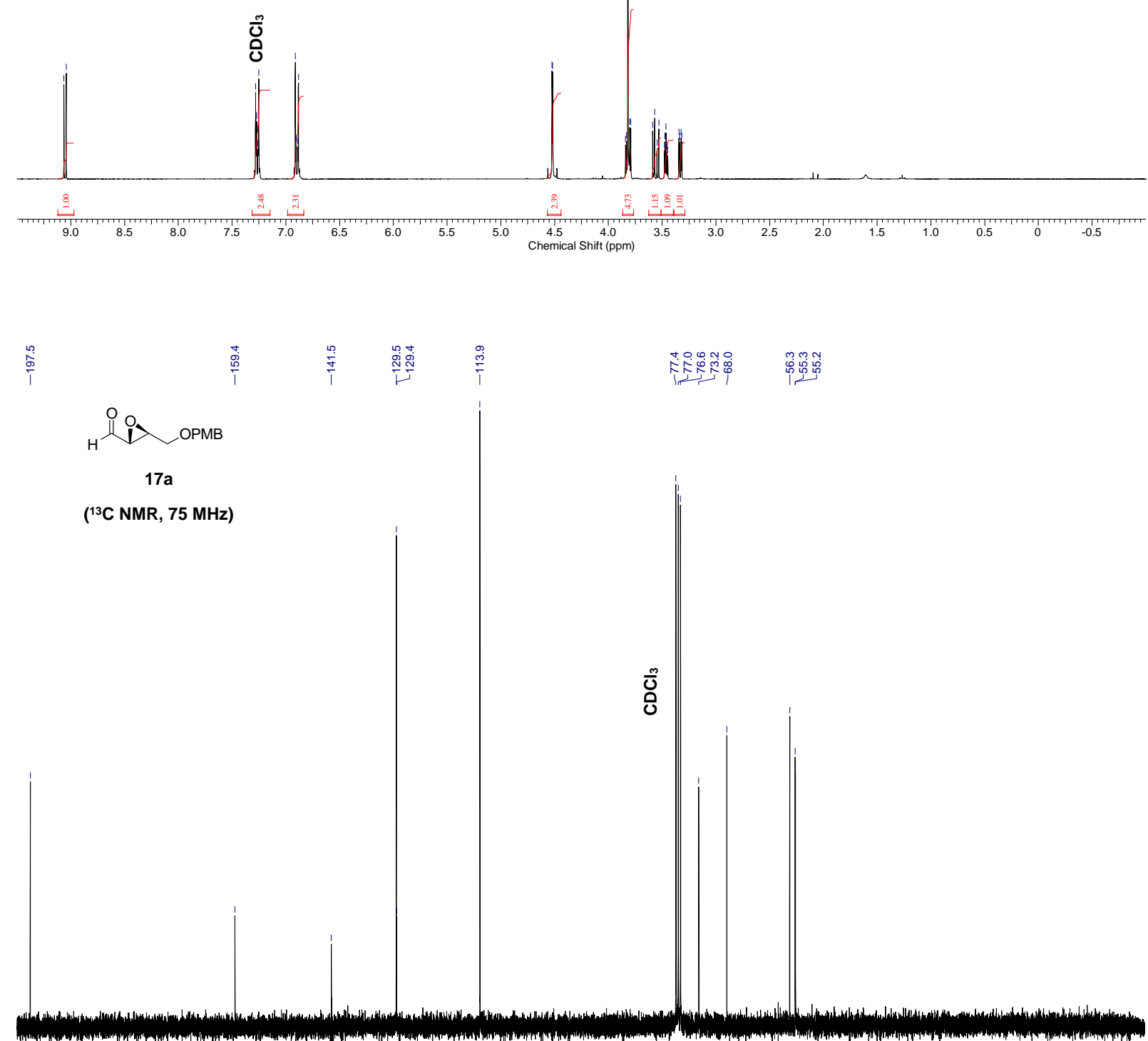

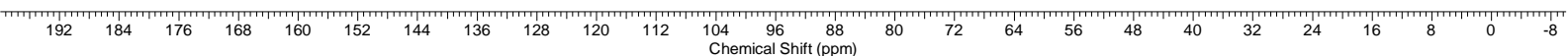


$\overbrace{17 b}^{\stackrel{N}{i}}$

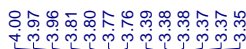

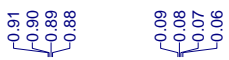

('H NMR, 300 MHz)

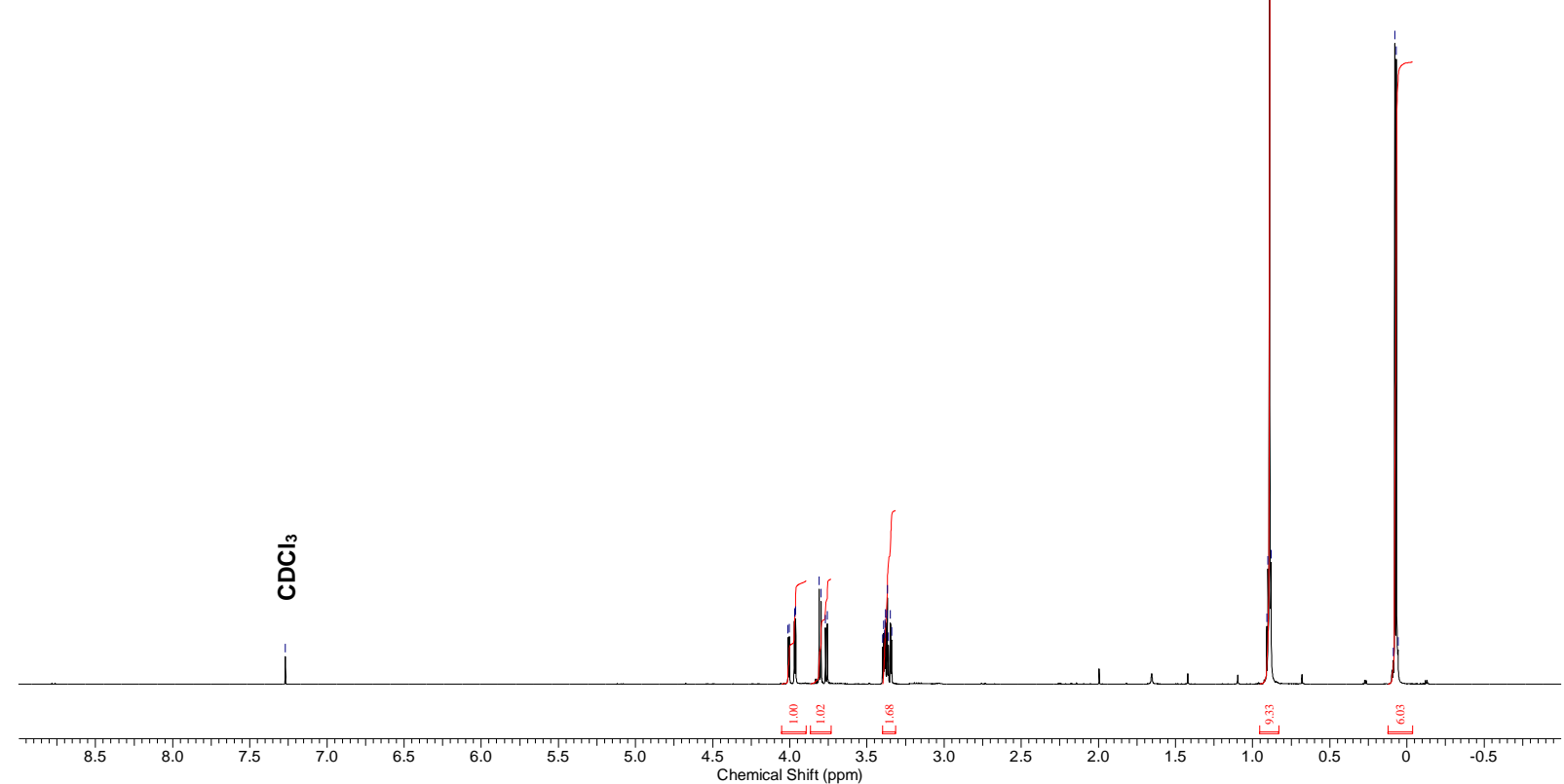

$\underset{\substack{\infty \\ i}}{\stackrel{\infty}{i}}$

$$
\text { 㮍 }
$$

17b

( ${ }^{13} \mathrm{C}$ NMR, $75 \mathrm{MHz}$ )

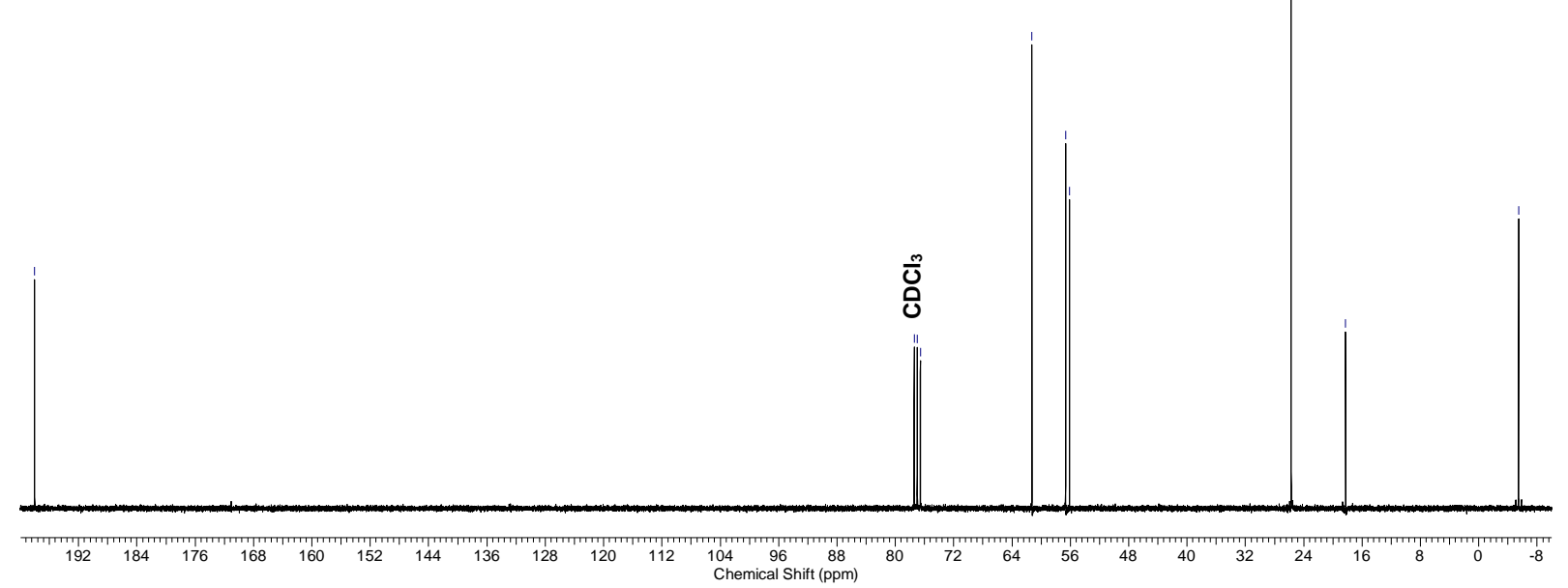




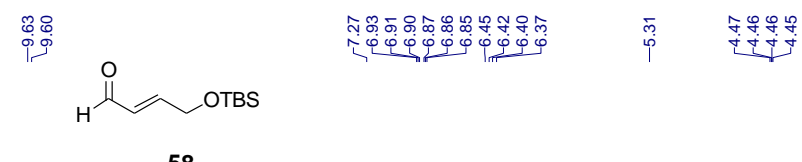

58

('H NMR, $300 \mathrm{MHz}$ )

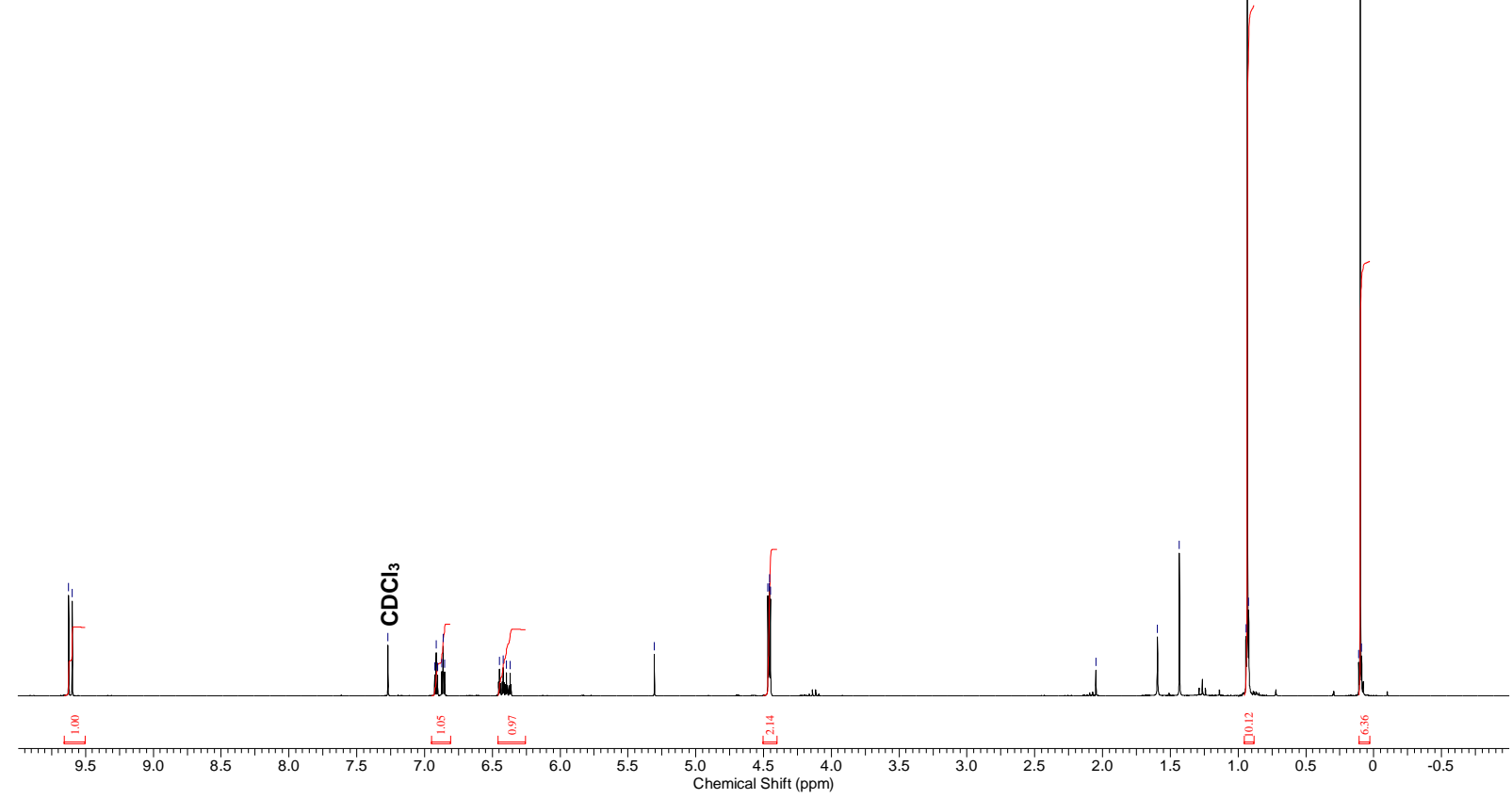

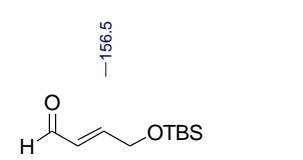

58

( ${ }^{13} \mathrm{C}$ NMR, $75 \mathrm{MHz}$ )

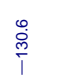<smiles>CCC</smiles>

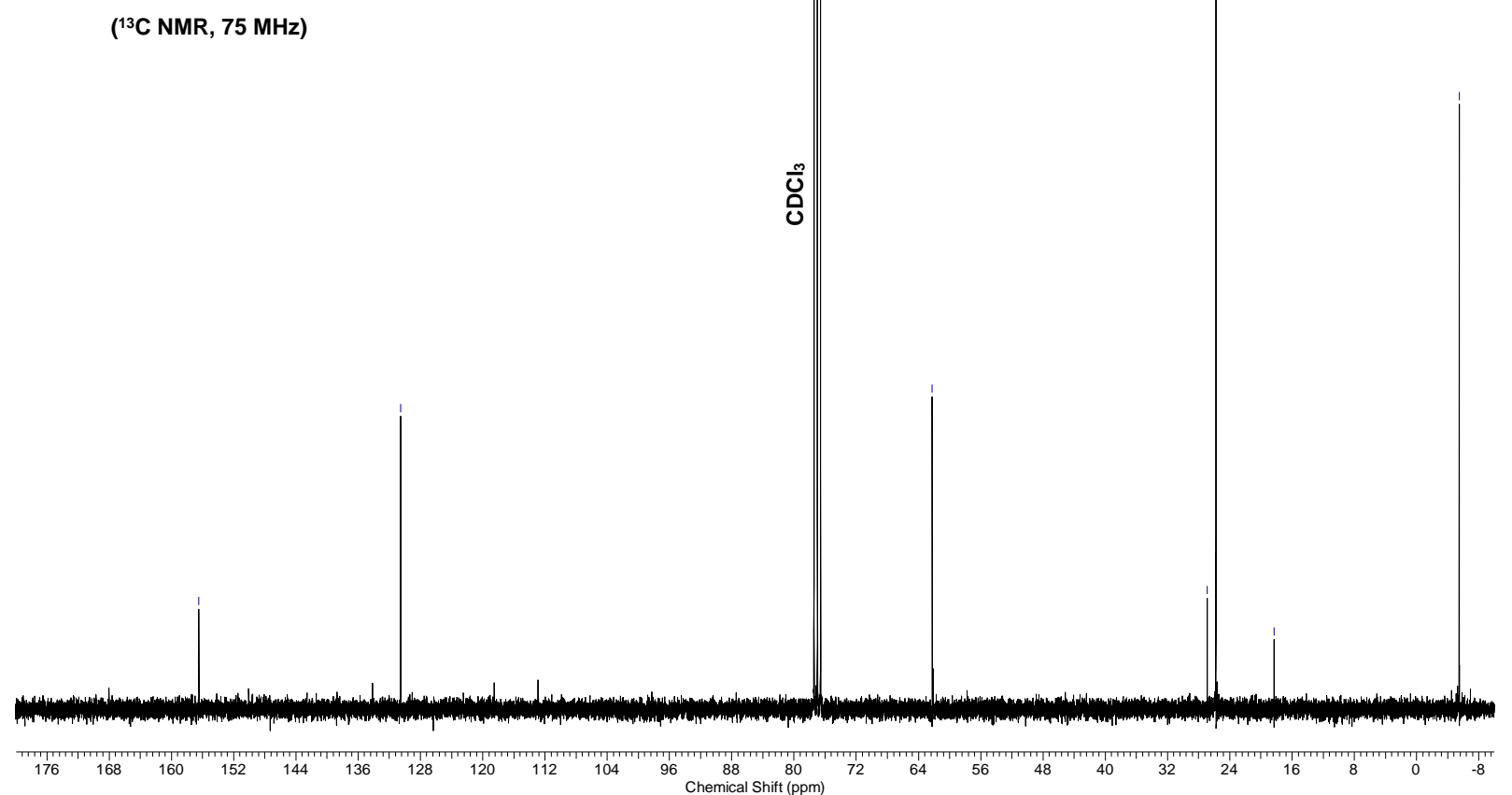




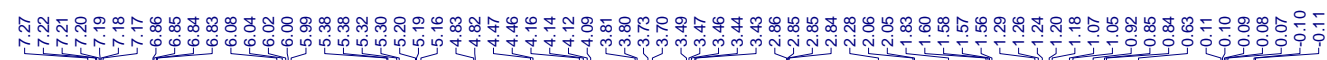

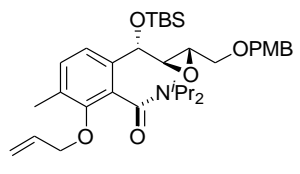

60

('H NMR, $300 \mathrm{MHz}$ )

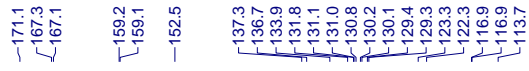

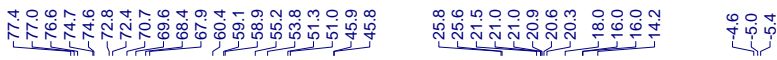

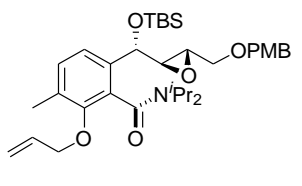

60

( ${ }^{13} \mathrm{C}$ NMR, $75 \mathrm{MHz}$ )
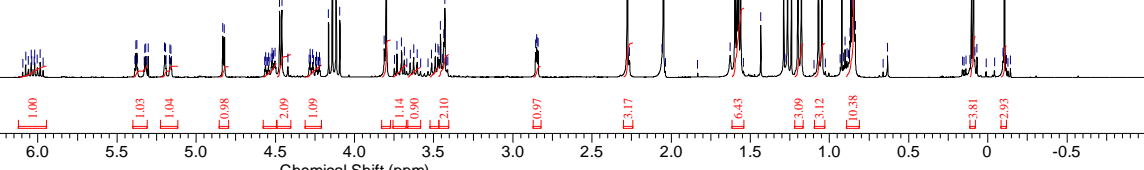

ชั0

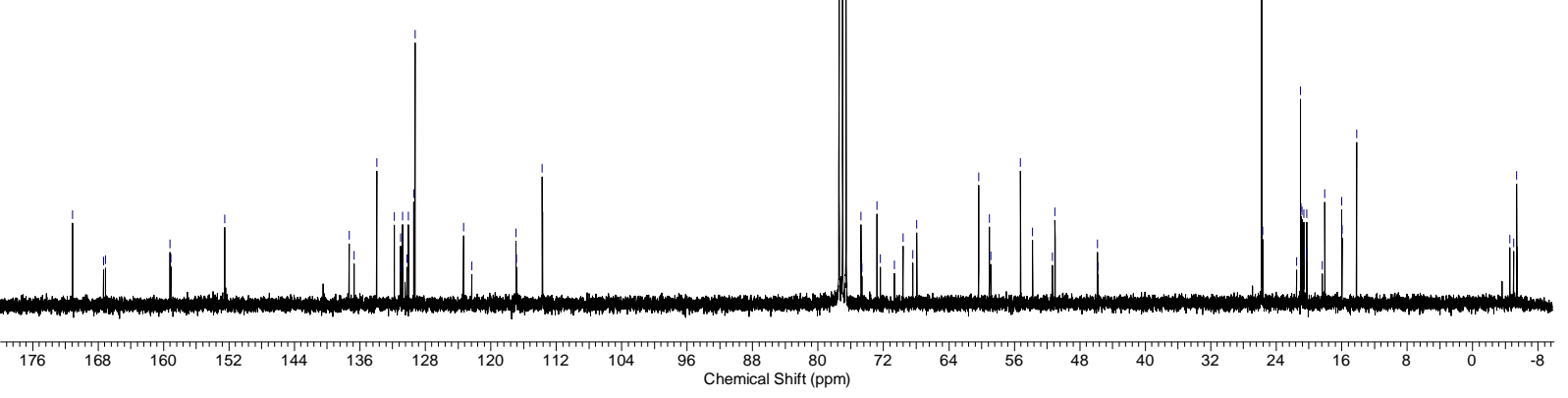


용ㅇㅇ

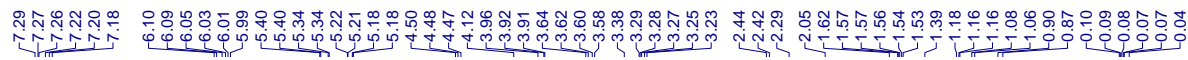

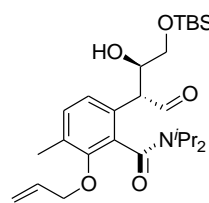

62

( ${ }^{\mathrm{H}} \mathrm{NMR}, 300 \mathrm{MHz}$ )

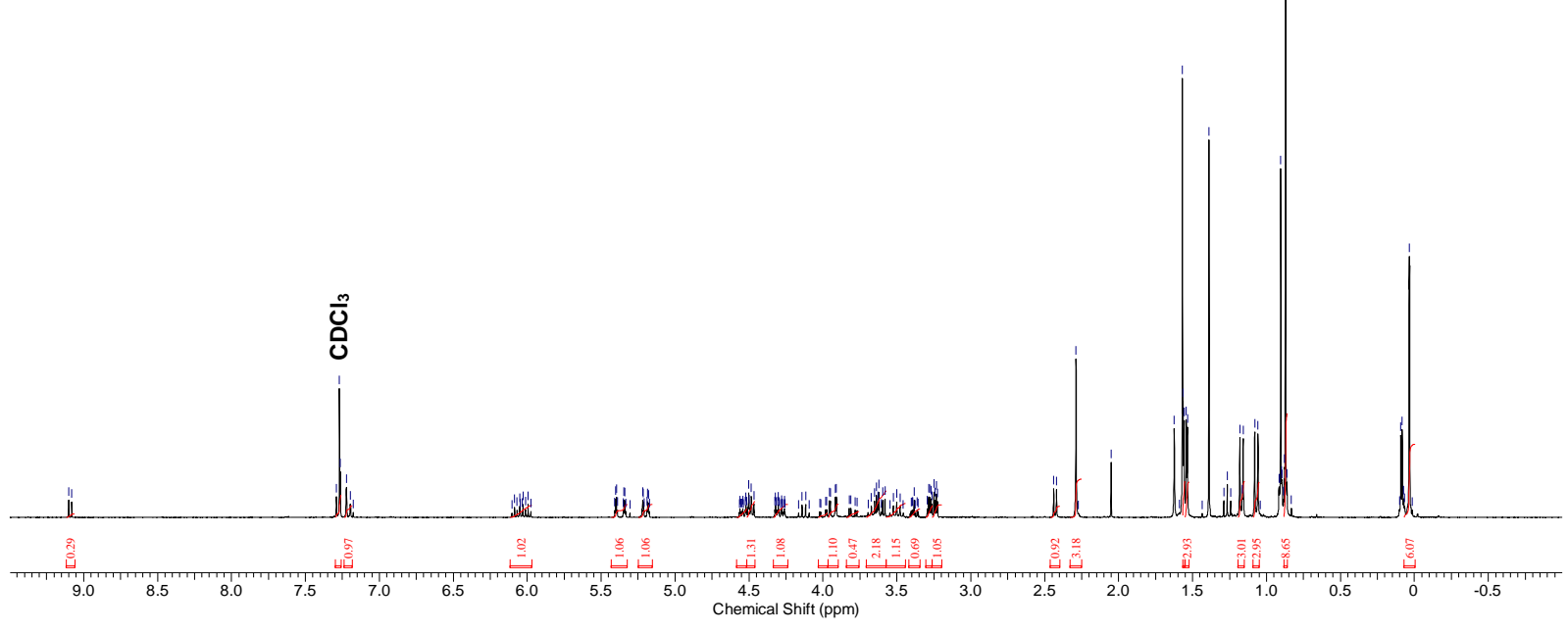

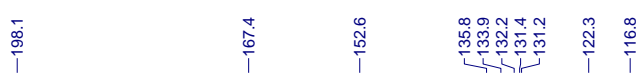

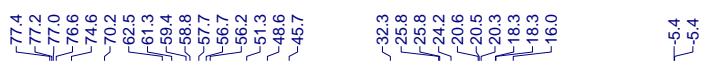

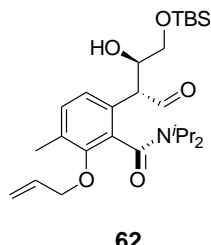

융

( ${ }^{13} \mathrm{C}$ NMR, $75 \mathrm{MHz}$ )

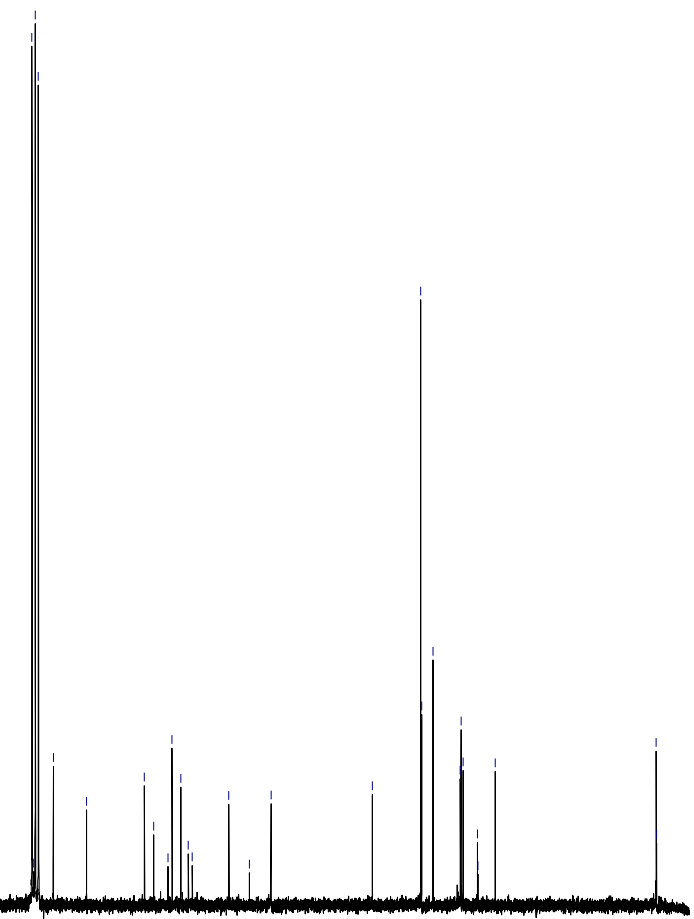

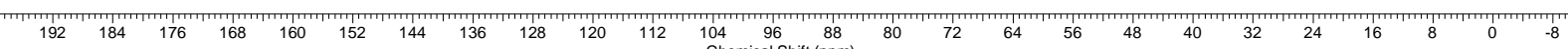




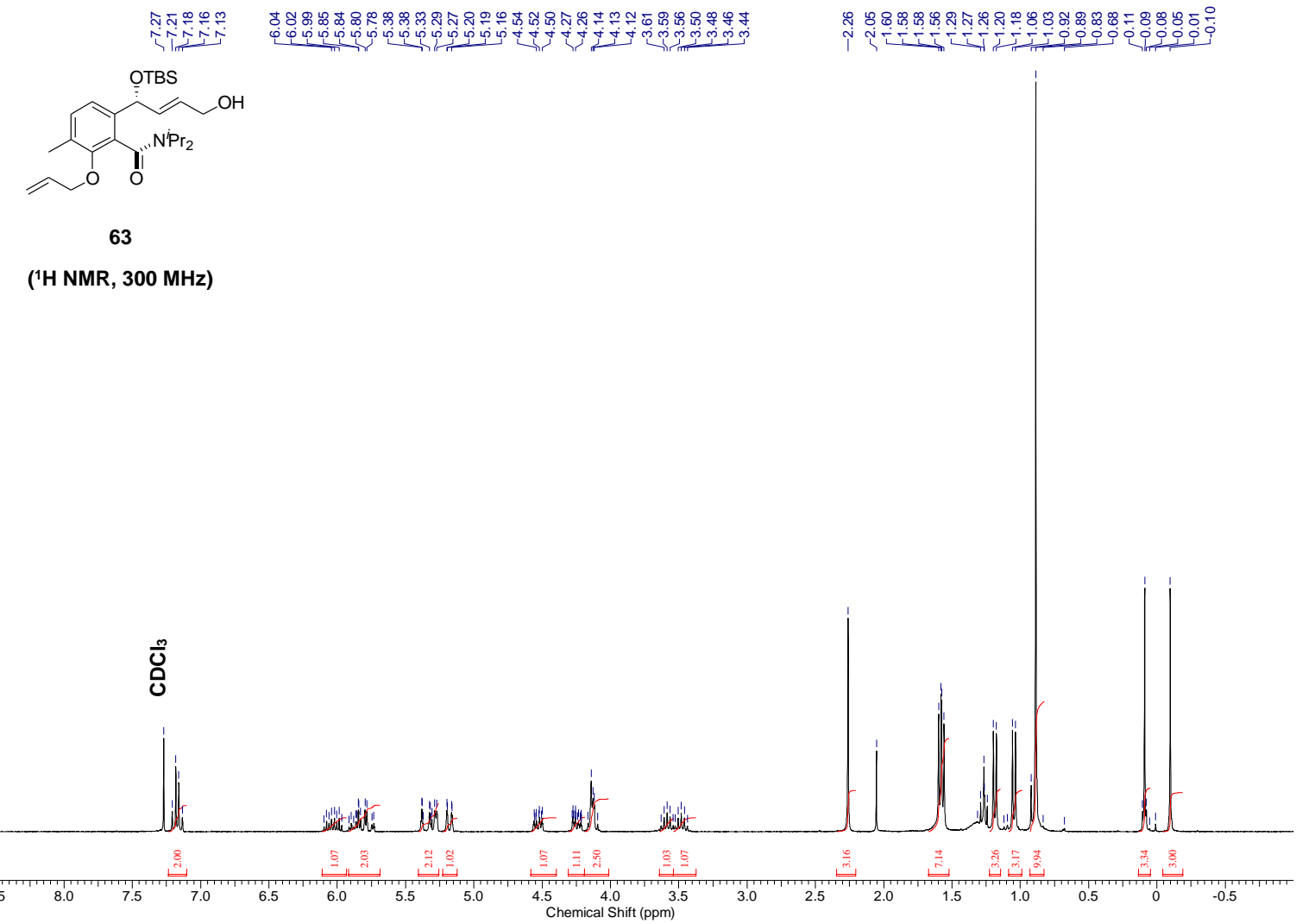

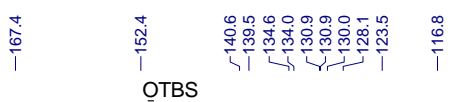

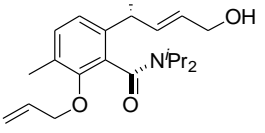

63

( ${ }^{13} \mathrm{C}$ NMR, $\left.75 \mathrm{MHz}\right)$

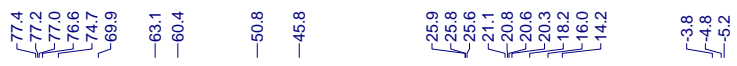

\%

Oั

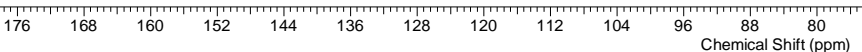




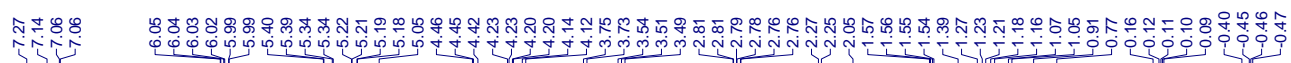

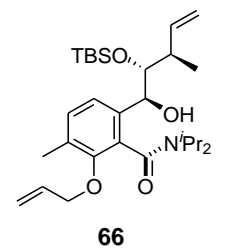

( ${ }^{1} \mathrm{H}$ NMR, $300 \mathrm{MHz}$ )

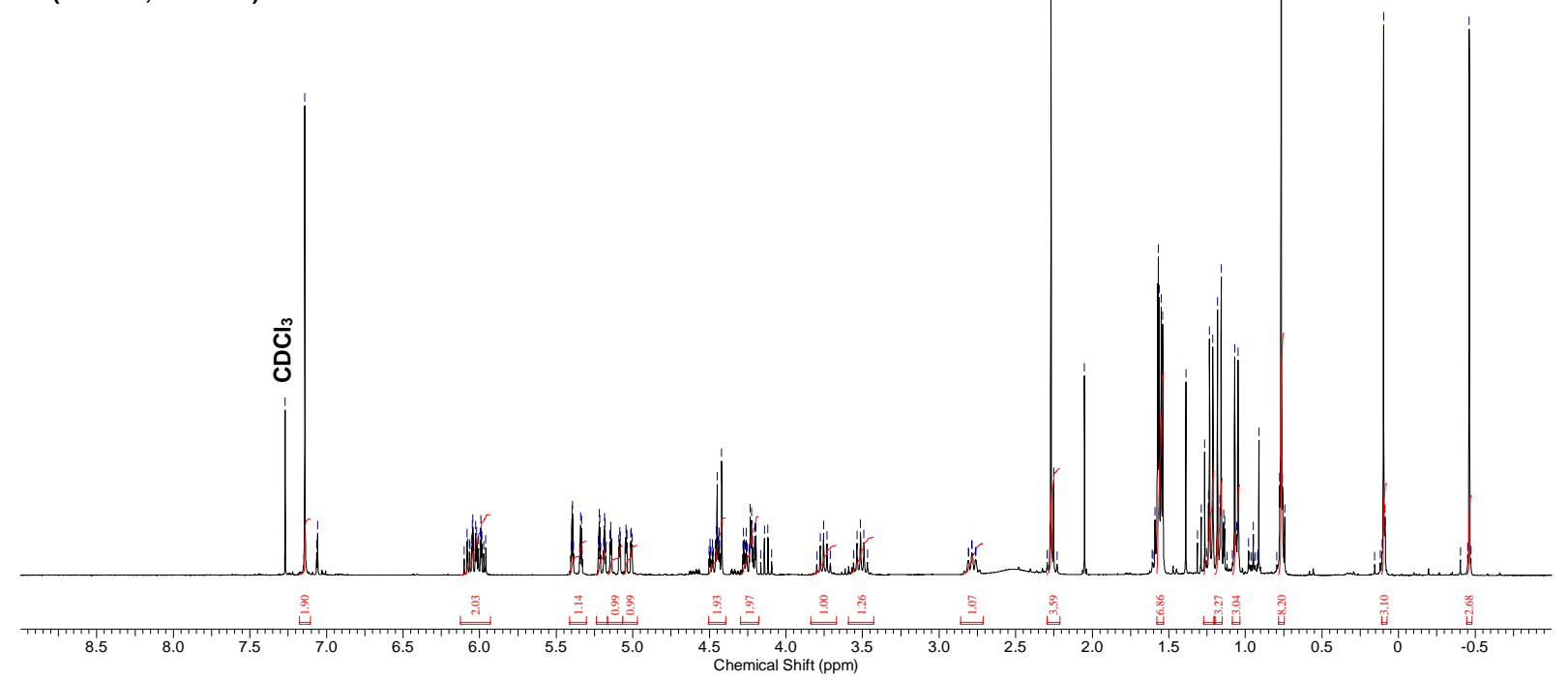

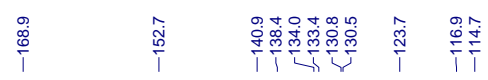

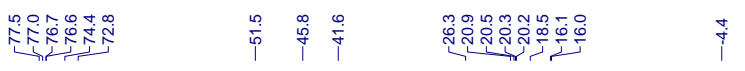

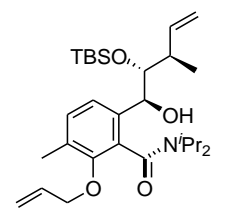

66

( ${ }^{13} \mathrm{C}$ NMR, $\left.75 \mathrm{MHz}\right)$

ֻั0

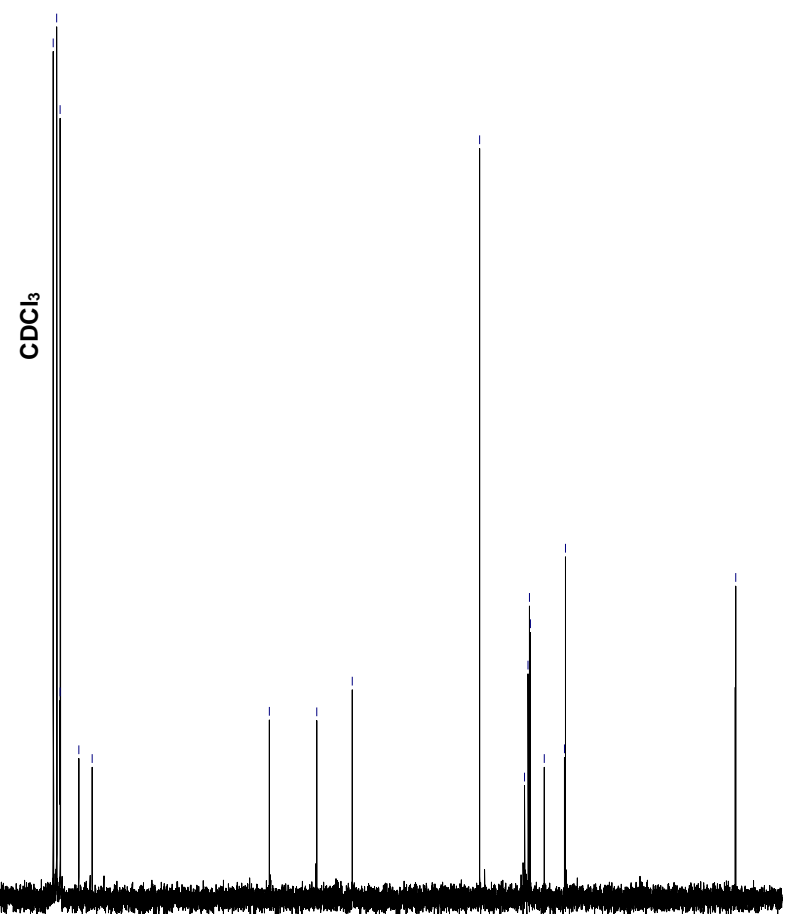

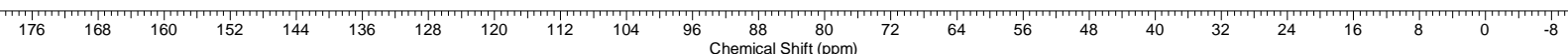




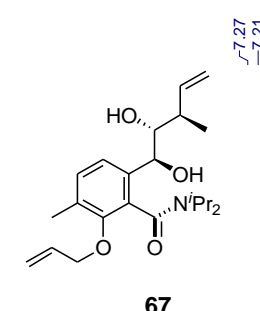

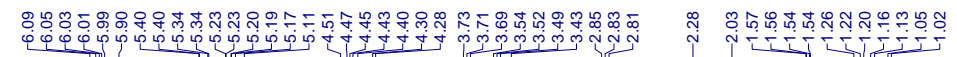

('H NMR, $300 \mathrm{MHz}$ )

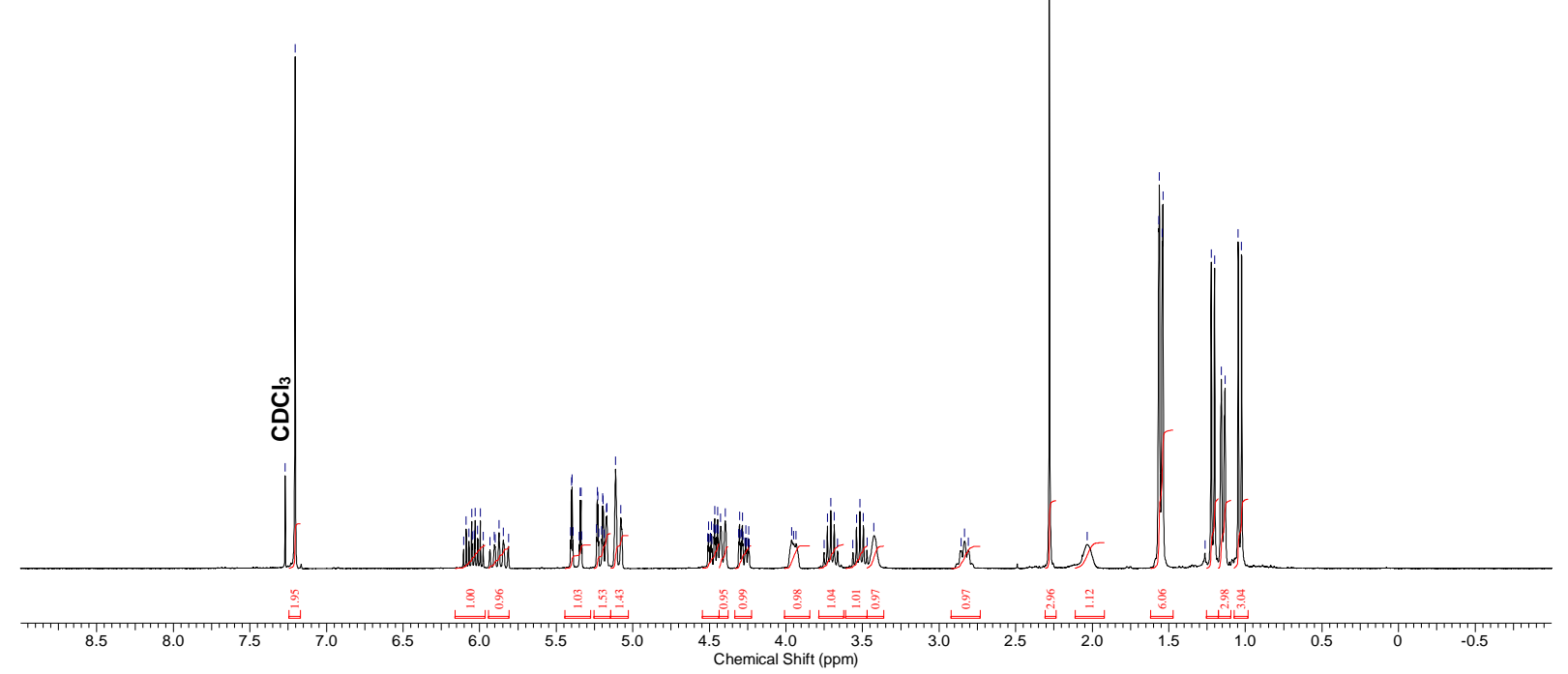

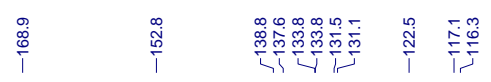

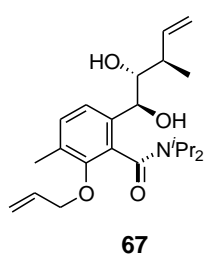

( ${ }^{13} \mathrm{C}$ NMR, $\left.125 \mathrm{MHz}\right)$

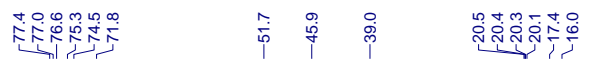

ชั

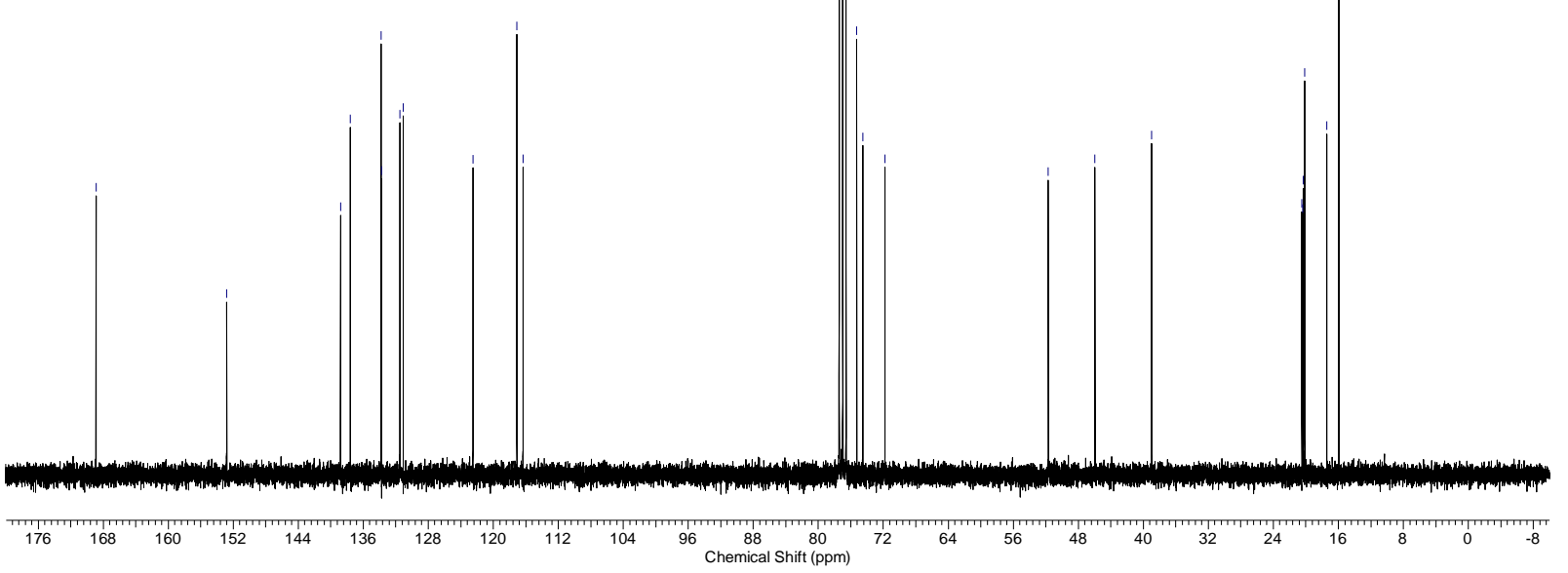




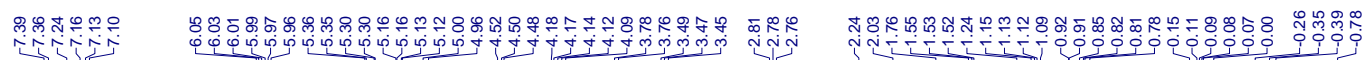

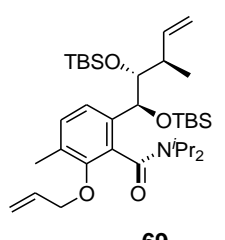

69

('H NMR, $300 \mathrm{MHz}$ )
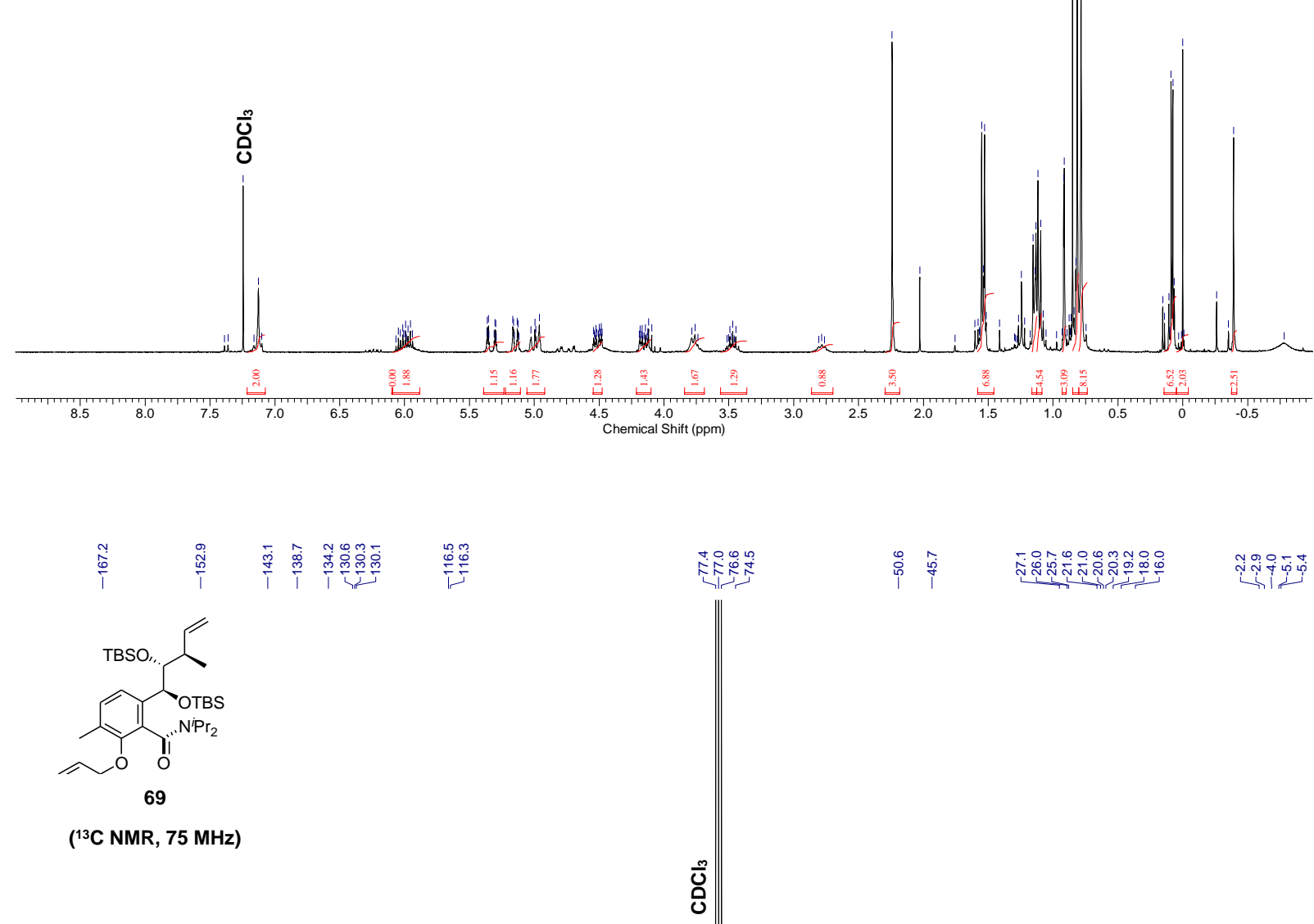

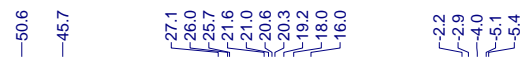

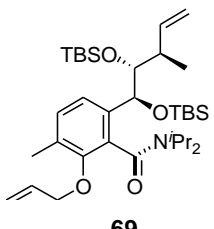

$\left({ }^{13} \mathrm{C}\right.$ NMR, $\left.75 \mathrm{MHz}\right)$

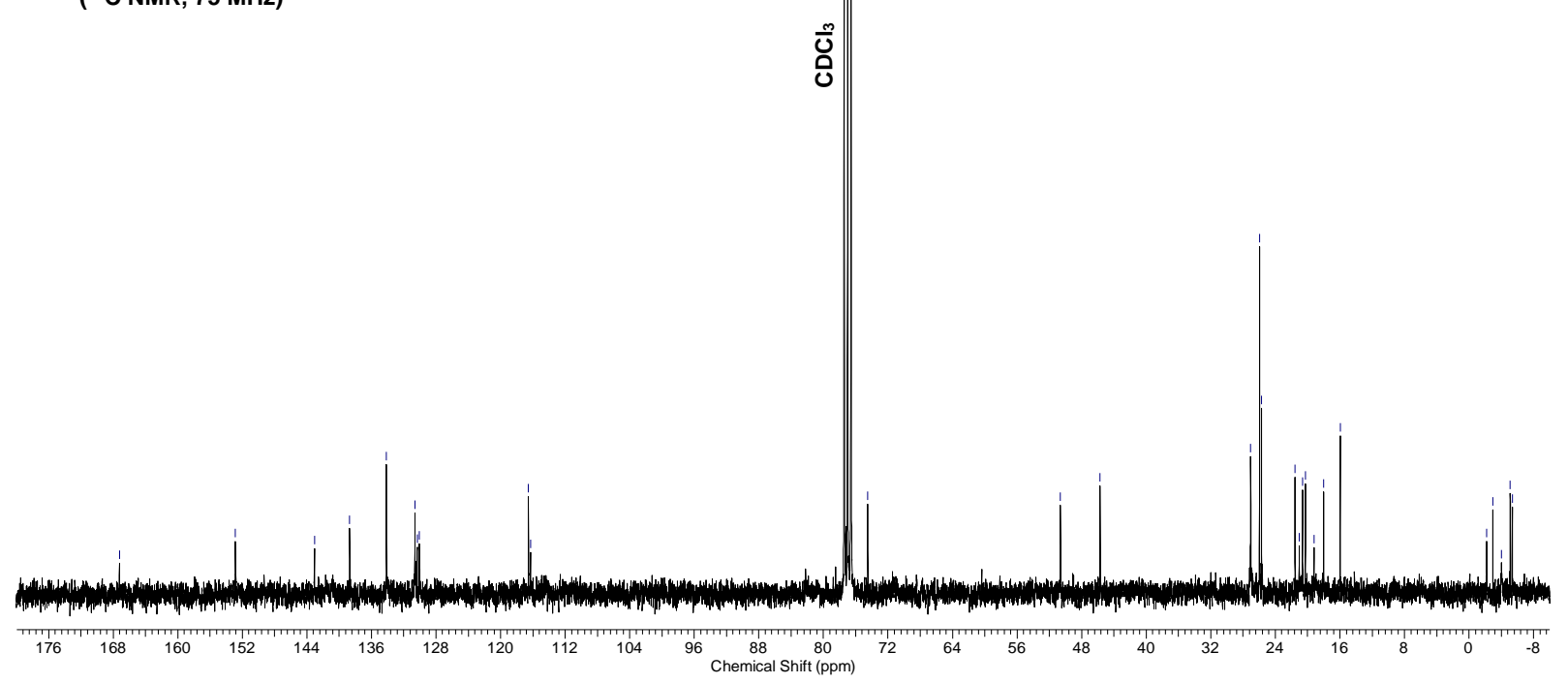




ETO $\underbrace{\overbrace{33 \mathrm{~b}}}_{\text {OTES }}$

('H NMR, 300 MHz)

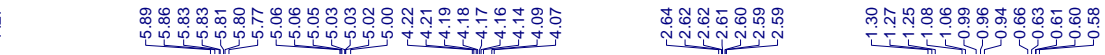
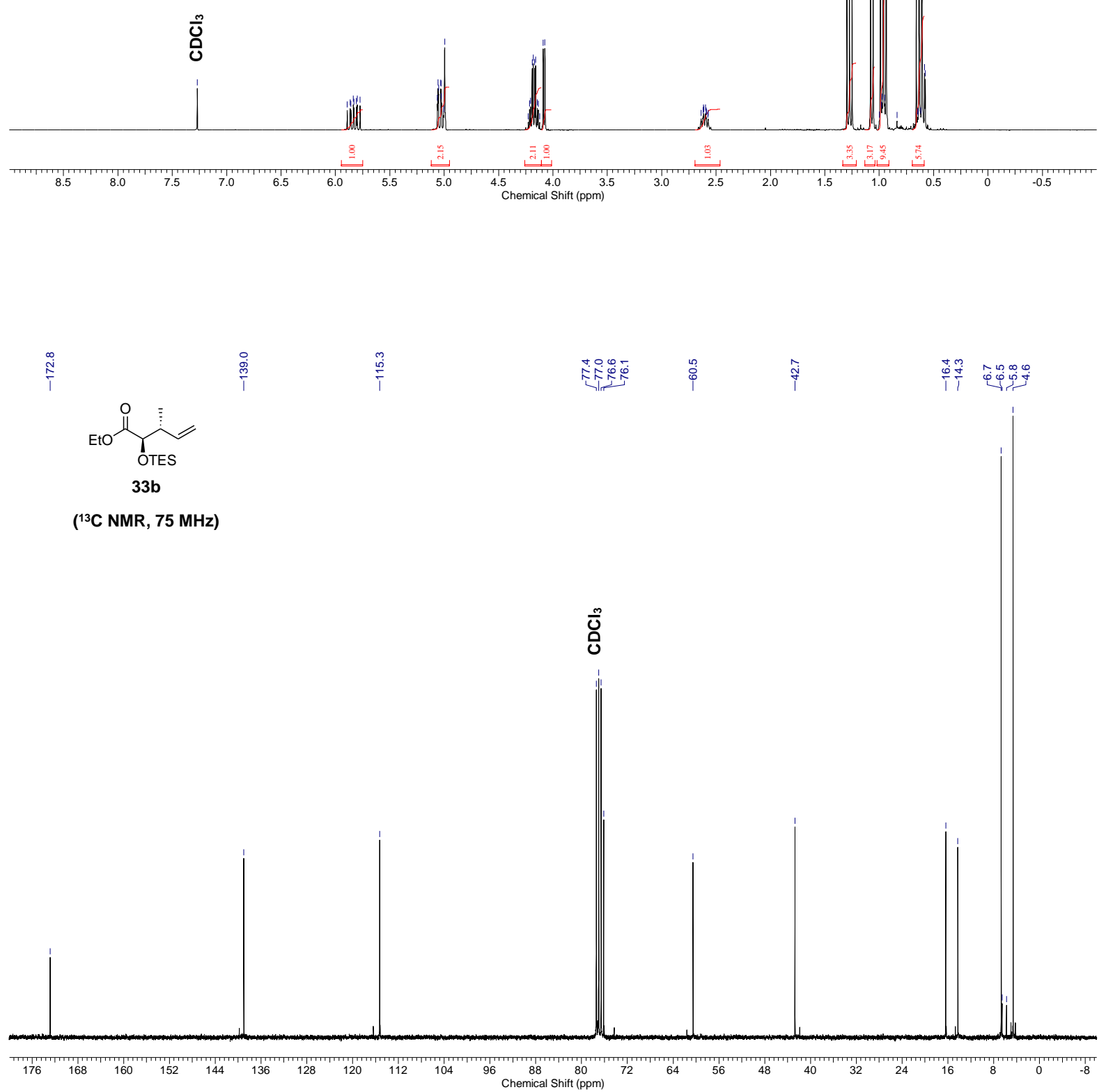


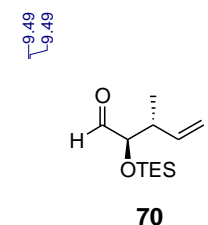

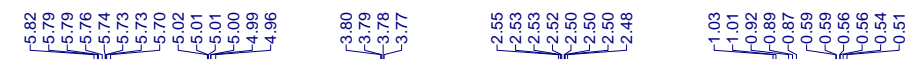

('H NMR, $300 \mathrm{MHz}$ )

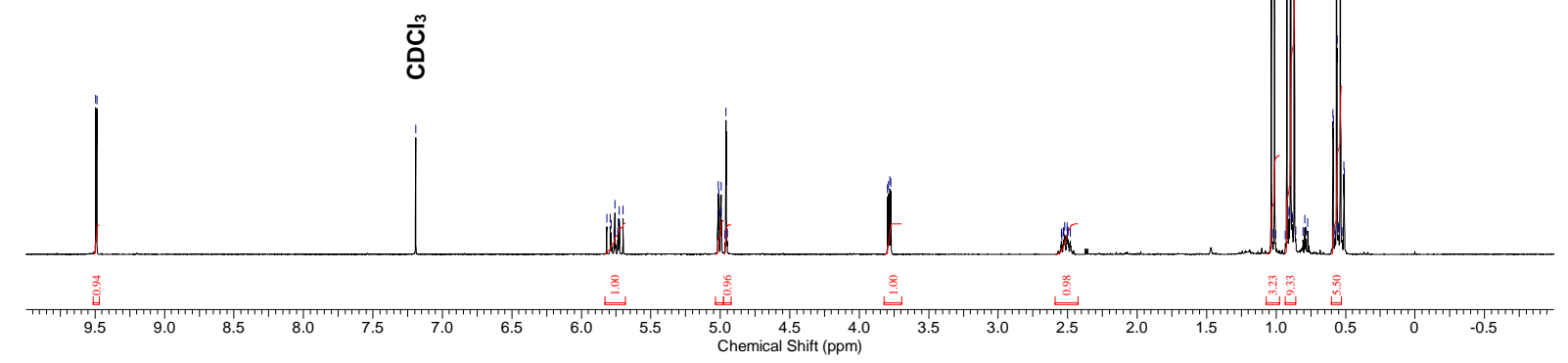

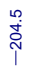

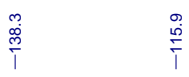

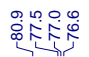

$\stackrel{+}{\dot{y}}$

$\underbrace{\text { ON }}_{\text {OTES }}$

$\stackrel{\circ}{\stackrel{0}{\not}}$

70

$\left({ }^{13} \mathrm{C}\right.$ NMR, $\left.75 \mathrm{MHz}\right)$

ठั๊

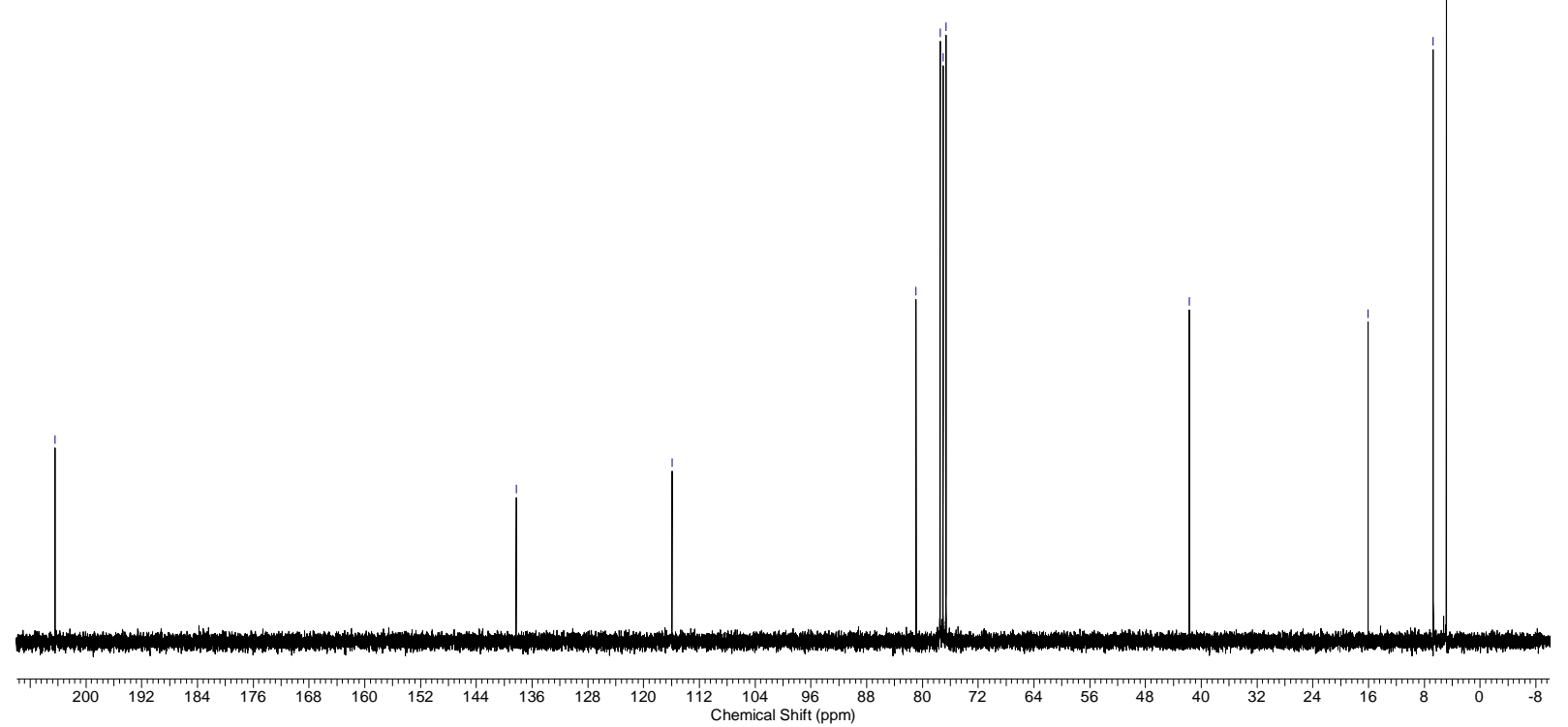




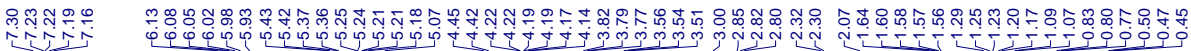

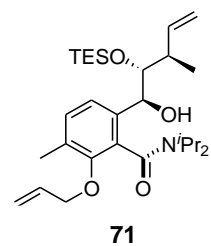

('H NMR, $300 \mathrm{MHz}$ )

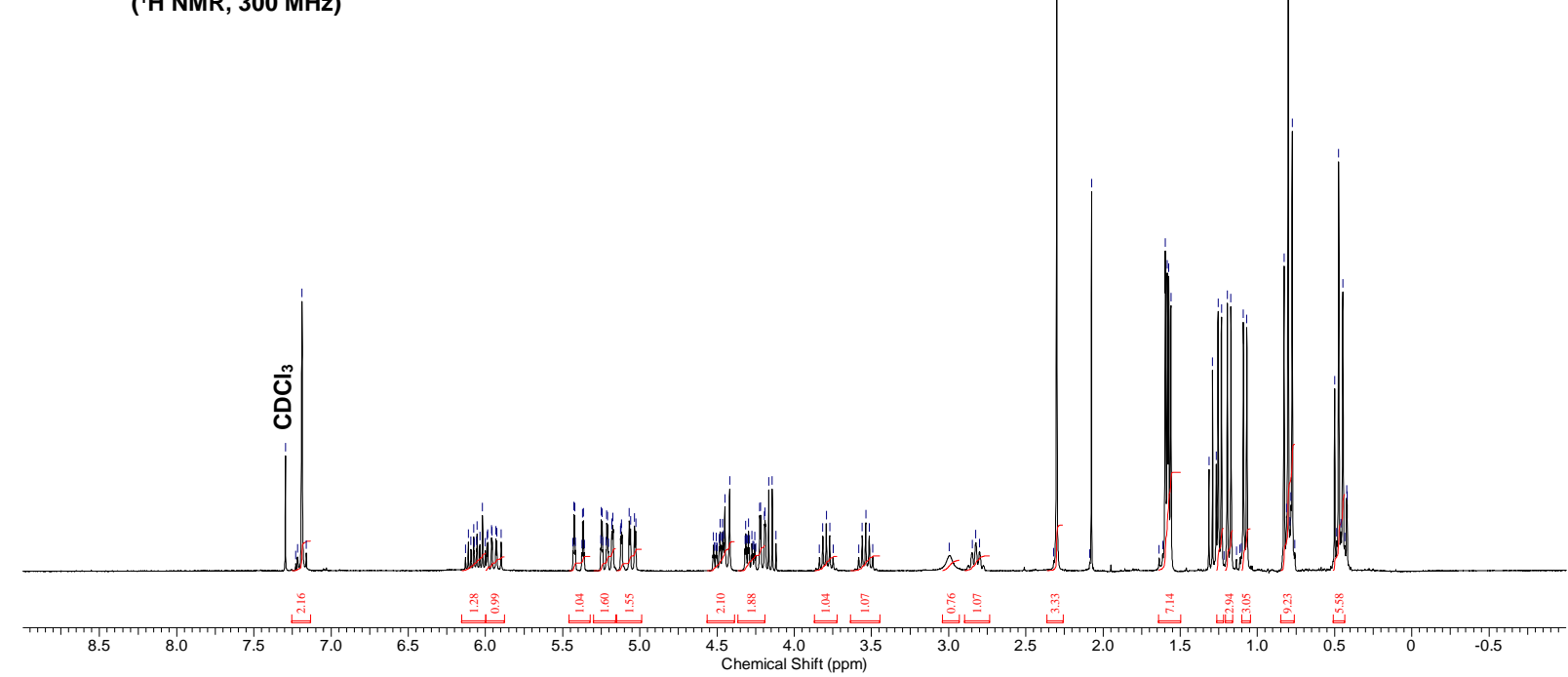

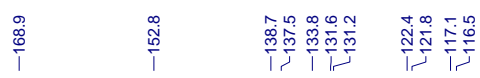

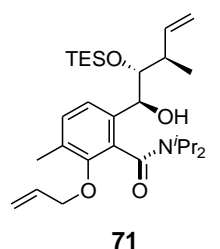

$\left({ }^{13} \mathrm{C} \mathrm{NMR}, 75 \mathrm{MHz}\right)$

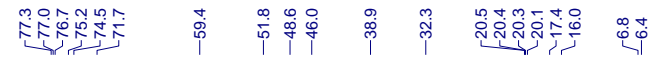

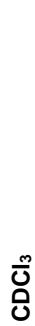

윰

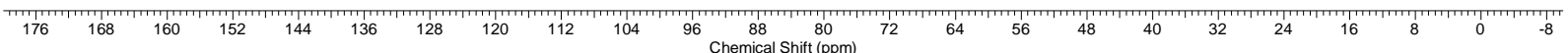




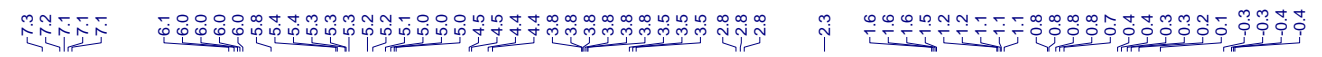

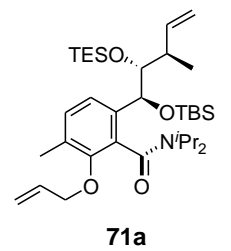

('H NMR, $300 \mathrm{MHz}$ )
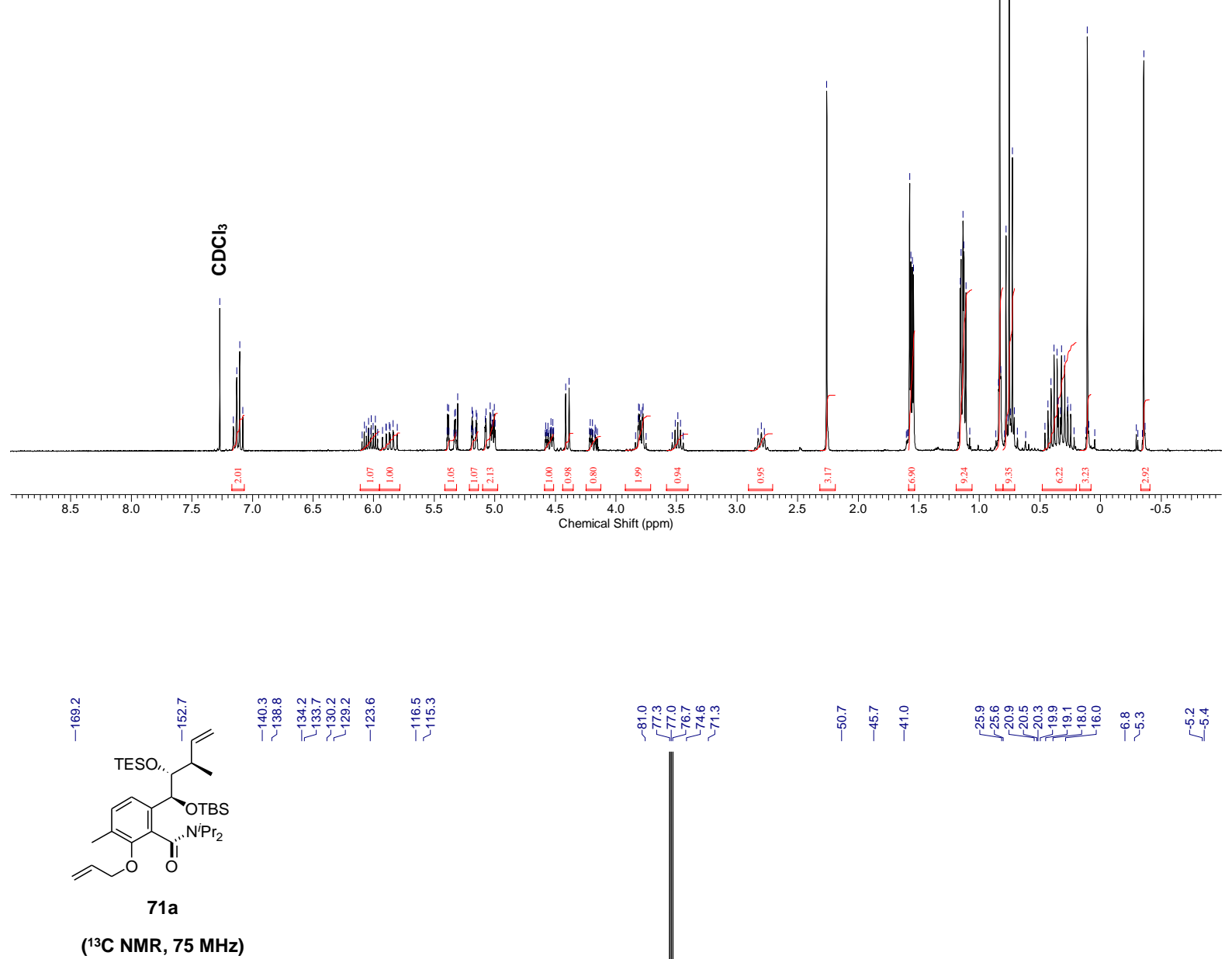

$\left({ }^{13} \mathrm{C}\right.$ NMR, $\left.75 \mathrm{MHz}\right)$

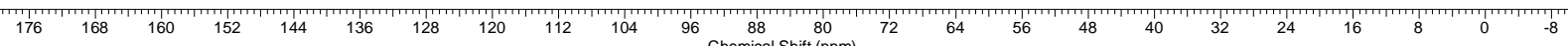




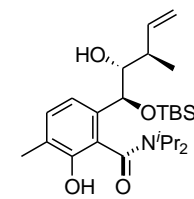

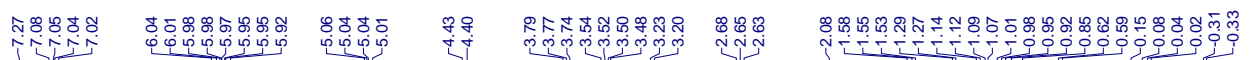

41

('H NMR, $300 \mathrm{MHz})$

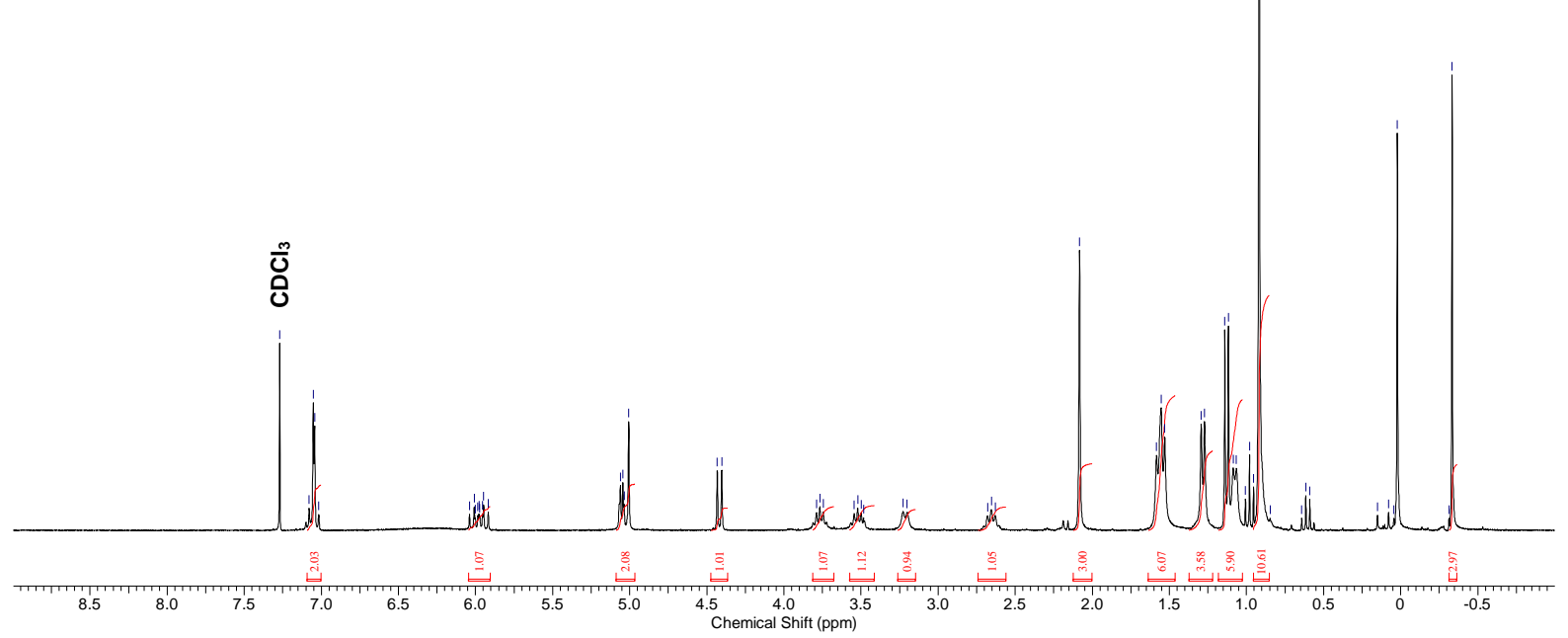

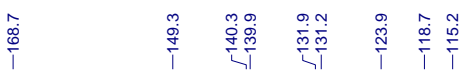

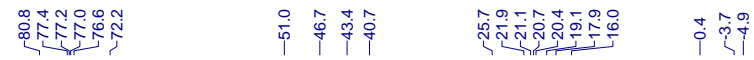

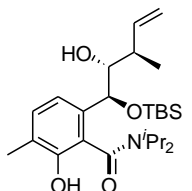

41

( ${ }^{13} \mathrm{C}$ NMR, $75 \mathrm{MHz}$ )

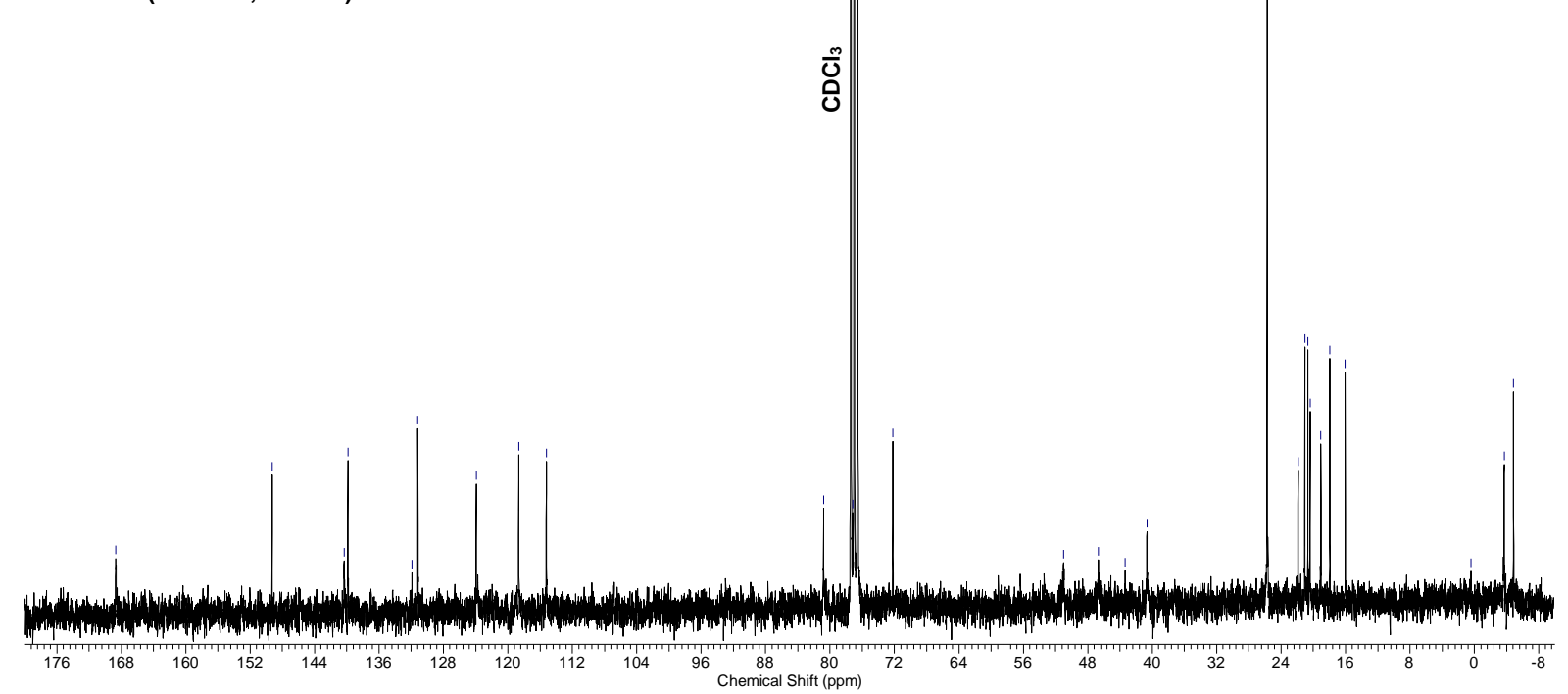


$\stackrel{\infty}{\stackrel{\infty}{T}}$

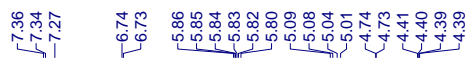

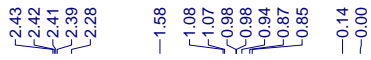

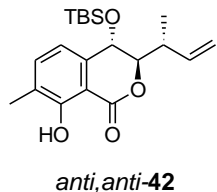

('H NMR, 600 MHz)

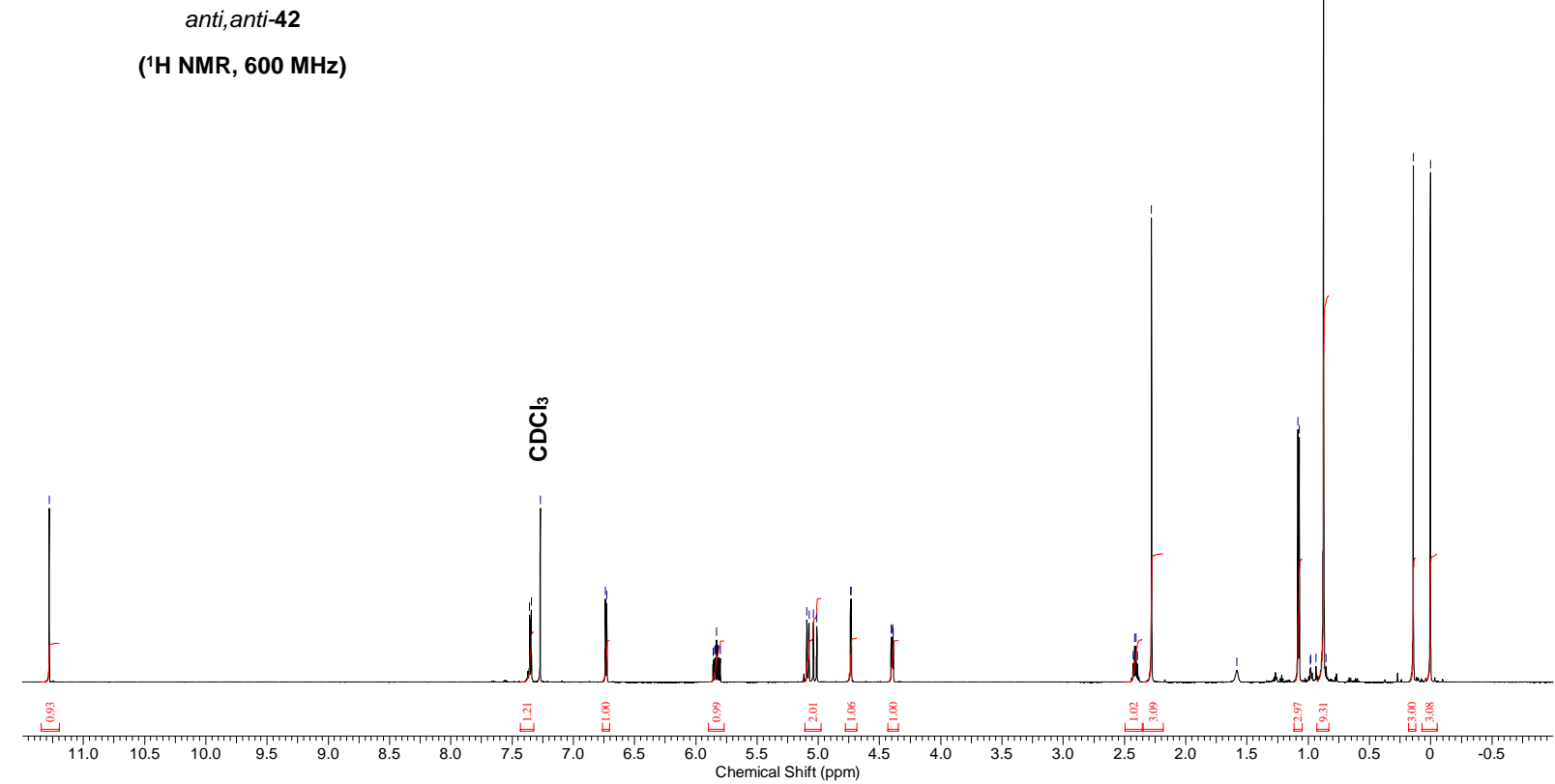

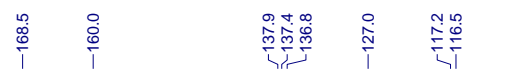

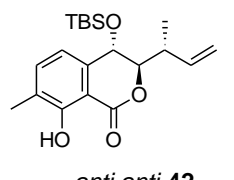

anti,anti-42

( ${ }^{13} \mathrm{C}$ NMR, $\left.151 \mathrm{MHz}\right)$

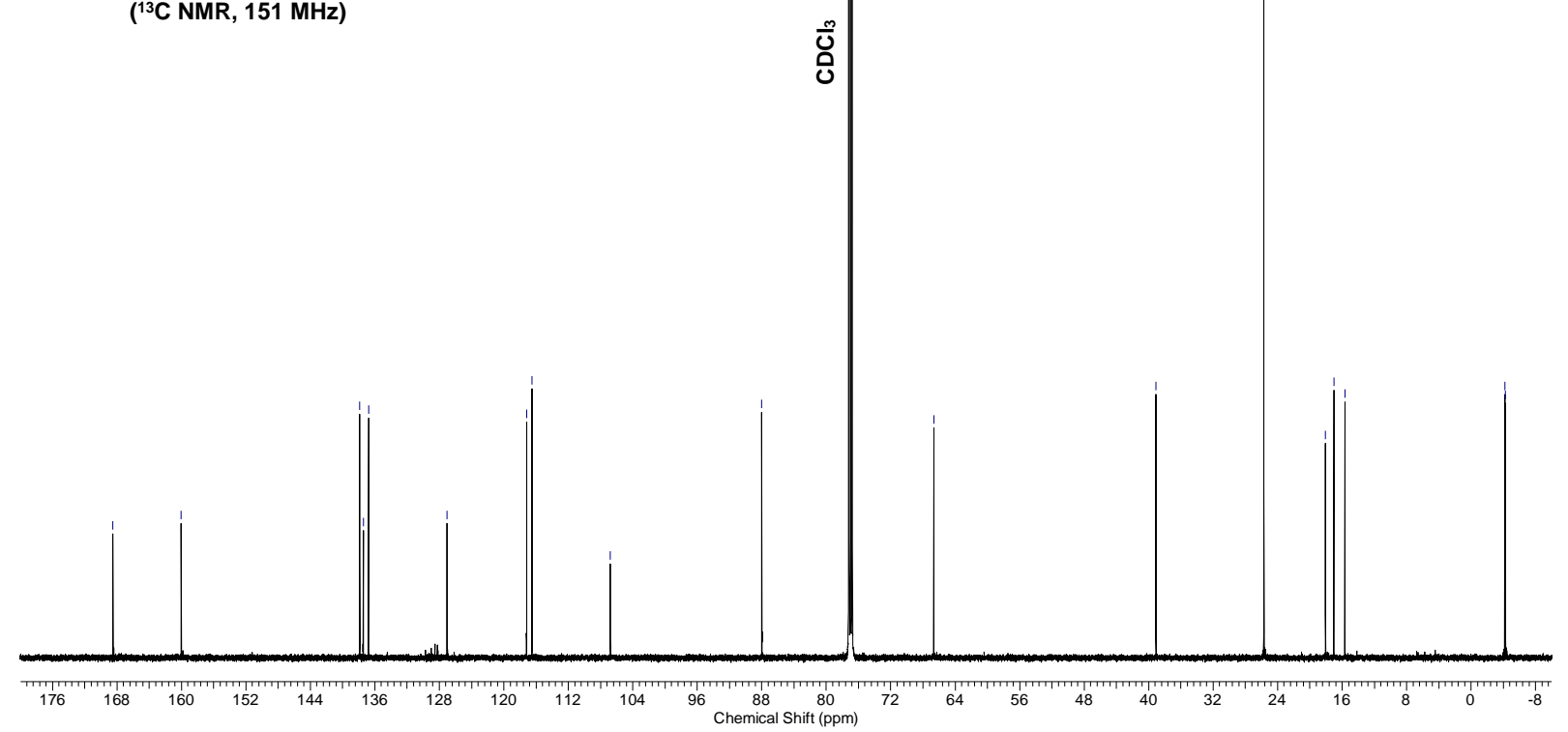


总婴婴

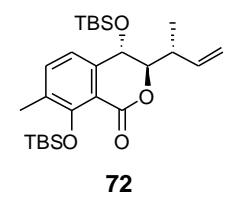

('H NMR, $300 \mathrm{MHz}$ )

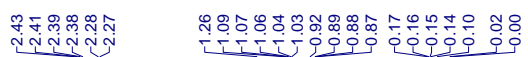

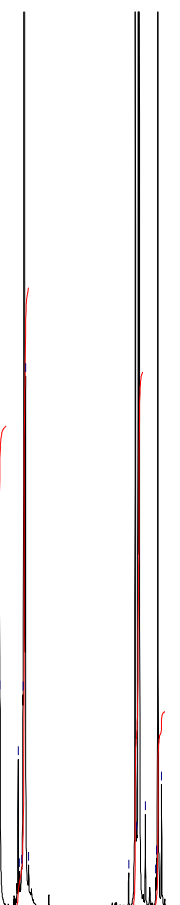

อ 5

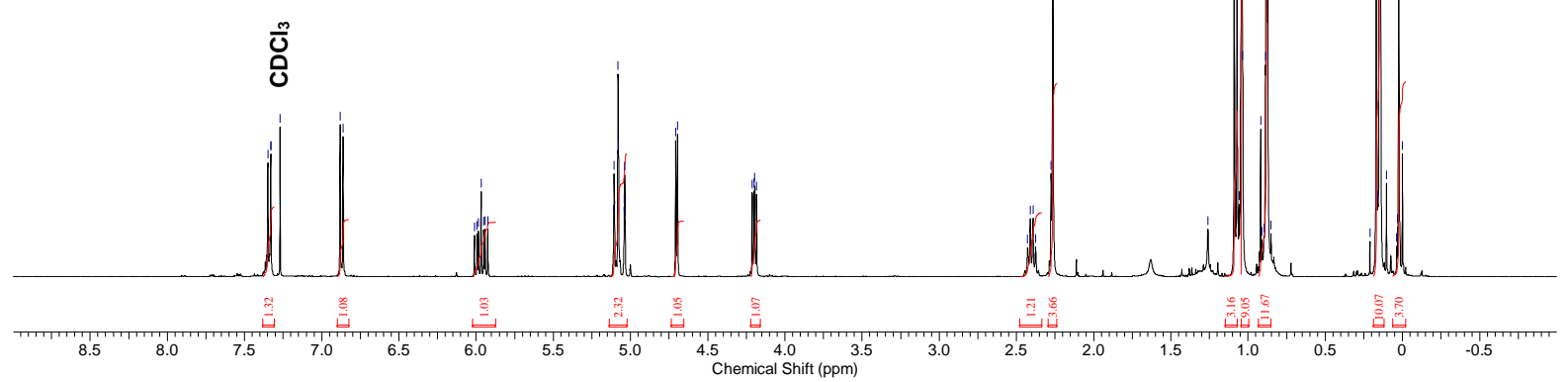

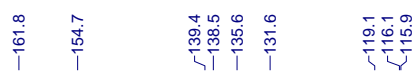

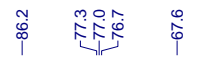

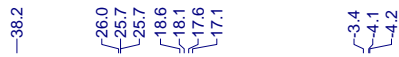

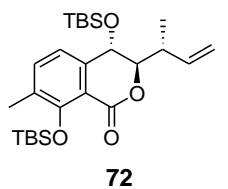

( ${ }^{13} \mathrm{C}$ NMR, $\left.75 \mathrm{MHz}\right)$

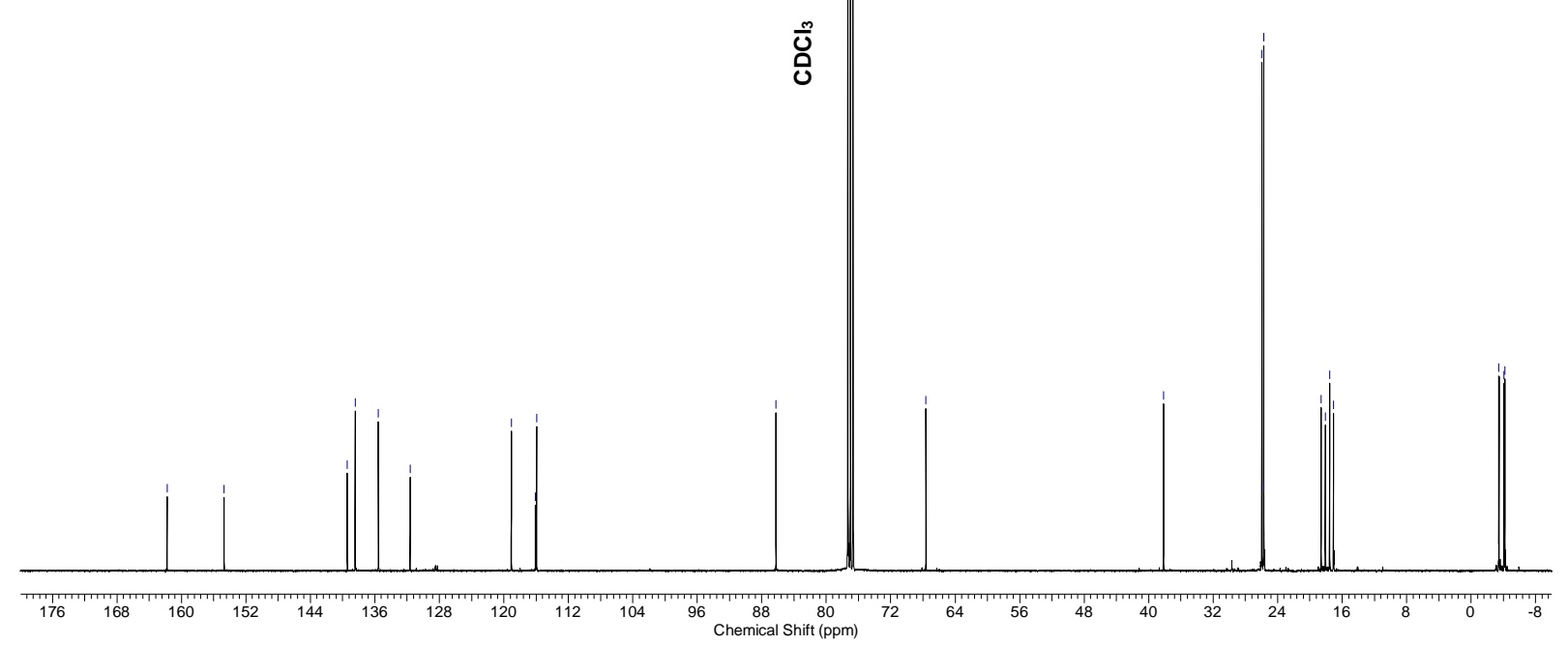




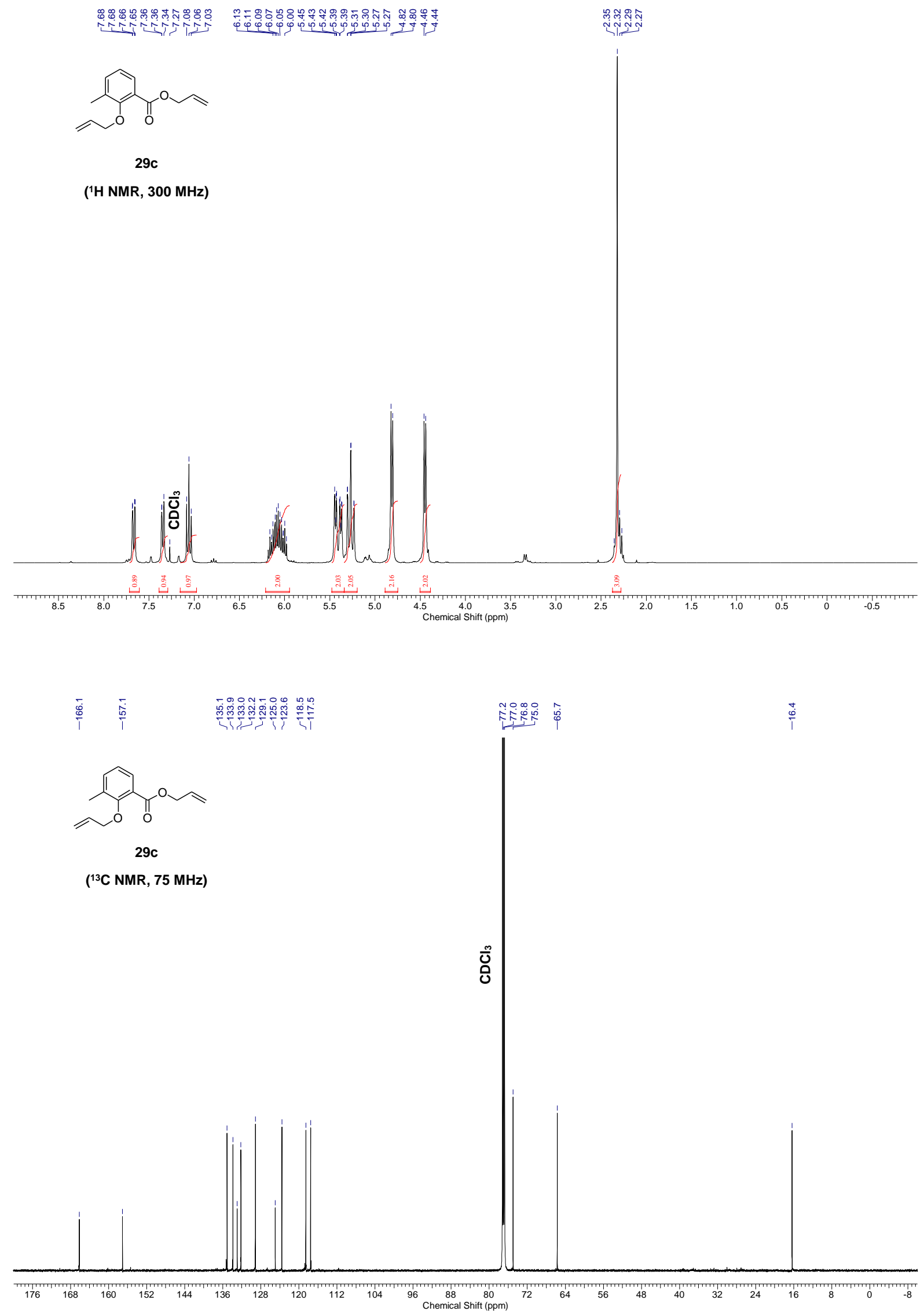




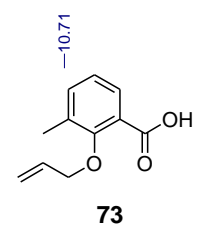

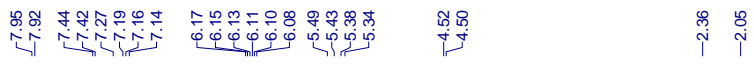

('H NMR, $300 \mathrm{MHz}$ )

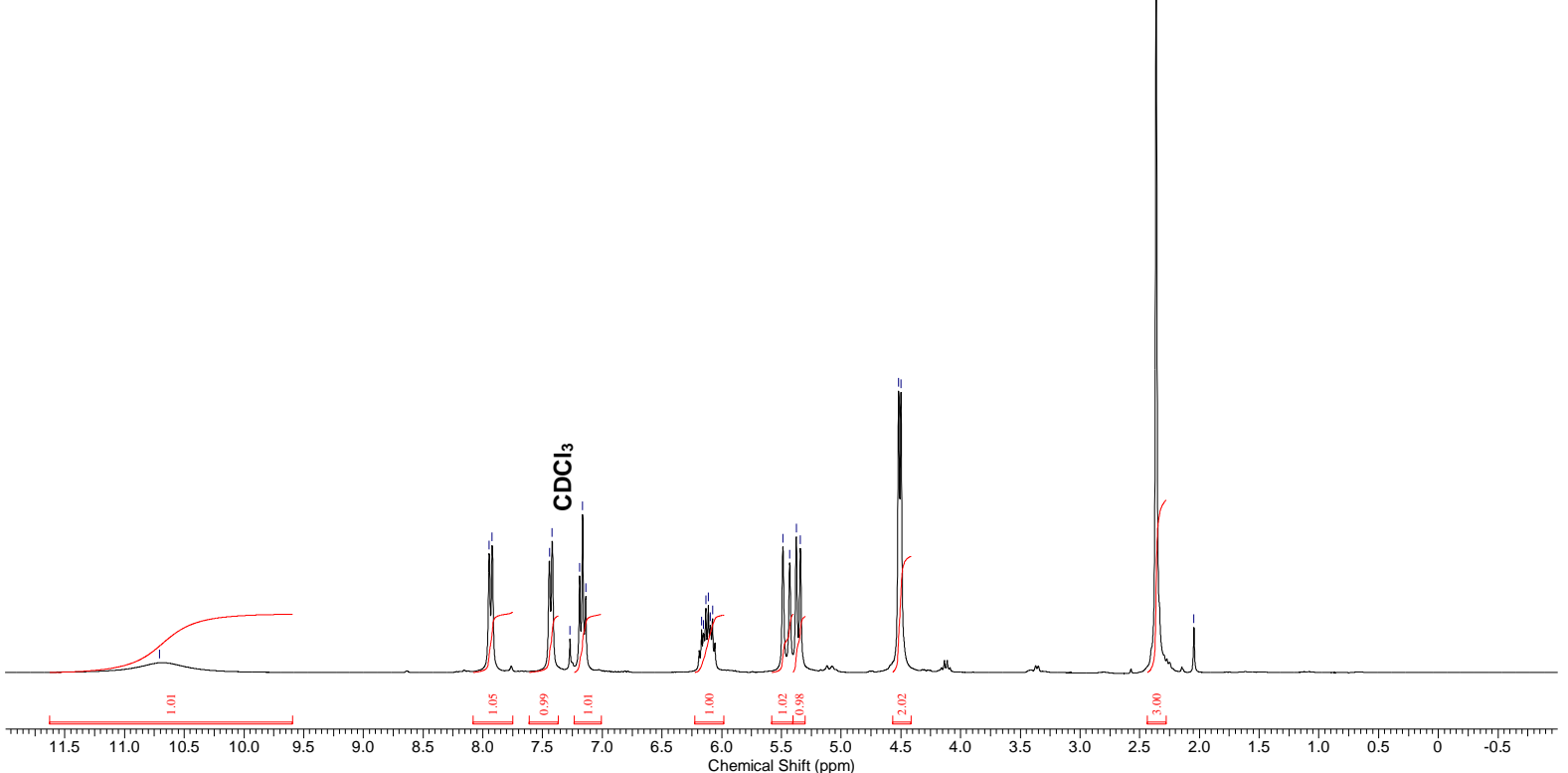

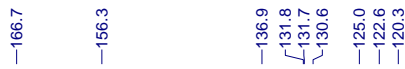

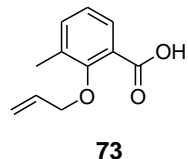

( ${ }^{13} \mathrm{C}$ NMR, $\left.75 \mathrm{MHz}\right)$

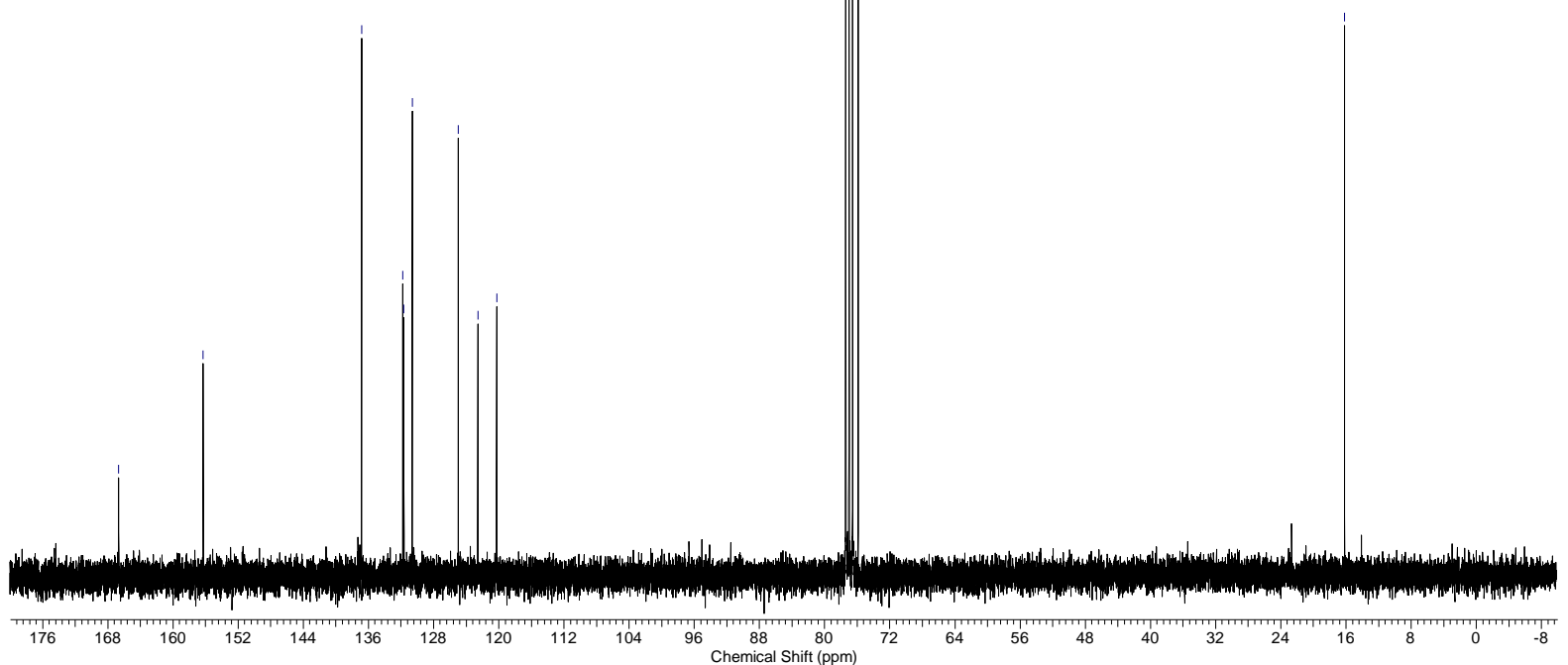




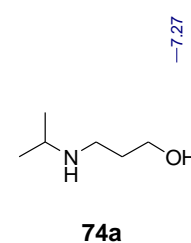

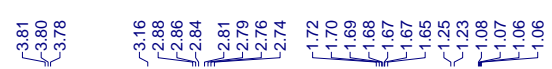

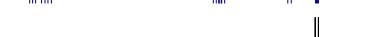

('H NMR, 300 MHz)
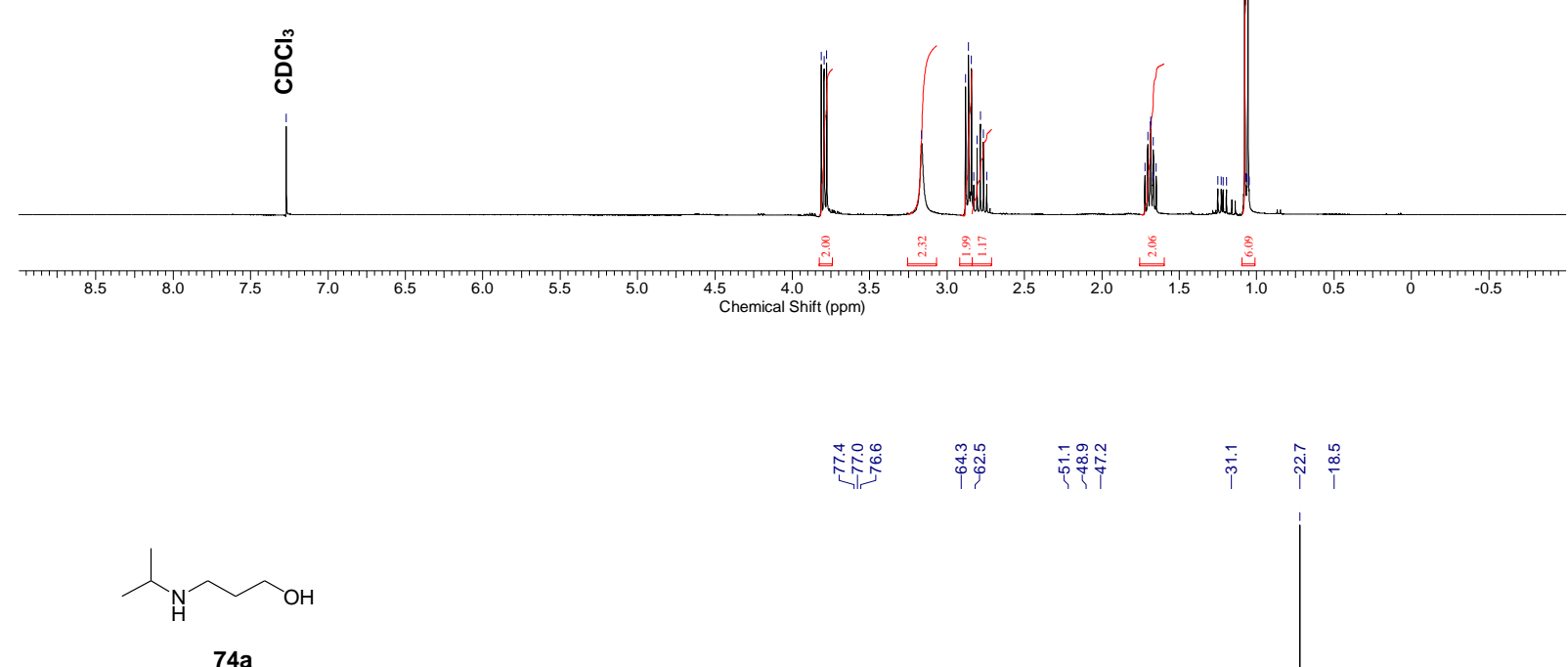

( ${ }^{13} \mathrm{C}$ NMR, $75 \mathrm{MHz}$ )

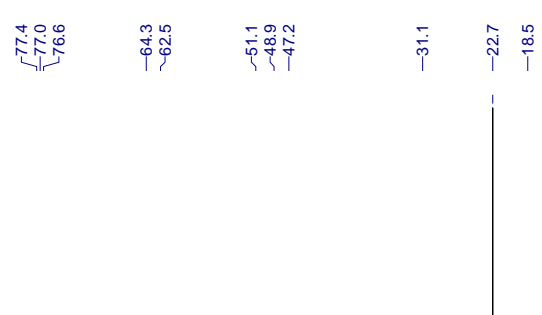

品

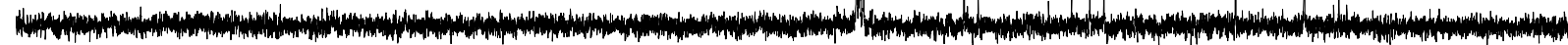

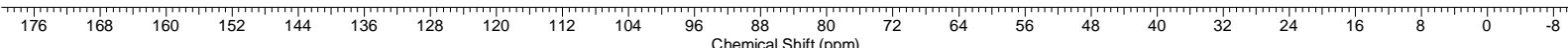




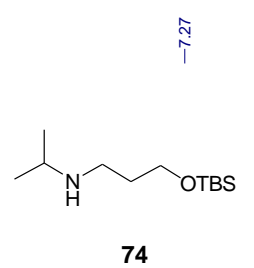

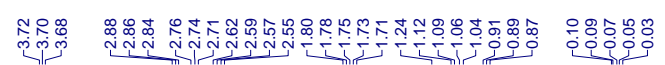

('H NMR, $300 \mathrm{MHz}$ )

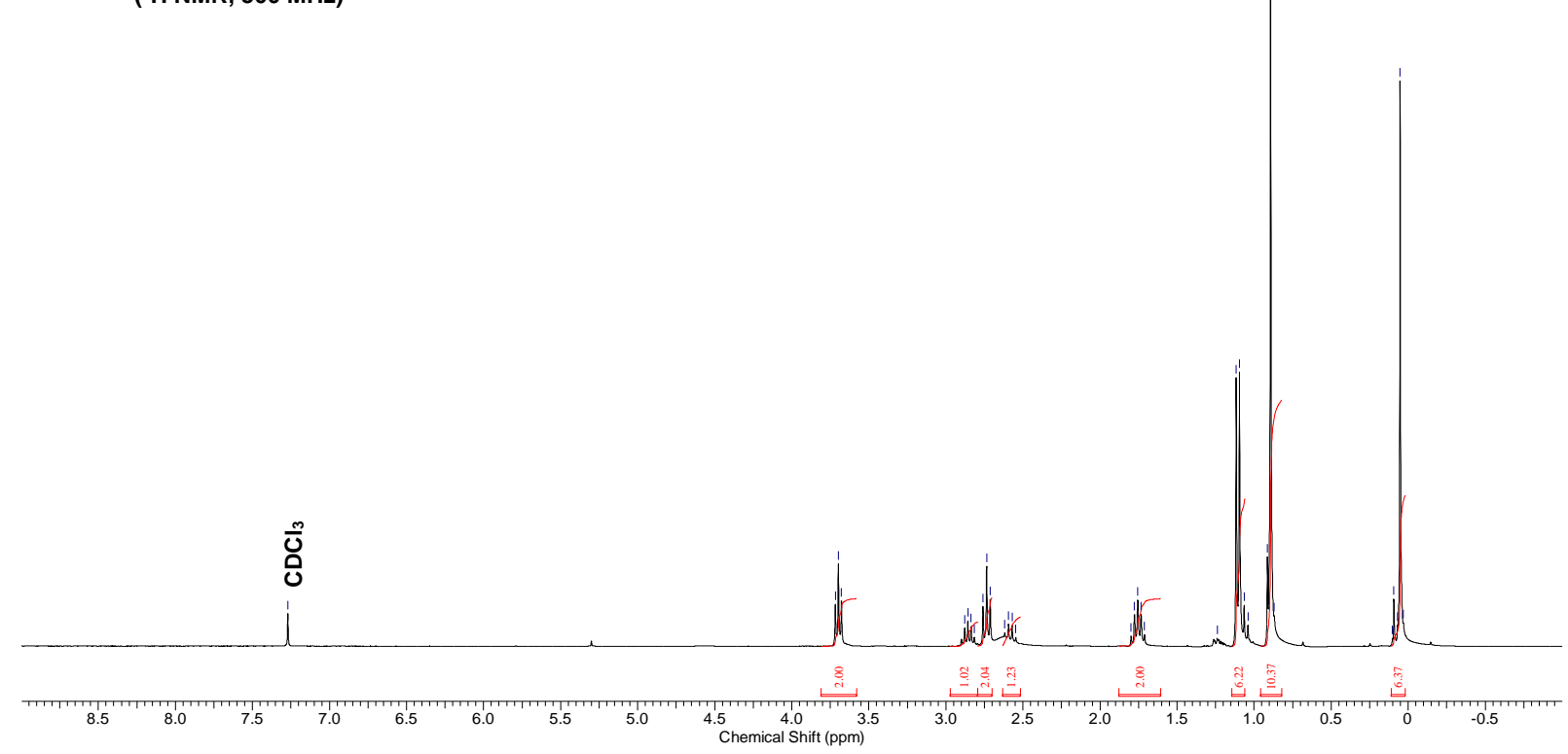

$\lambda_{\mathrm{H}} \sim \sim_{\text {отвS }}$

74

( ${ }^{13} \mathrm{C}$ NMR, $\left.75 \mathrm{MHz}\right)$

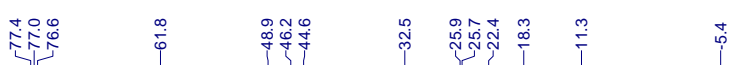

잉

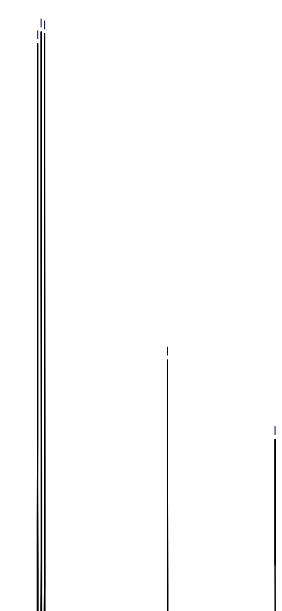




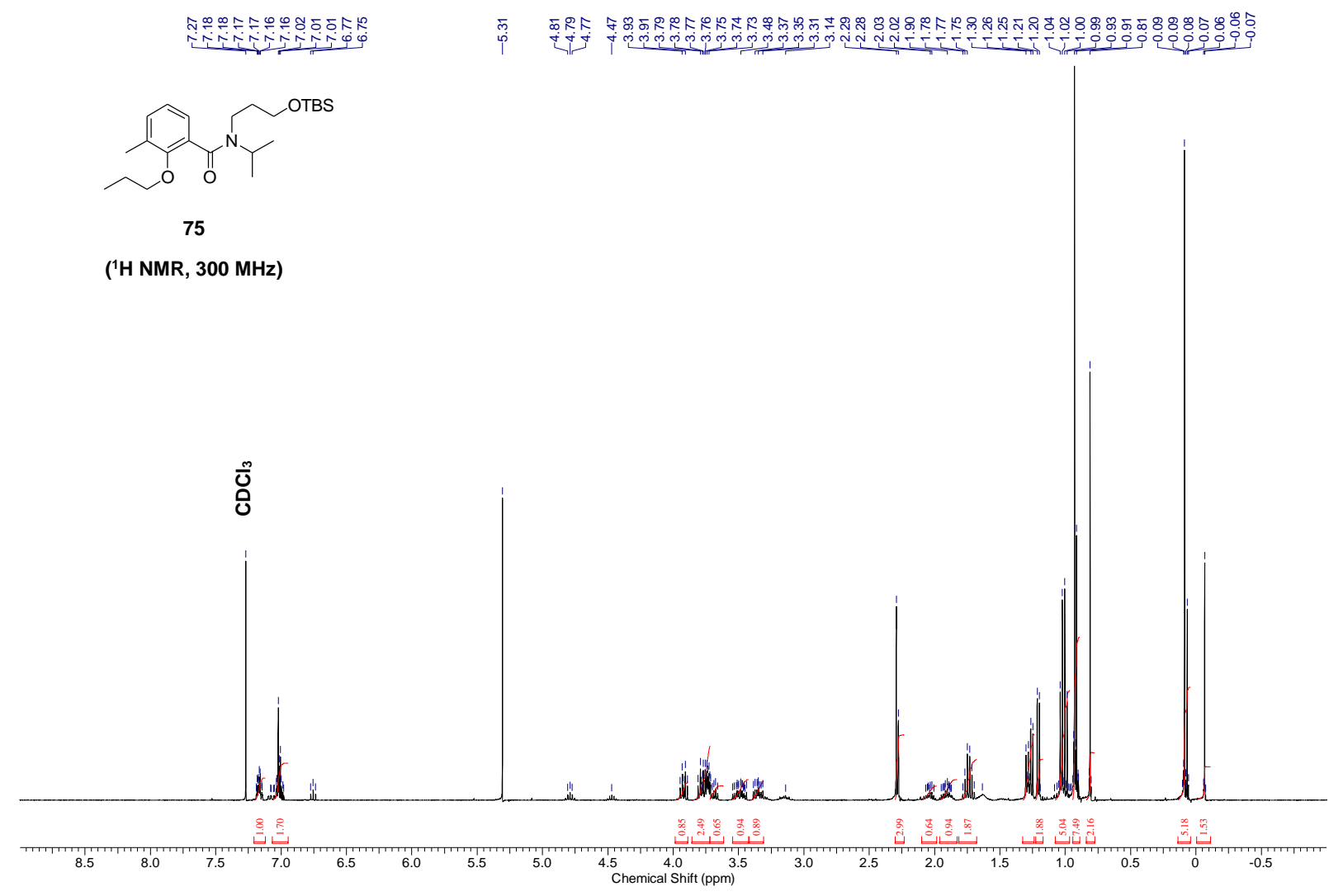

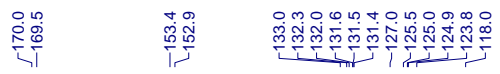

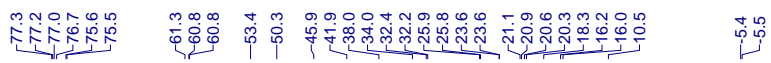

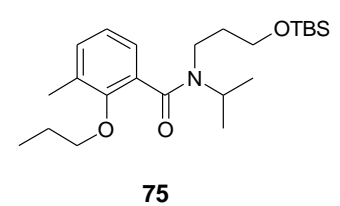

(13C NMR, $75 \mathrm{MHz})$

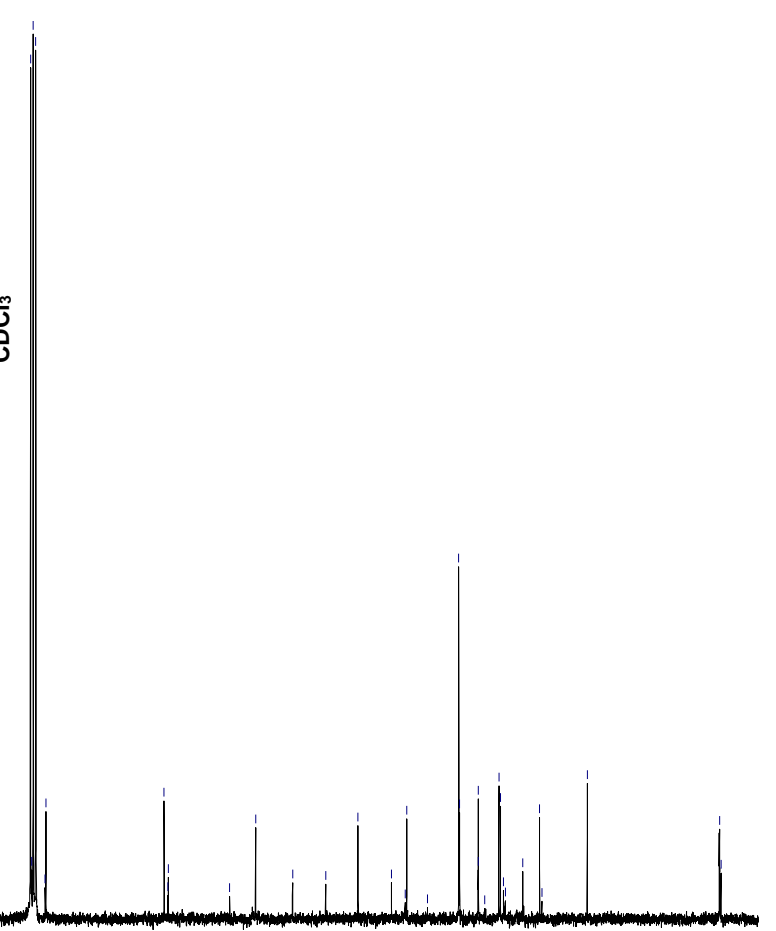

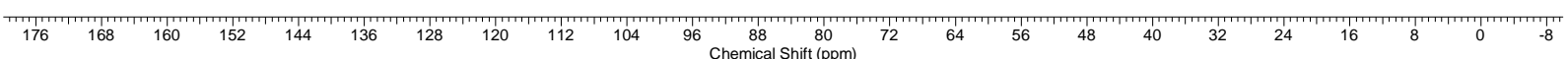




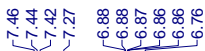

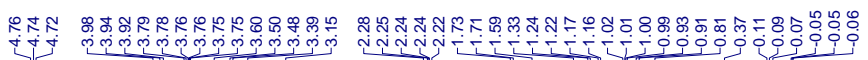

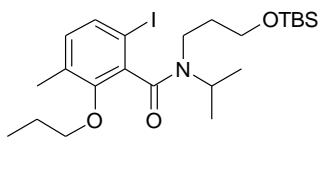

76

('H NMR, $300 \mathrm{MHz}$ )

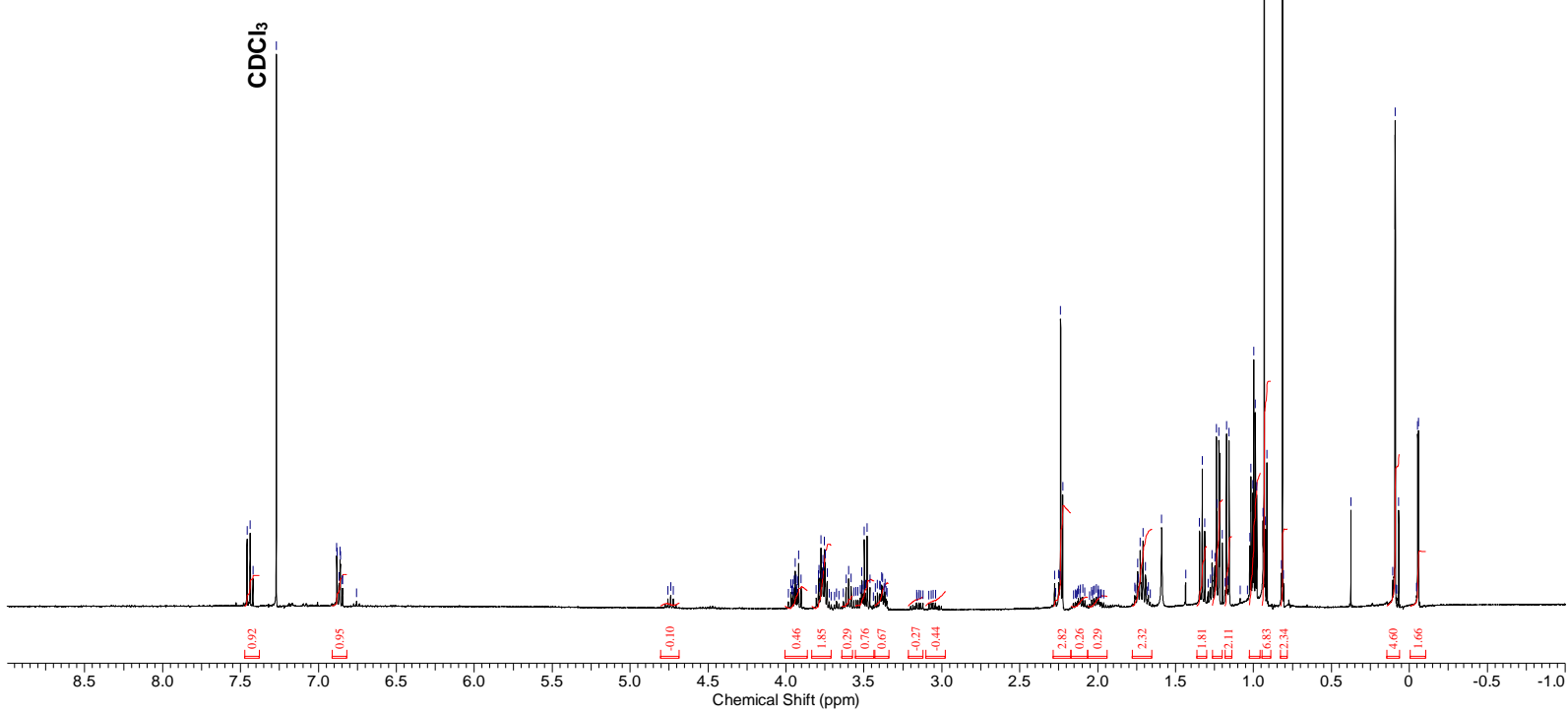

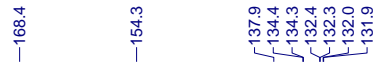

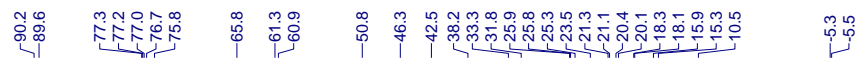

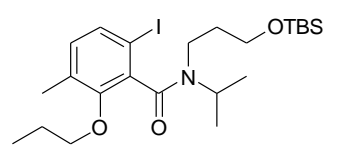

76

( ${ }^{13} \mathrm{C}$ NMR, $75 \mathrm{MHz}$ )

ㅇํㅇ

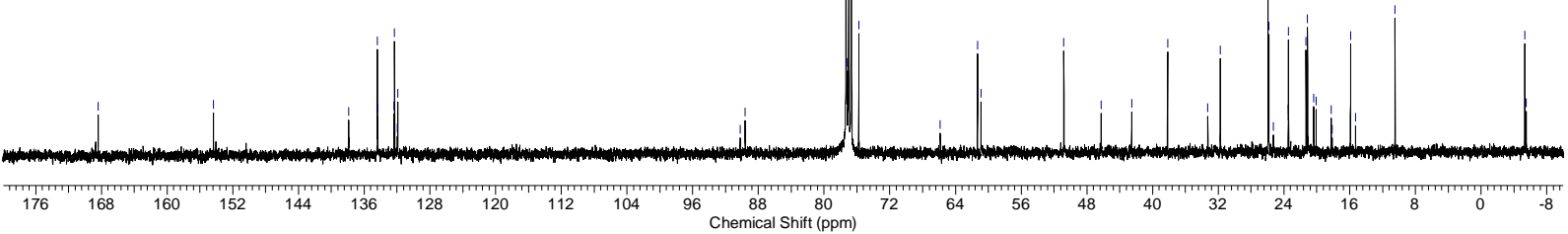




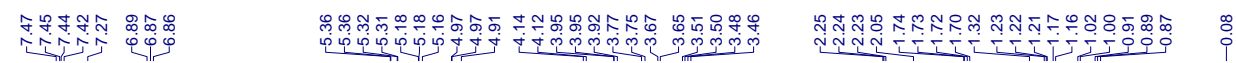

('H NMR, $300 \mathrm{MHz})$

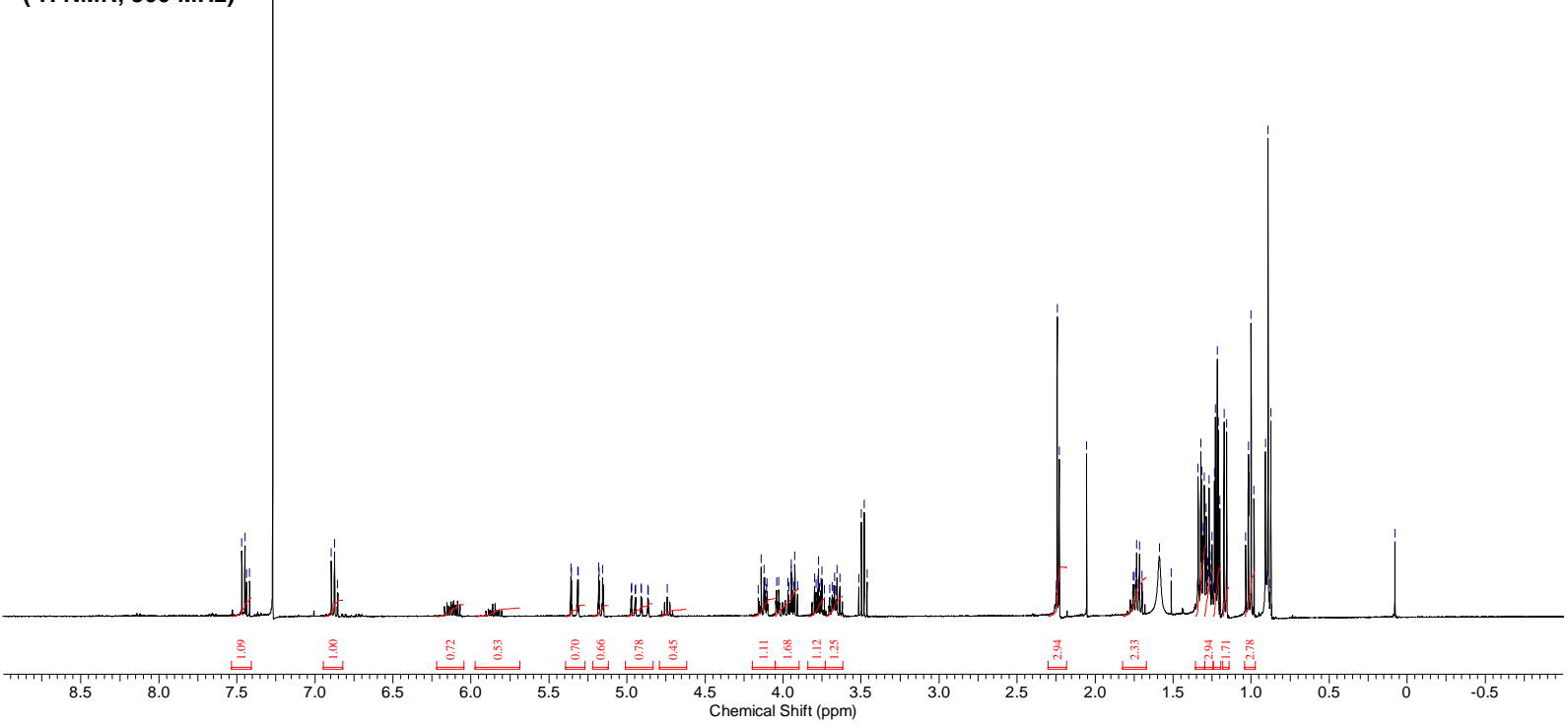

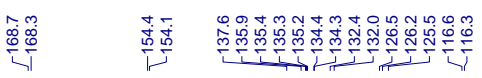

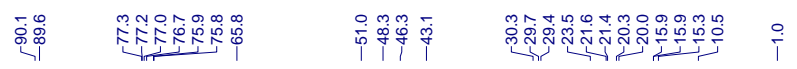

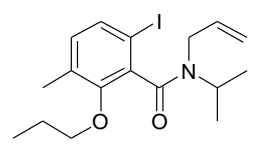

78

( ${ }^{13} \mathrm{C}$ NMR, $\left.75 \mathrm{MHz}\right)$

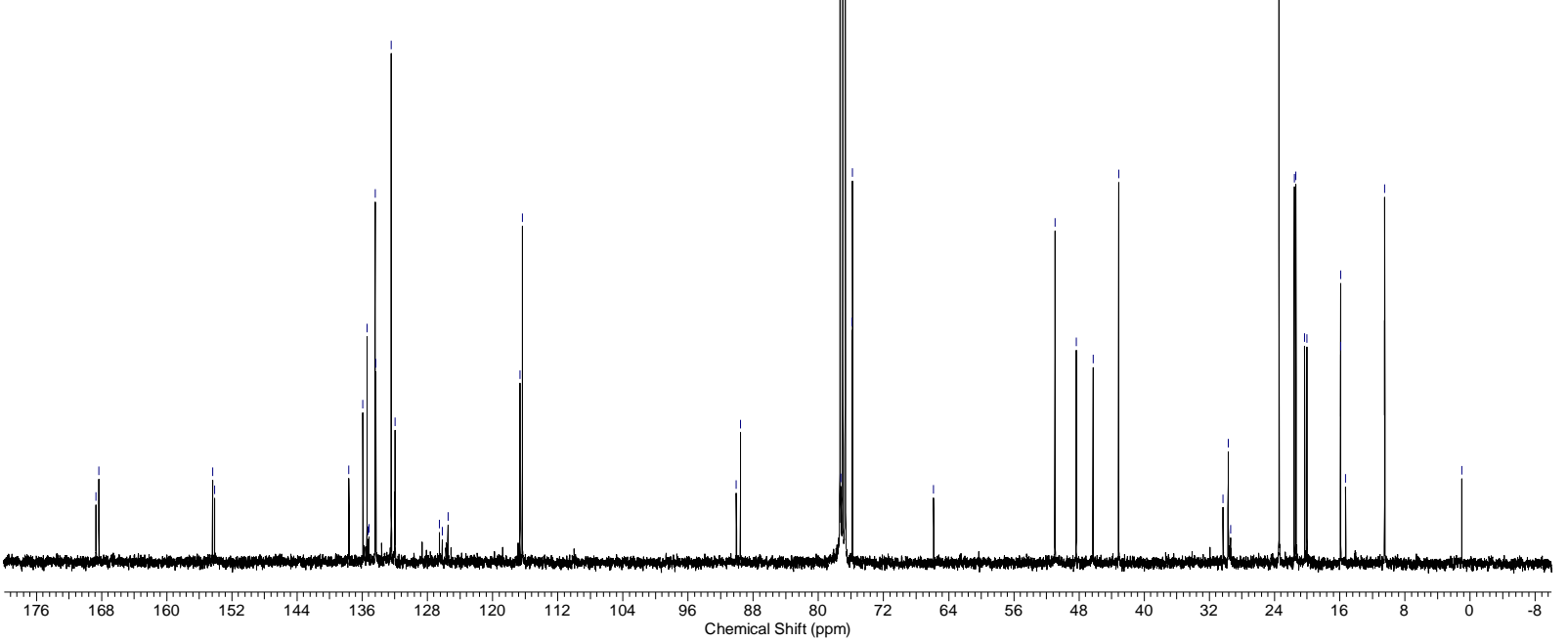

